\title{
A DECOMPOSIÇÃO DA MATRIZ DE PLANEJAMENTO POR VALORES SINGULARES EM MODELOS DE POSTO INCOMPLETO
}

\author{
ANTONIO ASSIZ DE CARVALHO FILHO
}

Orientador: Prof. Dr. ANTONIO FRANCISCO IEMMA

Tese apresentada à Escola Superior de Agricultura "Luiz de Queiroz", da Universidade de São Paulo, para obtenção do título de Doutor em Agronomia, Área de Concentração: Estatística $\theta$ Expe. rimentação Agronômica.

\footnotetext{
PIRACICABA

Estado de São Paulo - Brasil

Novembro - 1987
} 
. i i .

Aos meus pais,

Antonio Assiz e Mari.a Aparecida

e aos meus filhos,

Antonio Assiz de Carvalho Neto e

Afonso Dallapiazza de Carvalho,

OFEREÇO.

A minha esposa Rosangela Maria,

DEDICO. 


\section{AGRADECIMENTOS}

A busca do ideal que aqui se concretiza exigiu cerca de oito anos de luta. Bem ou mal chegamos. No entanto, muitas vezes mãos amigas nos impulsionaram e permitiram que a caminhada näo fosse truncada.

Na verdade, ninguém chega sozinho e seria honesto que esse rol fosse explicitado. Nesse contexto, apesar de erro por omissão, permito-me deixar registrada minha sincera gratidão, dentre muitos para:

Antonio Francisco lemma e Antonio Assiz de Carvalho, os ternos orientadores.

- Humberto de Campos e Décio Barbin, pelo estímulo e apoio em todos os momentos.

- Myrthes da Fonseca Pinto, por suas valiosas recomendacões, amizade e apoio nos bons e nos maus momentos.

- Alvanir de Figueiredo, Carmen D. R. Darë, Josë Fernando Martins Bonilha, Josë Ferrari Lei.te, Marcos Alegre, Maria Kimie Koyanagui Horimoto e Roberto Pannain (in memorian), que confiaram nos primeiros passos. 
- Fernando Ferrari, companheiro de estudo e de luta.

- Maria Helena Ferrete, amiga e incentivadora.

- Maria Helena de Carvalho Guadanhin e José Roberto Guada nhin, pela revisão do manuscrito e preparo do original.

- Suely Asami Takara Mendes Ferreira, pela datilografia da revisão final deste trabalho.

Clarice Garcia Borges Demêtrio, pela redação do SUMMARY.

- Maria tzalina Ferreira Alves e Rosa Maria Alves, pela ami zade, atençãó e apoio dado durante o Curso.

Aos professores do 1. E. Fernando Costa, da gestão do saudo so. diretor Krisan Martin, pelo bom ensino recebido.

- Rosângela Maria, Antonio Assis de Carvalho Neto e Afonso Dallapiazza de Carvalho, que deram sentido a esta vida. 


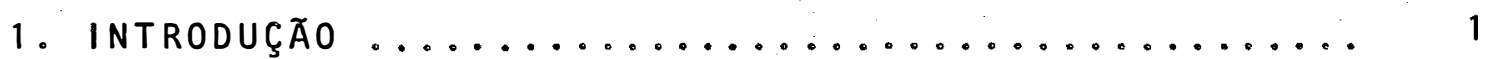

2. REVISÃo de literatura ...................... 4

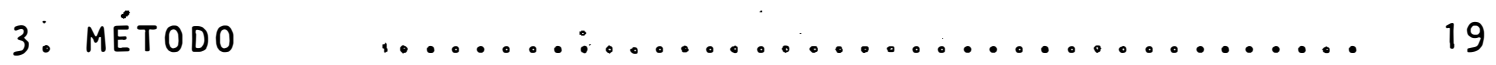

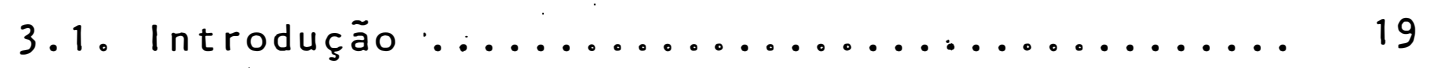

3.2. A Decomposição da Matriz X por Valores Singu-

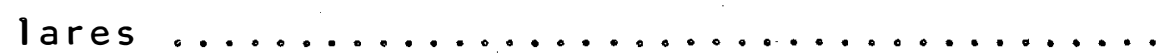

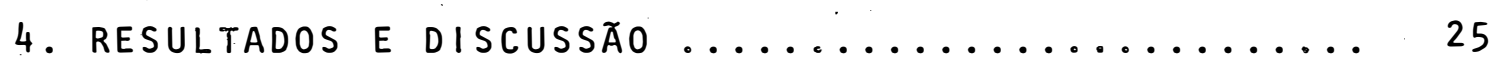

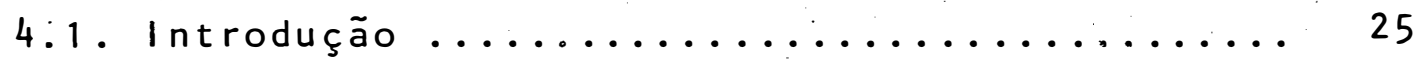

4.2. 0 Modelo Linear de Gauss-Markov ........... 25

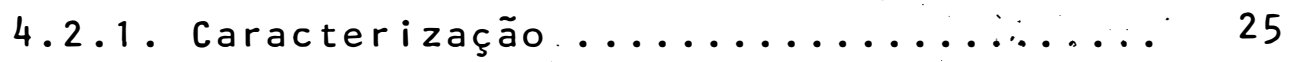

4.2.2. Solução de mínịmos quadrados ........ 26

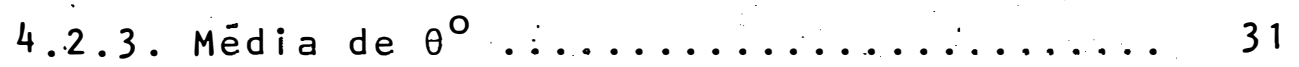

4.2.4. Um estimador não tendencioso para $\sigma^{2} \cdot 32$

4.2.5. Funções estimäveis .............. 33

4.2.6. Obtenção do B.L.U.E. ............ 35

4.3. Sistema de Equações Normais Reduzidas ...... 36

4.3 .1 .0 modelo linear ................. 36

4.3.2. Estimabilidade de um subconjunto de pa râmetros ................... 41

4.3 .3 . Anälise de Variäncia ............. 41 
Pàgina

4.4. Distribuição das Formas Quadräticas ....... 45

4.4 .1$. Introdução ................ 45

4.4.2. A distribuição de y'PU 1 y ........ 46

4.4.3. A distribuição de $y^{\prime}\left(1-P_{U_{1}}\right) y \ldots . . .47$

4.4.4. A distribuição de $y^{\prime}\left(P_{u_{1}}-P_{U_{11}}\right) y \ldots 47$

4.4.5. A distribuição de $y^{i}\left(1-P_{U_{11}}\right) y \ldots 48$

4.5. Intervalos e Regiões de Confiança ......... 48

4.5 .1 Intervalos de confiança .......... 49

4.5 .2 . Regiões de confiança ............ 51

4.5.3. Testes de hipóteses baseados em regiões de confiança .............. 56

4.6. Aplicações dos Resultados Básicos nos Modelos Lineares para um Fator Inteiramente Casuali-

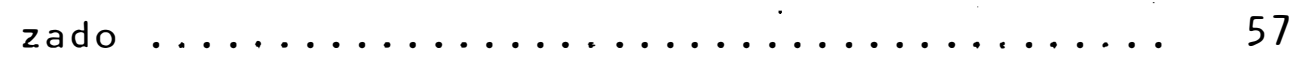

4.6 .1$. Introdução .................. 57

4.6 .2 . 0 algoritmo ................ 60

4.6.3. Alguns resultados üteis ......... 77

4.7. O Modelo Linear com um Fator Inteiramente Cá

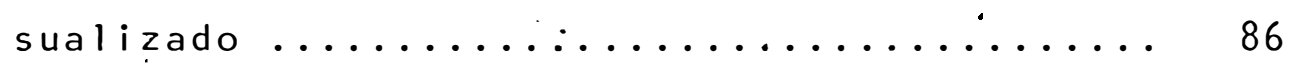

4.7.1. Descrição do modelo ............ 86

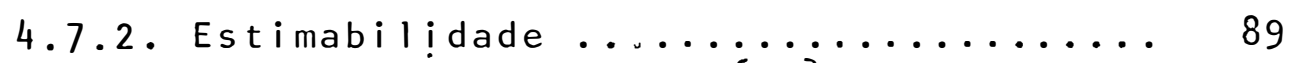

4.7.3. No modelo $Y=\left[x_{2}, Y_{2}\right]\left[\begin{array}{l}\theta_{1} \\ \theta_{2}\end{array}\right]+$. . . . . 91

4.7.4. Distribuição das formas quadráticas .. 99

4.7.5. Estimação por intervalo e por região 101 
.$v i \mathrm{i}$.

Pàgina

4.8. Um Exemplo Numérico.................. 102

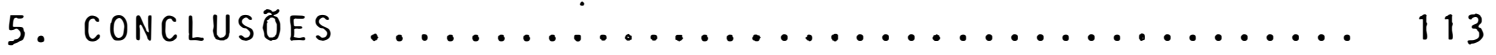

6. REFERENCIAS BIBLIOGRAFICAS ................... 114 


\title{
A DECOMPOSICAO DA MATRIZ DE PLANEJAMENTO \\ POR VALORES SINGULARES EM MODELOS DE POSTO INCOMPLETO
}

\author{
Autor: Antonio Assiz de Carvalho Filho \\ Orientador: Prof, Dr. Antonio Francisco Iemma
}

\section{RESUMO}

Neste trabalho propoe-se uma alternativa para o estudo de modelos lineares através da substituição da matriz do delineamento pela sua Decomposição pelos Valores Sịn gulares (D.V.S).

Visando ilustrar teoricamente o processo, apresenta-se o estudo do modelo linear $y=x \theta+d$ e do modelo linear caracterizado por $y_{\mathbf{i}_{\mathbf{j}}}=\mu+\cdot \mathrm{t}_{\mathbf{i}}+\mathrm{e}_{\mathbf{i}} \mathbf{j}$; com estrutura gaussiana, onde $\mathbf{i}=1,2, \ldots, \mathrm{T}$ tratamentos e $j=1,2, \ldots, R$ repetições.

Verifica-se no desenvolvimento teörico do modelo adotado e na aplicação dos resultados obtidos sobre um exemplo numérico, que a D.V.S. proporciona de modo simples, rápido e objetivo, a obtenção de vários resultados de interesse estatistico, tais como:

- A obtenção de novas regras para verifivar as funções estimáveis no modelo linear $y=x \theta+e$ (G.M.) do presente estudo; 
- 0 estudo das distribuições das formas quadrāticas no modelo reduzido;

- Os testes de interesse estatisticos.

- Além disso, obteve-se um método para decompor a matriz do delineamento ${ }_{n} x_{p}$ pelos Valores Singulares (D.V.S.) sem a utilização de recursos computacionais. 


\title{
THE SINGULAR - VALUE DECOMPOSITION OF THE DESIGN MATRIX IN NOT FULL RANK MODELS
}

\author{
Author: Antonio Assiz de Carvalho Filho \\ Adviser: Prof. Dr. Antonio Francisco Iemma
}

\section{SUMMARY}

An alternative method is proposed for the study of linear models substituting the design matrix its singular-value decomposition (S.V.D.).

The method is illustrated by the study of the linear model $y=x \theta+d$ and the linear $y_{i_{j}}=\mu+t_{i}+e_{i} j_{j}$ with Gaussian structure, where $\mathbf{i}=1,2, \ldots, \mathrm{T}$ treatments and $j=1,2, \ldots, R$ repetitions.

It is showed through the theoretical expansion of the adapted model and the application of the results to a numerical example, that S.V.D. gives in a simple, quick and objective way the following results:

- New rules to verify the estimable functions in the linear model $y=x \theta+e$ under study.

- The study of distributions of the quadratic forms in the reduced model.

The tests of statistical interest. 
.$x i$.

- Besides that a method to decompose the ${ }_{n} x_{p}$ design matrix in its singular values was obtained without the use of computer. 


\section{INTRODUÇÃO}

A Decomposição em Valores Singulares (D.V.S.) tem sido em geral utilizada em modelos lineares de posto com pleto.

Nọ presente estudo, adota-se o modelo linear de Gauss-Markov

$$
n^{Y}=n_{p}^{X} \quad p_{1}^{\ominus}+n^{e} 1
$$

onde

$Y$ é o vetor de observações, $X$ é a matriz de planejamento $(\operatorname{rank}(X)<\min (n, p)), \Theta e ́$ o vetor de parâmetros e e e o erro aleatörio com distribuição normal atribuído à observação $y_{i j}$, tal que $E(e)=\varnothing$ e $\operatorname{Var} .(e)=1 \sigma^{2}$.

Não hä dūvidas de que dada a D.V.S. da matriz de delineamento em modelos 1 ineares de posto incompleto (X), seja possívél a obtenção de vários algoritmos para obter-se diversos resultados estatísticos de interesse. 0 que tem $1 \underline{\mathbf{i}}$ mitado sua utilização ē a dificuldade numérica de obtenção dos autovalores e dos autovetores normalizados dos produtos. matriciais de $X^{\prime}$ e $X^{\prime} X$.

Na anālise de regressão, a substituição da matriz $x$ pela sua D.V.S. tem sido associada à téénica conhe 
cida como Regressão por Componentes Principais, e permite a tingir, de acordo com MANDELL (1982), värios objetivos, entre os quais destaca-se a visualização gräfica de eventuais problemas de colinearidade nas colunas de $x$, e seus efeitos sobre a Anālise de Regressão.

Segundo LIMA FILHO (1981), mesmo que a colinearidade não exista, a D.V.S. fornece os algorítmos mais estäveis para os cálculos relacionados à regressão linear.

0 presente trabalho objetiva:

a) desenvolver alguns aspectos básicos da teoria dos Modelos Lineares de Gauss-Markov (G.M.) pelo enfoque da D.V.S., visto que o assunto é atual e não são comuns as publicações sobre o uso da D.V.S. nos modelos de posto incompleto;

b) aplicar os resultados obtidos no modelo linear de G.M. com um fator inteiramente casualizado, càrac terizado por:

$$
y_{i j}=\mu+t_{i}+e_{i j}
$$

$$
\begin{aligned}
& \text { com } \quad i=1, \ldots, T \text { tratamentos } \\
& \mathbf{j}=1, \ldots, R \text { repet } \mathrm{ições:}
\end{aligned}
$$


c) verificar se a D.V.S. pode ser considerada um bom competidor junto aos procedimentos mais utilizados nesse tipo de delineamento para a solução do sistemä de equa çoes normais e nos testes de interesse, quando o pesquisador não tem o acesso fácil às rotinas computacionais dos värios pacotes estatísticos existentes, tais como o I.M.S.L. (Inter national Mathematical and Statistical Library) e o S.A.S. (Statistical Analisys System), dentre outros. 


\section{REVISÃO DE LITERATURA}

Decompor uma matriz $X$ por seus valores singu lares consiste basicamente em obter uma matriz $S$ formada pelos autovalores de $X X^{\prime}$, uma matriz $U$ dos correspondentes autove tores normalizados de X'X e uma matriz $V$ dos autovetores nor malizados de $X^{\prime} X$, donde se terá:

$$
X=U^{\prime} V^{\prime}
$$

Vários textos, como ACTON (1970), MURDOCH (1970), BAYLEY (1971), COHEN (1973) e STEINBERG (1974) apre sentam definiçōes.e teoremas básicos sobre autovalores e au tovetores de matrizes reais e simétricas.

De acordo com SEARLE (1982), os autovalores de uma matriz $A_{(n)}$ são as raízes de uma equação polinomial em $\lambda$ de ordem $n$, e à medida ém que a dimensãó de $A_{(n)}$ aumen ta, vai se tornando impraticável calcular seu polinômio característico e, consequentemente, encontrar suas raízes. Em muitos casos, conhecendo-se o polinômio característico de uma matriz, a determinação de suas raízes ainda é um proble ma considerado difícil para matrizes de grande dimensão. WILKINSON (1964) descreve värios, algoritmos 
para a obtenção de autovalores e autovetores de matrizes si métricas: vários deles, tais como o Método Periódico de Jaco bi, Givens e de Householder, observa-se que são inspirados no método clässico de Jacobi. No método de Jacobi, a matriz original é posta na forma diagonal atravēs de uma sequência de rotações no plano, usualmente conhecidas como "Transforma ções de Jacobi"ß 0 mētodo de Jacobi não é uma técnica ef ciente para calcular autovalores de matrizes simétricas, pois o nümero de rotações no plano, necessärias para produzir a forma diagonal não é finito, isto é, os elementos fora.da diagonal principal não convergem necessariamente para zero. Na prática, o processo termina quando os elementos fora da diagonal principal são de pequena magnitude, de modo que não comprometam a exatidão dos autovalores obtidos los elementos da diagonal principal).

\section{Sua simplicidade recomenda a sua exposição} nesse trabalho, junto com à observação de que o aperfeiçoa mento dessa técnica forneceu métodos numericamente mais estāveis na redução de matrizes simétricas para matrizes diạ gonais.

Denotando a matriz original por $A_{0}$, descre ve-se, a seguir, o processo de jacobi:

$$
\text { Uma sequência de matrizes } A_{k}, k=0,1 \text {, }
$$

e gerada satisfazendo a relação: 


$$
A_{k}=R_{k} A_{k-1} \cdot R^{\prime}{ }_{k}
$$

onde a matriz $R_{k}$ é determinada como se segue:

$$
\text { Suponha-se que os elementos fora da diagonal }
$$

principal de $A_{k-1}$ de maior valor absoluto esteja na posição $(p, q)$ (p-ēsima linha, q-ésima coluna), então $R_{k}$ corresponde à rotação no plano $(p, q)$ e o ângulo o da rotação é selecionado de modo que reduzao elemento em $(p, q)$ de $A_{k-1}$ para ze ro. Tem-se então desde que $\mathrm{p}<\mathrm{q}$

e

$$
\begin{aligned}
& R_{p p}=R_{q q}=\cos \theta \\
& R_{p q}=-R_{q p}=\operatorname{sen} \theta
\end{aligned}
$$

$$
R_{i j}=\left\{\begin{array}{lll}
1(i, j \neq p, q) \\
0 \text { caso contrário }
\end{array}\right.
$$

$A_{k}$ difere de $A_{k-1}$ somente na p-ésima linha e na q-ésima coluna. Todas $A_{k}(k=0,1, \ldots)$ são simétricas. Define-se os valores modificados da seguinte maneira:

$$
\begin{aligned}
& a_{i, p}^{(k)}=\underset{i, p}{a^{(k-1)}} \cos \theta+a_{i, q}^{(k-1)} \operatorname{sen} \theta=a^{(k)} \\
& a_{i, q}^{(k)}=-a_{i, p}^{(k-1)} \operatorname{sen} \theta+a_{i, q}^{(k-1)} \cos \theta=\underset{q}{(k)}
\end{aligned}
$$




$$
\begin{aligned}
& a^{(k)}=a^{(k-1)} \cos ^{2} \theta+2 a^{(k-1)} \cos \theta \operatorname{sen} \theta+a^{(k-1)} \operatorname{sen}^{2} \theta \\
& p, p \quad p, p \quad p, q \quad q, q \\
& \underset{q, q}{a_{p, p}^{(k)}}=\underset{p, q}{a(k-1)} \operatorname{sen}^{2} \theta-2 \underset{q, q}{(k-1)} \cos \theta \operatorname{sen} \theta+a a^{(k-1)} \cos ^{2} \theta
\end{aligned}
$$

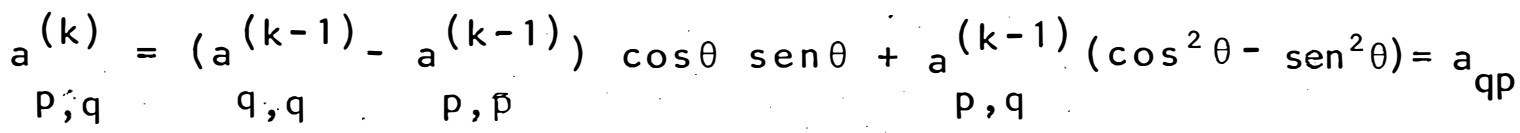$$
\text { De acordo com WILKINSON (1964), sendo }
$$

$$
a_{p, q}^{(k)}=0
$$

$\theta$ é definido da seguinte maneira:

$$
\left.\tan 2 \theta=2 \underset{q, p}{a^{(k-1)}} / \operatorname{pp}_{p p}^{(k-1)}-a_{q q}^{(k-1)}\right)
$$

donde

$$
\theta=\frac{1}{2} \operatorname{arctg}\left[\frac{2 a \frac{(k-1)}{q, p}}{a_{p, p}^{(k-1)}-a_{q, q}^{(k-1)}}\right] \text {. }
$$

se $a_{p, p}^{(k-1)}=a_{q, q}^{(k-1)}$, toma-se $\theta=+\pi / 4$, concordando com o si nal de $a^{(k-1)}$. qp

0 processo. e essencialmente iterátivo, uma 
vez que um elemento qué foi reduzido à zero por uma rotação é, em geral, diferente de zero nas rotaçōes subsequentes.

WILKINSON (1964) prova que se $\theta$ é tomado do intervalo $|\theta| \leqslant \pi / 4$, então

$$
A_{k} \longrightarrow \text { diag }\left(\lambda_{i}\right) \text { quando } k \rightarrow \infty
$$

onde os $\lambda_{i}$ são os autovalores de $A_{0}$, e consequentemente para todos $A_{k}$.

Uma estratēgia simples de seleção dos elemen tos para efetuar-se as rotações no plano, obtém-se tomando-se os elementos da matriz A na ordem: $(1,2),(1,3), \ldots$, $(1, n) ;(2,3),(2,4), \ldots,(2, n) \ldots,(n-1, n)$, e então retorna-se para o elemento $(1,2) \quad$ Tal esquema é denominado mé todo de Jacobi Periōdico.

HENRICI (1958) demonstra que o método de Jacobi Periódico realmente converge, se os ângulos de rotação são $1 \mathrm{imitados}$ adequadamente $|\theta| \leqslant \pi / 4$.

A transformação de Jacobi não é um método prä - tico para reduzir matrizes simétricas para matrizes diagonais, por não permitir construir um método eficiente tal que não destrụa os zerọs já obtidos anteriormente.

De acordo com IEMMA $(1982)$, dada $A(k)$ real e uniforme, existe uma matriz de Helmert $H_{(k)}$, tal que $H A H^{\prime}=D$, 
oncie D é a matriz.diagonal que espelha os autovaloresde A e $H$ e uma matriz ortogonal cujas linhas contêm os autovetores normalizados de A.

Além disso, a matriz $H_{(k)}$ tem lei de formaçao extremamente simples, dependente apenas de: sua dimensão K.

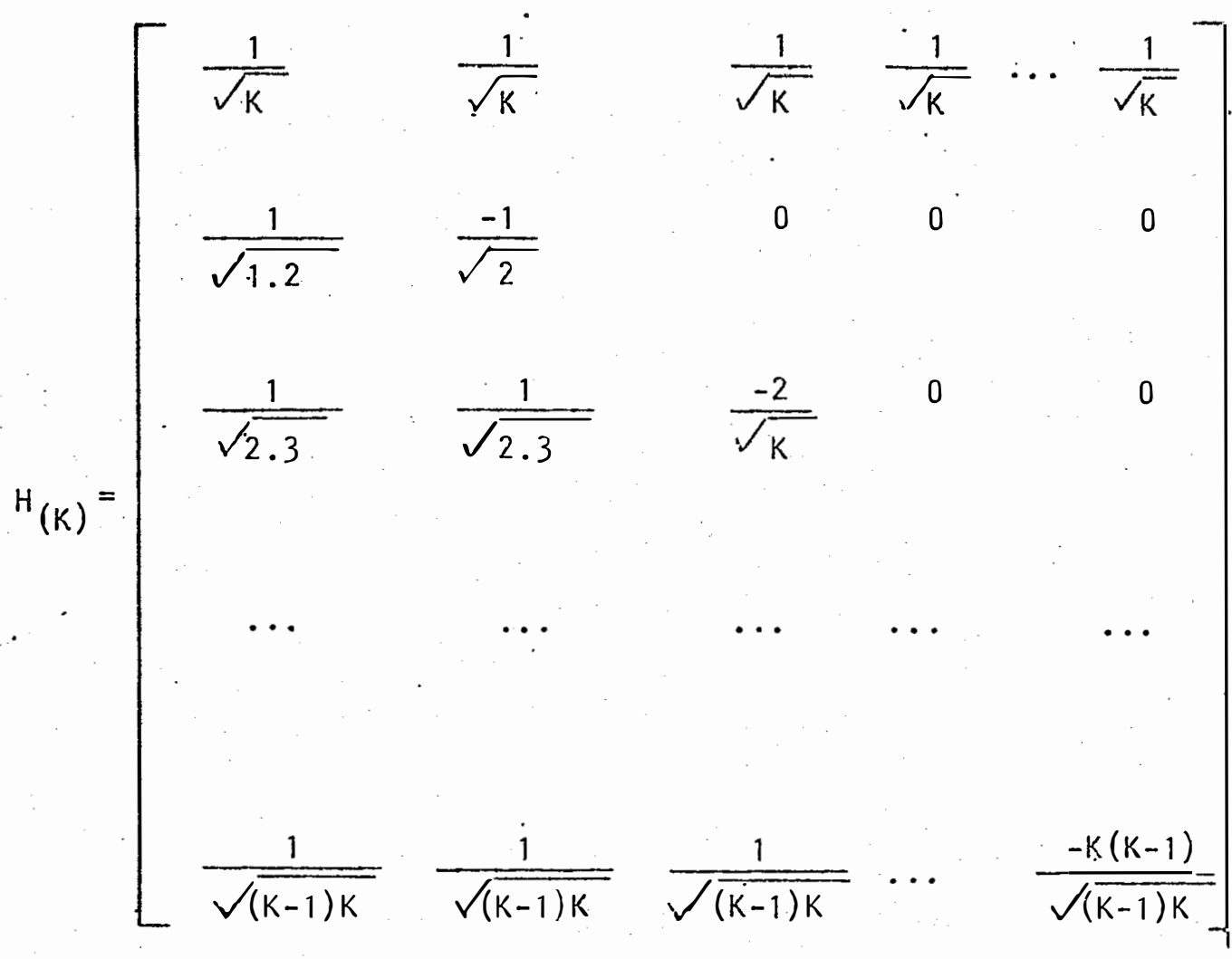

IEMMA (1985), fazendo $H_{(k)}=\left[\begin{array}{l}h ! \\ \hdashline \\ H^{*}\end{array}\right]$ obtém a decomposição espectral de $A_{(k)}$ real e uniforme atravēs de

$$
A=\gamma_{1} h^{\prime}+\gamma_{2} H^{*} H^{*}
$$


onde $\gamma_{1}$ e $\gamma_{2}$ são autovalores de $A, \gamma_{2}$ com multiplicidade K-1. De modo anālogo, obtém a inversa clássica generalizada de Moore - Penrose para A de posto incompleto, respectivamente por:

$$
\begin{aligned}
& A^{-1}=\frac{1}{\gamma_{1}} h h^{\prime}+\frac{1}{\gamma_{2}} H^{*} H^{*} \\
& A^{+}=\frac{1}{\gamma_{2}} H^{*} H^{*}
\end{aligned}
$$

Mostra também que uma condição necessāria e suficiente para que as soluções de mínimos quadrados ordinärios sejam solu ções das equạções de Aitken é que $P$ e $\Omega$ comutem. Aqui. $P$ é projetor ortogonal de $y$ em $C(x)$ e $\Omega$ é a matriz de variâncias e covariâncias.

IEMMA (1987a) apresenta procedimentos gerais para a manipulação didática de conceitos como Decomposição Espectral, Decomposição por Valores Singulares e define a matriz $k^{n}$ - uniforme, obtida pelo produto de Kronecker entre $n$ matrizes uniformes, apresentando as regras básicas pạ ra a obtenção de seus autovalores.

- Sejam $A_{1}, A_{2}, \ldots, A_{n}$ matrizes uniformes e se.jam $H_{1}, H_{2}, \ldots, H_{n}$ matrizes de Helmert tais que $H_{i} A_{i} H_{i}^{\prime}=D_{i}$. Então,

$$
\begin{aligned}
& \left(H_{1} \otimes H_{2} \otimes \ldots\left(\otimes H_{n}\right)\left(A_{1} \otimes A_{2} \otimes \ldots \otimes A_{n}\right)\left(H_{1}{ }^{\prime} \otimes H_{2}^{\prime} \otimes \ldots \otimes H_{n}^{\prime}\right)=\right. \\
= & D_{1} \otimes D_{2} \otimes \ldots \otimes D_{n}=D=\text { matriz diagonal que exibe os autova- }
\end{aligned}
$$


lores da matriz $B=K^{n} A_{j}$.

os três ültimos trabalhos citados irão alicer çar o estudo da obtenção da D.V.S. da matriz ${ }_{n} X_{p}$ no. linear do presente estudo.

Por outro lado, no modelo linear ${ }_{n} \gamma_{1}=n^{X}{ }_{p} \cdot p^{\theta_{1}}+n^{e}{ }_{1}$, o posto da matriz ${ }_{n} x_{p}$ é, em geral, nos delineamentos experimentais, menor que o minimo entre $p$ e $n$, tendo então ${ }_{n} X_{p}$ posto coluna incompleto, e consequentemente o siste ma de equações normais (S.E.N.) $x^{\prime} x \hat{\theta}=x^{\prime} y$ sempre consisten te, è nesse caso, indeterminado.

Para solução do sistema dẹ equações normais indeterminado, encontra-se, entre outros, o procedimento de inversas generalizadas.

Ụma solução do sistema de equações normais a través da inversa generalizada pode ser obtida, por exemplo, em RAO (1965), SEARLE (1971), SEBER (1977), SEARLE (1984) e IEMMA (1985) por $\hat{\theta}=\left(X^{\prime} X\right)^{+} X^{\prime} y$, onde $\left(X^{\prime} X\right)^{+}$é a inversa generalizada de Moore-Penrose de $X^{\prime} X$.

- De acordo com GRAYBILL (1969), para cada matriz $x^{\prime} X$, de posto $t$, existe uma ünica matriz $\left(x^{\prime} X\right)^{+}$e ela é definida por

$$
\left(X^{\prime} X\right)^{+}=C^{\prime}\left(C C^{\prime}\right)^{-1}\left(B^{\prime} B\right)^{-1} B^{\prime}
$$

onde ${ }_{p} X^{\prime} X_{p}=p^{B}{ }_{t}{ }^{C}{ }_{p}$ e $r\left(X^{\prime} X\right)=r(B)=r(C)$. 
Sem dūvida, a obtenção de $\left(x^{\prime} x\right)^{+}$é, em geral, bastante trabalhosa, mormente quando não se pode contar com o uso de pacotes estatísticos para computação. No entanto, a obtenção de B e C pode ser com clareza simplificada através do uso do excelente algoritmo de DWIVEDI (1975).

SEBER (1977) utiliza o procedimento de redú zir o modelo ao de posto coluna completo, onde considera-se apenas. as colunas linearmente independentes de $X$. Chamando- se essa matriz de $X_{1}$, a solução do sistema e dada por $\hat{\theta}=\left(x_{1}^{\prime} x_{1}\right)^{-1} x_{1}^{\prime} y$.

CARVALHO (1982) considera o modelo linear $y=$ $X \theta+e$ com as condições G-M em e, e toma $X=U_{1} S_{1} V_{1}$ a decom posição em valores singulares de $X$. O valor de $\theta$ que minimiza a nor ma Euclidiana ||$y-x \theta||$ é dada por

$$
\hat{\theta}=v_{1} s_{1}^{-1} U_{1}^{\prime} y
$$

onde a obtenção de $V_{1} S_{1}^{-1} U_{I}^{\prime}$ é, em geral, feita com auxílio de pacotes estatísticos.

0 uso de restrições não estimäveis nas soluções tem sido, segundo RAO (1965), SEARLE (1971) e SEBER (1977), o mais empregado dentre os procedimentos de buscade solução única para o sistema de equações normais.

GOMES (1967) propõe que a restrição, $M \theta^{0}=\phi$ Seja imposta a matriz $X^{\prime} X$, obtendo-se uma matriz (X'X- $M$ ) não singular, facilitando a obtenção de uma forma geral pa- 
ra as soluções do S.E.N. restrito:

SEARLE (1971) distingue restrição paramētrí ca e restrições nas soluções (contraint). As restrições pạ ramētricas são originadas da definição feita sobre os parâmetros. Como exemplo considera os parâmetros como os trés angulos de um triângulo. A restrição natural é que a sua so ma seja $180^{\circ}$. Já a restrição nas soluções tem como objetivo determinar solução única para o S.E.N., evitando o uso de inversas generalizadas.

$$
\text { RAO (1965) e CARVALHO (1982) não fazem essa }
$$

dístinção, e determinam o S.E.N. com restrição paramétrica não estimāvel através da minimização de ||$y-x \theta||^{2}$ sujeito à restrição $B^{\prime} \theta=C$ pelo método dos multipliçadores de Lagrange, obtendo:

$$
\left[\begin{array}{c:c}
X^{\prime} & X^{\prime} \\
\hdashline B^{\prime} & \theta
\end{array}\right]\left[\begin{array}{c}
\theta^{0} \\
\hdashline \ell
\end{array}\right]=\left[\begin{array}{c}
x^{\prime} y \\
\hdashline c
\end{array}\right]
$$

onde $\ell$ e um vetor de multiplicadores de Lagrange.

SEARLE (1984) considera uma inversa general $\underline{\mathbf{i}}$ zada $\left(X^{\prime} X\right)^{+}=G$, da qual obtēm a inversa generalizada $G_{r}$ s jeita a restrição não estimävel $\dot{B}^{\prime} \dot{\theta}=\emptyset$, sendo que $B$ tem pos to coluna completo.

Geralmente utiliza-se desse processo quando se tem acesso fácil a rotinas computacionais como o S.A.S.G.L.M. dentre outros, obtendo-se úm vetor solução (com al- 
guns elementos iguais a zero) e G, que são convertidos para o modelo com as restrições impostas; resultando:

$$
\begin{aligned}
G_{r} & =G-(I-H)\left[B^{\prime}[1-H]\right]^{+} B^{\prime} G, \text { onde } \\
H & =\left(X^{\prime} X\right)^{+} X^{\prime} X \text { e } \theta_{r}^{0}=G_{r} X^{\prime} y
\end{aligned}
$$

De acordo com GRAYBILL (1961) e JOHN (1971), outro procedimento utilizado na busca do modelo de posto completo tem sido a reparametrização, a qual consiste em di minuir o nümero de parâmetros através de transformações lineares, de tal forma que a matriz $x$ obtida seja de rank coIuna completo.

CARVALHO (1982) apresenta uma condição para que dois modelos sejam reparametrizações um do outro.

Nos modelos. lineares do presente estudo, fre quentemente surge o interesse sobre funções lineares dos pa râmetros; entretanto, nem todas as funções lineares dos parâmetros são identificáveis dos dados.

De acordo com CARVALHO (1982), no modelo $y=x \theta+d$, a função linear paramétrica $\lambda^{\prime} \theta$ é identificável se - e sō se $\lambda^{\prime} \varepsilon R(X)$, onde $R(X)$ denota o espaço gerado pelas linhas de $X$; e destaca a diferença entre os conceitos de i dentificabilidade (no modelo linear sem estrutura de erros) e estimabilidade (no modelo estocāstico). da função $\lambda^{\prime} \theta$. Segundo RAO (1945), o melhor estimador line- 
ar não viezado (b.l.u.e.) da função-estimável $\lambda ı \theta$ é dado por $\widehat{\lambda^{\prime} \theta}=\lambda^{\prime} \theta^{0}$ (Teorema de Gauss-Markov), onde $\theta^{\mathrm{O}}$ é qual quer solução das equações normais. Naturalmente $\theta^{0}=x^{+} y$ e $\hat{\theta}=V_{1} S_{1}^{-1} U_{1}^{1}$ y são elementos do conjunto de soluções.

IEMMA (1987b), utilizando os conceitos de De composição Espectral, de D.V.S. e de Equações Normais Reduzidas, apresenta a lei de formação para as matrizes inver sas generalizadas de Moore-Penrose nos delineamentos em Blo cos Casualizados, Blocos Incompletos Balanceados, Redes e Quadrados Latinos. Através das leis de formação, verifica que o sistema de E.N.R. CT $=$ Q pode ser resolvido sem a in veŕsão da matriz C. Assim, uma solução géral pode ser obtida no modelo irrestrito por $\hat{\theta}^{\circ}=m C Q$, onde $m e^{-}$um valor fa cilmente determinável e constante para cada planejamento.

$$
\text { Apresentaremos a seguir alguns resultacios; }
$$
já conhecidos da literatura atinente a modelos lineares, em face aos objetivos desse estudo.

Definição 1 - Dada a matriz ${ }_{m}^{A}{ }_{n}$, se existe a matriz ${ }_{n}{ }^{+}{ }_{m}$ que satisfaz às quatro condições a seguir, então ${ }_{n} A_{m}^{+} e$ de finida como a inversa de Hoore-renrose de A. 

i) $\mathrm{AA}^{+}$é simétrica
i i i) $\mathrm{A}^{+} \mathrm{AA}^{+}=\mathrm{A}^{+}$
ii) $A^{+} A$ é simétrica
iv) $\mathrm{AA}^{+} \mathrm{A}=\mathrm{A}$

Teorema 1 (GRAYBILL, 1969)

Para cada matriz $m^{A}$, de posto $r$, existe uma única matriz ${ }_{n} A_{m}^{+}$e ela é definida por:

$$
\begin{gathered}
A^{+}=C^{\prime}\left(C C^{\prime}\right)^{-1}\left(B^{\prime} B\right)^{-1} B^{\prime}, \quad \text { onde } \\
\dot{m}^{A}{ }_{n}=m^{B} r r_{n}^{C} \quad \text { e } \quad r(A)=r(B)=r(C)=r
\end{gathered}
$$

Teorema 2 (GRAYBILL, 1969)

Dada as matrizes ${ }_{m}^{A}{ }_{n}$ e ${ }_{n}{ }^{M}$, então:
i) $\left(A^{+}\right)^{\prime}=\left(A^{\prime}\right)^{+}$
i i) $\left(A^{+}\right)^{+}=A$
iii) $\left(A^{\prime} A\right)^{+}=A^{+} A^{+}$
iv) $\left(A A^{+}\right)^{+}=A A^{+}$e $\left(A^{+} A\right)^{+}=A^{+} A$
v) $A^{2}=A \Leftrightarrow A A^{+}=A^{+} A$
vi) Se $r(A)=m$, então $A^{+}=A^{\prime}\left(A A^{\prime}\right)^{-1}$ e $A A^{+}=$i
Se $r(A)=n$, então $A^{+}=\left(A^{\prime} A\right)^{-1} A^{\prime}$ e $A^{+} A=$,
vii) Se $\exists A^{-1} \Rightarrow A^{-1}=A^{+}$ 
viii) Se $A$ é idempotente, então $A^{+}=A$

$$
\begin{aligned}
& \text { ix) Se } r(A)=r(M)=n \text {, entäo }(A M)^{+}=M^{+} A^{+} \\
& \text {x) } r\left(A^{+}\right)=r\left(A A^{+}\right)=r\left(A^{+} A\right)=r\left(A A^{+} A\right)=r\left(A^{+} A A^{+}\right)
\end{aligned}
$$

Teorema 3 (GRAYBILL, 1969)

$$
\text { Seja } m^{A} \text { uma matriz que transforma o vetor }
$$
$y \in V_{n}$ no vetor $\hat{y} \varepsilon C(A)$, isto é, $\bar{y}={ }_{m}^{A}{ }_{n} n^{\theta}$, então existe uma matriz simétrica e idempotente

$$
P=A A^{+}
$$

tal que $y=$ Py. Nesse caso, $P$ é projetor ortogonal do vetor y sobre o espaço coluna de $A$.

Teorema 4 (GRAYBILL, 1961)

0 vetor $\theta^{0} e^{-}$solução de mínimos quadrados de $x \theta-y=e(\theta)$, se e somente se o vetor $x \theta^{0}$ é projeção ortogonal de $y$ no espaço coluna de $x$. Nesse caso, $\hat{y}=x \theta^{\circ}=$ $P_{y}$ é a aproximação de mínimos quadrados para $y$.

Os resultados enunciados a seguir, podem ser encontrados em SEARLE (1971, cap. 5), dentre outros. Se jam :

${ }_{n} y_{1}$ um vetor de variáveis aleatórias com distribuição nor mal tal que $y \sim N\left(\mu, \Omega \sigma^{2}\right)$; 
.18.

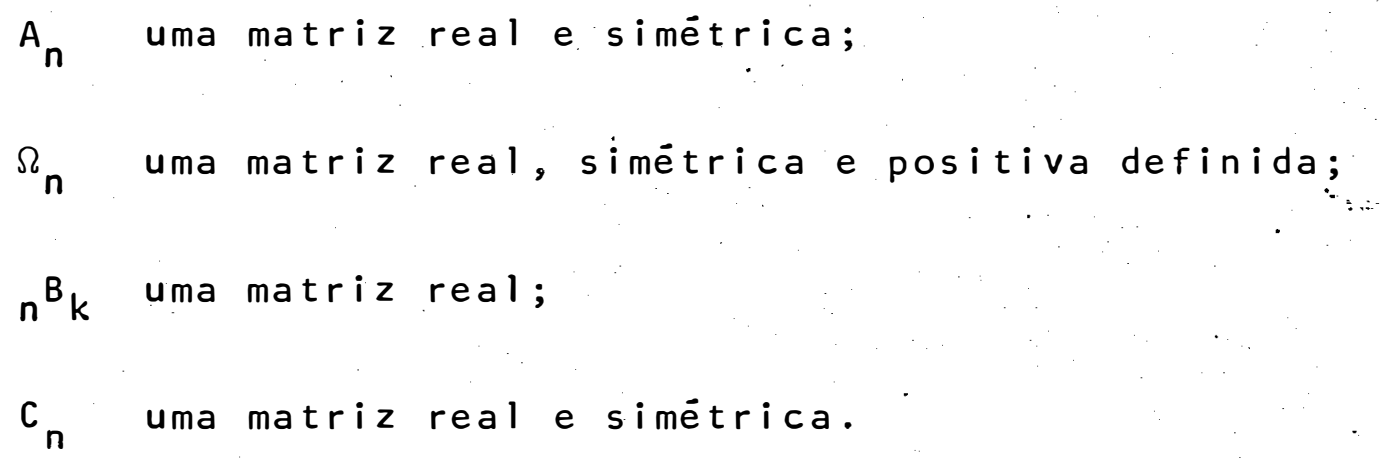

Teorema 5 (Searle, 1971)

i) $E\left[y^{\prime} A y^{\prime}\right]=\operatorname{Tr}[A \Omega] \sigma^{2}+\mu^{\prime} A \mu$.

i i) $\left.\frac{y^{\prime} A y}{\sigma^{2}} \sim x^{2}\left[r(A) ; \frac{1}{2 \sigma^{2}} \mu^{\prime} A \mu\right].\right]$ se e só se $A \Omega$ é idempotente

i i i) y'Ay e y'Cy são independentementè distribuídas se e só $A \Omega C=\emptyset$ ou $C \Omega A=\emptyset$ (s imétricas)

iv) y'Ay e B'y são independentemente distribuídas se e só se $B \Omega A=\emptyset$. 


\section{MÉTODO}

\subsection{INTRODUÇÃO}

Para a compreensão de resultados oriundos do uso da Decomposição por Valores Singulares, é necessário abordar alguns aspectos básicos da teoria da Decomposição pelo Valor Singular de uma matriz X. Nesse contexto, a proprie dade sobre a qual discorre-se a seguir é fundamental para os propósitos do presente estudo, e a sua prova segue as diretrizes apresentadas, com riquezas de detalhes, em CARVALHO $(1982)$

\subsection{A DECOMPOS IÇÃO DA MATRIZ $X$ POR VALORES SINGULARES}

Segundo Eckart e Young (1936), citado por. CARVALHO (1982), dada uma matriz ${ }_{n}{ }_{p}$ de rank $r \leqq m i n\{n, p\}$ com valores singulares $s_{1} \geqq s_{2} \geqq \ldots s_{r}$, existem matrizes orto togonais ${ }_{n} u_{n}$ e ${ }_{p} v_{n}$ tais que

$$
n^{X}=n_{n} n^{S} n n^{V^{\prime}} p
$$

Eckart, C. e Young, G. The aproximation of a matrix by another of lower rank. Psychometrika, (1): 211-218, 1936. 
onde

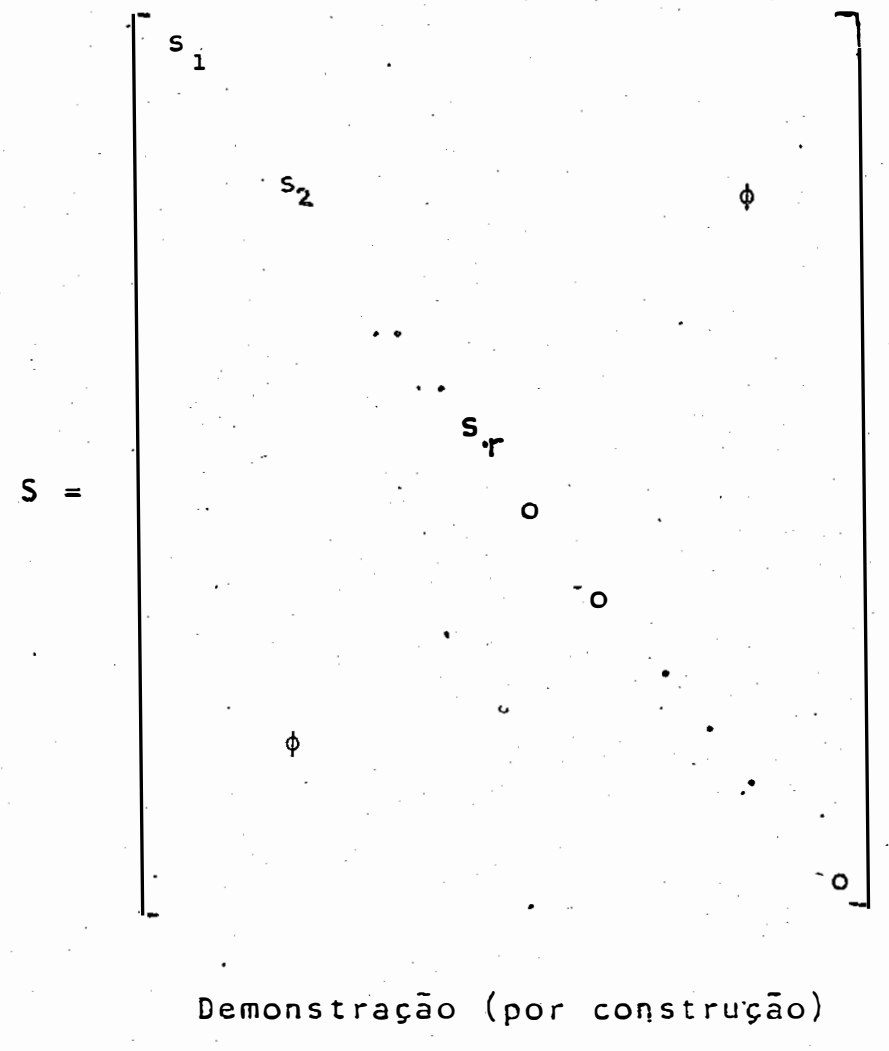

i) $0 \mathrm{~s}$ vetores $u_{1}, \mathrm{u}_{2}, \ldots, \mathrm{u}_{r}, \mathrm{u}_{r+1} \ldots, \mathrm{u}_{n}$, as $n$ colunas de U, são escolhidas como um conjunto de autovetores ortogo nais normalizados de ${ }_{n} X^{\prime}{ }_{n}$, correspondendo aos autovalo $\operatorname{res} s_{1}^{2}, s_{2}^{2}, \ldots, s_{r}^{2}, 0,0, \ldots, 0$ de $x x^{\prime} ;$ toma-se então

$$
U=\left[U_{1}: U_{2}\right] ; \text { onde }
$$

$U_{1}=\left[u_{1}, u_{2}, \ldots, u_{r}\right]$ são os autovetores normalizados correspondentes aos autovalores não nulos de $X X^{\prime} ; s_{1}^{2}, s_{2}^{2}, \ldots s_{r}^{2} ; e$ 
$U_{2}=\left[u_{r+1}, u_{r+2}, \ldots, u_{n}\right]$ são os autovetores corresponden tes aos autovalores nulos de $X x^{\prime} ; s_{r+1}^{2}, s_{r+2}^{2}, \ldots, s_{n}^{2}$.

Seja $U_{I}$ um conjunto ortonormal de autovetores de $X X^{\prime}$, correspondente aos autovalores não nulos.

$$
\begin{gathered}
X X u_{i}=s_{i}^{2} u_{i} ; i=1,2, \ldots, r \\
\text { CARVALHO }(1982) \text { define } v_{i} ; i=1,2, \ldots, r \text { por } \\
v_{i}=\frac{1}{s_{i}} X^{\prime} u_{i}
\end{gathered}
$$

tendo-se então:

$$
\begin{aligned}
v_{i}^{i} v_{j} & =\frac{1}{s_{i} s_{j}}\left(x u_{i}\right){ }^{\prime}\left(x^{\prime} u_{j}\right) \\
& =\frac{1}{s_{i} s_{j}} u_{i}^{1} x x^{\prime} u_{j} ; \\
& =\frac{1}{s_{i} s_{j}} u_{i}^{1} s_{j}^{2} u_{j} \\
v_{i}^{i} v_{j} & =\frac{s_{j}}{s_{i}} u_{i}^{\prime} u_{j} ; \text { mas os } u_{i} \text { são ortogonais, en }
\end{aligned}
$$

tão:

$$
v_{i}^{i} v_{j}=\frac{s_{i}}{s_{j}} \delta_{i j}=\delta_{i j} \text {, onde } \delta_{i j} \text { refere-se ao } \delta \text { de }
$$

Kronecker; portanto $v_{i}, 1 \leqq i \leqq r$ são ortogonais. 


$$
\left\{u_{1}, u_{2}, \ldots, u_{r}\right\} \text { constitue uma base }
$$
ortonormal para $c(x),\left\{v_{1}, v_{2}, \ldots, v_{r}\right\}$ onde $v_{i}=\left(1 / s_{i}\right) x^{\prime} u_{j}$, constitue uma base ortonormal para $c\left(X^{\prime}\right)$, $\left\{u_{r+1}, u_{r+2}, \ldots, u_{n}\right\}$ constitue uma base ortonormal pa $r a c^{1}(x)$ e $\left\{v_{r+1}, v_{r+2}, \ldots, v_{. p}\right\}$ constitue uma base ortonormal para $c^{1}\left(x^{\prime}\right)$.

Tem-se então:

$$
\begin{gathered}
u_{1}=\left(u_{1}, u_{2}, \ldots, u_{r}\right) ; u_{2}=\left(u_{r+1}, u_{r+2}, \ldots, u_{n}\right) \\
u=\left[u_{1} ; u_{2}\right] \\
v_{1}=\left(v_{1}, v_{2}, \ldots, v_{r}\right) ; v_{2}=\left(v_{r+1}, v_{r+2}, \ldots, v_{p}\right) \\
v=\left[v_{1} ; v_{2}\right] .
\end{gathered}
$$

tem-se então, por construção:

a) $U=\left[\begin{array}{l:l}U_{1} & U_{2}\end{array}\right]$. e $V=\left[\begin{array}{l:l}V_{1} & V_{2}\end{array}\right]$ são ortogonais

b) $X V_{2}=\phi \quad$ e $\quad X^{1} U_{2}=\phi$

$$
\begin{aligned}
& \text { Seja } S=U^{\prime} X V=s_{i j}, \text { então }
\end{aligned}
$$

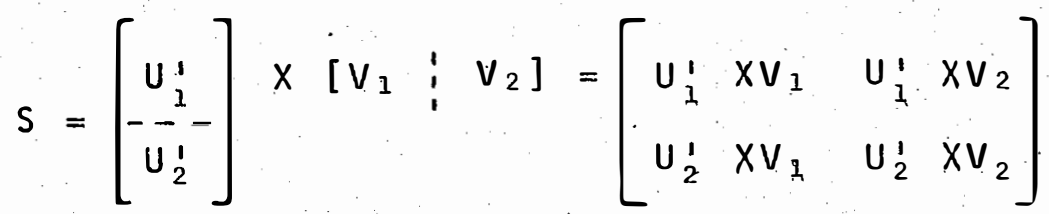

$$
\begin{aligned}
& S=\left[\begin{array}{ccc}
U_{1}^{\prime} & X V_{1} & \phi \\
& \phi & \phi
\end{array}\right]=\left[\begin{array}{ll}
S_{1} & \phi \\
\phi & \phi
\end{array}\right]
\end{aligned}
$$


Dessa forma:

a) se $i<r \quad v j>r \Rightarrow s_{i . j}=\phi$

b) se $i<r \wedge j<r \Rightarrow s_{i, j}=u_{i}^{i} \cdot x_{j}$, mas $v_{j}=\frac{1}{s_{j}} X^{\prime} u_{j}$

portanto,

$$
\begin{aligned}
& s_{i j}=u_{i} x \times \frac{1}{s_{j}} u_{j} ; \text { mas } x \times u_{j}=s_{j}^{2} u_{j} \\
& s_{i j}=u_{i} \frac{s_{j}^{2}}{s_{j}} u_{j}=s_{j} u_{i}^{\prime} u_{j} \\
& s_{i j}=s_{j} \delta_{i j}, \text { iè : } \\
& s_{i j}= \begin{cases}s_{i j} & \text { se } i=j \\
0 & \text { c.c. }\end{cases}
\end{aligned}
$$

$\log 0:$

$$
S=\left[\begin{array}{cccccc}
s_{1} & & & & \phi \\
& s_{2} & & & \\
& & \ddots & & & \\
& & & s_{r} & \\
& & & & \phi & \\
& & & & \ddots & \\
& & & & \ddots & \phi
\end{array}\right]
$$

- que completa a demonstração. 
A D.V.S: fornece imediatamente as matrizes dos projetores ortogonais em $C(X)$ e $C\left(x^{\prime}\right)$, bem como em seus complementos ortogonais

$$
\begin{aligned}
& P_{X}=U_{1} U_{1}^{\prime} \\
& P_{X^{\prime}}=v_{1} v_{1}^{\prime} \\
& P_{X^{\perp}}=1-U_{1} U_{1}^{\prime} \\
& P_{X^{\prime \perp}}=1-v_{1} v_{1}^{\prime}
\end{aligned}
$$




\title{
4. RESULTADOS E DISCUSSÃO
}

\subsection{INTRODUÇÃO}

\author{
Neste capitulo, apresentam-se as propostas o- \\ riginais desta tese para o estudo dos modelos lineares de \\ Gauss-Markov, no tocante às equações normais, estimação e \\ testes estatisticos, e que formam a base para o estudo dos \\ modelos lineares de G.M. sob o prisma dessa técnica.

\subsection{MODELO LINEAR DE GAUSS-MARKOV}

\subsubsection{CARACTER IZAÇÃO} \\ 0 modelo linear

$$
y=x \theta+e
$$

onde

$$
\begin{aligned}
& n^{Y_{1}} \text { é um vetor aleatörio relativo às observações, } \\
& { }_{n}{ }{ }_{p} \text { é a matriz de planejamento, } \\
& { }_{p}^{\theta} \text { é um vetor de parâmetros, }
\end{aligned}
$$


$n^{e}{ }_{1} \vec{e}$ um vetor aleatörio, que tem distribuição normal com média 0 . e variância $1 \sigma^{2}$, é conhecido como modelo linear de Gauss-Markov.

Como $y_{i . j}$ do modelo [4.1.] são funções lineares de $e_{i j}$, das pressuposições sobre os componentes do modelo, tem-se que:

$$
\begin{aligned}
& E(y)=X \theta \\
& V(y)=1 \sigma^{2} \\
& y \sim N\left(x \theta, \quad 1 \sigma^{2}\right)
\end{aligned}
$$

\subsubsection{SOLUÇÃO DE MINIMOS QUADRAD́OS}

Estaremos aqui buscando vetores $\theta^{0}$, tais que a distância euclidiana $d\left(y, X \theta^{\circ}\right)$ seja mínima.

Formando-se a função

$$
z(\theta)=|| y-x \theta \|^{2}
$$

que nos cumpre minimizar, tem-se então que

$$
z(\theta)=y^{\prime} y-2 \theta^{\prime} x^{\prime} y+\theta^{\prime} x^{\prime} x \theta
$$


Derivando-se em relação a $\theta$, resulta

$$
\frac{d z}{d \theta}=-2 x^{\prime} y+2 x^{\prime} x \theta
$$

Igualando-se a zero obtém-se o śistema de equações normais:

$$
x^{+} x \theta^{0}=x^{+} y
$$

Substituindo-se $X$ pela sua D.V.S., o sistema de equações normais [4.5] serä dado por

$$
\begin{aligned}
& P^{V_{1}} T T^{S_{1}^{2}} T T^{V_{1}} P P^{\theta^{0}}=V_{1} S_{1} U_{1}^{1} y \\
& x=U_{1} S_{1} V_{1} \quad e \\
& x^{3} x=v_{1} s_{1}^{2} v_{1}^{1} \text {. }
\end{aligned}
$$

obs.: $T=\operatorname{posto}(x)$

o sistema de equações. [4.6 ] é consistente.

PROVA:

De acordo com GRAYBILL (1969), o sistema $A x=g$ é consistente se e só se $A^{+}{ }^{+} g=g$.

$$
\text { Tomando-se } A=V_{1} s_{1}^{2} V_{1}^{\prime}=x^{\prime} X \text { e } g=V_{1} S_{1} U_{1}^{\prime} y=x^{\prime} \text { y }
$$

$\operatorname{tem}-\mathrm{se}$ que $\operatorname{rank}[A]=\operatorname{rank}\left[V_{2}\right]=\operatorname{rank}\left[S_{1}^{2}\right]=\operatorname{rank}\left[V_{1}^{1}\right]=T$, por tanto, tem-se que: 
a) $A^{+}=\left[V_{1} S_{1}^{2} V_{1}^{1}\right]^{+}=V_{1}^{1}{ }^{+2}{ }_{1}^{2+} V_{1}^{+}=V_{1}^{1} S_{1}^{-2} V_{1}^{+}$

b) $V_{1}^{+}=\left[V_{1}^{1} V_{1}\right]^{-1} V_{1}^{1}$; mas $V_{1}^{1} V_{1}=I_{(T)} \Rightarrow V_{1}^{+}=V_{1}^{1}$

c) $U_{1}^{+}=U_{1}^{1}\left[U_{1}^{1} U_{1}\right]^{-1} ; \operatorname{mas} U_{1}^{\prime} U_{1}=I_{(T)} \Rightarrow U_{1}^{+}=U_{1}^{\prime}$

donde segue-se que

$$
\begin{aligned}
A A^{+} g & =\left[v_{1} s_{1}^{2} v_{1}^{1}\right]\left[v_{1}^{1+} s_{1}^{-2} v_{1}^{+}\right]\left[v_{1} s_{1} u_{1}^{1} y\right] \\
& =v_{1} s_{1}^{2} v_{1}^{1} v_{1}^{+} s_{1}^{-2} v_{1}^{+} v_{1} s_{1} u_{1}^{1} y \\
& =v_{1} v_{1}^{+} v_{1} s_{1} v_{1}^{\prime} y \\
& =v_{1} s_{1} u_{1}^{\prime} y \\
& =g
\end{aligned}
$$

$\therefore$ o sistema $[4.6]$ é consistente.

$$
\begin{aligned}
& \text { Proposição } 1 \\
& \text { O vetor } \theta^{0}=V_{1} S_{1}^{-1} U_{1}^{1} \text { y é solução do sistema }
\end{aligned}
$$
de equações [4.6].

\section{Prova:}

$$
\text { Sejam } p_{P} P_{1}=V_{1} S_{1}^{2} V_{1}^{1} \text { e } A^{+}=V_{1}^{1+} S_{I}^{-2} V_{1}^{+} \cdot \operatorname{Pre}-m u l-
$$

tiplicando [4.6 ] por $A^{+}$tem-se 


$$
\begin{aligned}
& V_{1}^{+} S_{1}^{-2} V_{1}^{+} V_{1} S_{1}^{2} V_{1}^{1} \theta^{0}=V_{1}^{+} S_{1}^{-2} V_{1}^{+} V_{1} S_{1} U_{1}^{1} y \text {; mas } V_{1}^{1+}=V_{1} \text {, então } \\
& v_{1} v_{1} \theta^{0}=v_{1} s_{1}^{-1} U_{1} y \text {, ou seja } \\
& V_{1} v_{1}^{+} \theta^{0}=V_{1} S_{I}^{-1} U_{1}^{1} y \\
& \text { Pré-multiplicando [4.7. ] por } \mathrm{V}_{1}^{+} \\
& v_{1}^{+} v_{1} v_{1}^{+} \theta^{0}=v_{1}^{+} v_{1} s_{1}^{-1} u_{1}^{1} y \\
& v_{1}^{+} \theta^{0}=v_{1}^{+} v_{1} s_{1}^{-1} u_{1}^{1} y \Rightarrow \theta^{0}=v_{1} s_{1}^{-1} U_{1}^{1} y
\end{aligned}
$$

portanto, $\theta^{0}=V_{1} S_{1}^{-1} U_{1}^{1} y \cdot e \bar{e}$ uma solução para o sistema de equações $[4.6$.$] .$

$$
\begin{aligned}
& \text { Proposição } 2 \\
& V_{1} S_{1}^{-1} U_{1}^{-1} \text { é a inversa generalizada de Moore-Pen }
\end{aligned}
$$

rose de ${ }_{n} x_{p}$.

\section{Prova}

A matriz $\mathrm{P}_{n}^{+}=V_{1} S_{1}^{-1} U_{1}^{i}$ satisfaz as quatro con dições da Definição da inversa de Moore-Penrose. De fato:

$\left.a_{1}\right) x x^{+}=U_{1} S_{1} V_{1}^{1} V_{1} S_{2}^{-1} U_{1}^{1}=U_{1} U_{1}^{1} \Rightarrow x x^{+} e^{-}$simetrica

a 2) $X^{+} X=V_{1} S_{1}^{-1} U_{1}^{1} U_{1} S_{1} V_{1}^{1}=V_{1} V_{1}^{1} \Rightarrow X^{+} X^{-}$é simétrica 


$$
\begin{aligned}
& \left.a_{3}\right) x^{+} x x^{+}=\left(x^{+} x\right) x^{+}=v_{1} v_{1}^{1} v_{1} s_{1}^{-1} u_{1}^{\prime}=v_{1} s_{1}^{-1} u_{1}^{\prime}=x^{+} \\
& \left.a_{4}\right) x x^{+} x=\left(x x^{+}\right) x=u_{1} v_{1}^{1} u_{1} s_{1} v_{1}^{\prime}=u_{1} s_{1} v_{1}^{\prime}=x
\end{aligned}
$$

Definição (IEMMA, 1987 a): Se $\theta^{0}$ : é qualquer solução das E.N., $X^{\prime} X \theta=X^{\prime} y, e^{-}$portanto solução de mínimos quadrados para o sistema inconsistente $y=x \theta$, então $o$ vetor $\hat{y}=x \theta^{0}$ é definido como a aproximação de mínimos quadrados para $y$.

De acordo com $[4.6$ ], substituindo-se $x$ por $U_{1} S_{1} V_{1}^{\prime}$ nas $E . N .$, tem-se:

$$
\begin{aligned}
& V_{1} S_{1}^{2} V_{1}^{\prime} \theta^{0}=V_{1} S_{1} U_{1}^{\prime} y \\
& \text { Be acordo com a proposição } 1, \\
& \theta^{0}=V_{1} S_{1}^{-1} U_{1}^{\prime} y
\end{aligned}
$$

é uma solução paria [4.6.] e por conseguinte,

$$
\hat{y}=x \theta^{0}=U_{1} S_{1} V_{1}^{1} V_{1} S_{1}^{-1} U_{1}^{\prime} y=U_{1} U_{1}^{\prime} y=P_{U_{1}} y[4.8]
$$

è uma aproximação de mín.imos quadrados para y.

Fato: A melhor solução aproxịmada de $A x=g$ inconsistente é dada por $x^{*}=A^{+} g$.

De acordo com a proposição $2, V_{1} S_{1}^{-1} U_{1}^{\prime}=x^{+}$, portanto, $\theta^{0}=V_{1} S_{1}^{-1} U_{1}^{1} y=x^{+} y$. Consequentemente, 
$\theta^{0}=V_{1} S_{1}^{-1} U_{1}^{1} y$ é a melhor solução aproximada (B.A.S.) pa ra $y=X \theta$.

\section{Portanto,}

$$
\hat{y}=x \theta^{0}=x x^{+} y=U_{1} S_{1} v_{1}^{1} v_{1} s_{1}^{-1} U_{1}^{1} y=U_{1} U_{1}^{1} y=P_{U_{1}} y
$$

ou seja

$$
\hat{y}=\dot{P}_{u_{1}} y
$$

è a aproximação de mínimos quadrados para o vetor y, das observações.

$$
\begin{aligned}
& \text { 4.2.3. MÉDIA DE } \theta^{\circ} \\
& \text { De } \theta^{0}=V_{1} S_{1}^{-1} U_{1}^{1} y \text {, obtém-se que } \\
& E\left(\theta^{0}\right)=E\left[V_{1} S_{1}^{-1} U_{1}^{1} y\right] \\
& =V_{1} S_{1}^{-1} U_{1}^{1} E(y) ; \operatorname{mas} E(y)=x_{\theta} \\
& \text { Portanto } \\
& =U_{1} S_{1} V_{1}^{\prime} \theta \text {. } \\
& E\left(\theta^{\circ}\right)=V_{1} S_{1}^{-1} U_{1} U_{1} S_{1} V_{1}^{\prime} \theta \\
& =V_{1} \cdot v_{1}^{\prime} \theta=P_{V_{1}} \theta
\end{aligned}
$$


Fato: Nota-se que $\theta^{\circ}$ é viezado para as funções de $\theta$ que es tão em $P_{V_{1}} \theta$.

\subsubsection{UM ESTIMADOR NÃO TENDENCIOSO PARA $\sigma^{2}$}

Proposição 3 :

No modelo linear de Gauss-Markov,

$$
\bar{\sigma}^{2}=\frac{1}{n-T} y^{\prime} P_{U_{2}} y
$$

è um estịmador não tendencioso para $\sigma^{2}$.
Prova: $\mathrm{PU}_{2}=\left(1-\mathrm{P}_{U_{1}}\right)$ é uma matriz real, simétrica e idem potente., e y $N\left(X \theta, I \sigma^{2}\right)$. Logo,

$$
\begin{aligned}
& \therefore E\left(y^{\prime} P_{U_{2}} y\right)=\operatorname{Tr}\left(P_{U_{2}}\right) I \sigma^{2}+\theta^{\prime} X^{\prime} P_{U_{2}} X \theta \\
& =\operatorname{Tr}\left(P_{U_{2}}\right) 1 \sigma^{2}+\theta^{\prime} V_{1} S_{1} U_{1}^{3} P_{U_{2}} U_{1} S_{1} Y_{1}^{\prime} \theta \\
& =\operatorname{Tr}\left(P_{U_{2}}\right) I \sigma^{2}+\theta^{\prime} V_{1} S_{1} U_{1}^{\prime}\left(1-P_{U_{1}}\right) U_{1} S_{1} V_{1}^{\prime} \theta \\
& =(n-T) \sigma^{2}+\theta^{\prime} V_{1} S_{I}^{2} V_{I}^{\prime} \theta-\theta^{\prime} V_{I} S_{1} U_{I}^{1} P_{U_{1}} U_{1} S_{1} V_{1}^{\prime} \theta \\
& =(n-T) \sigma^{2}+\theta^{\prime} V_{1} S_{1}^{2} V_{1}^{1} \theta-\theta^{\prime} V_{1} S_{1}^{2} V_{1}^{1} \theta \\
& =(n-T) \sigma^{2}
\end{aligned}
$$




$$
\begin{aligned}
E\left[\tilde{\sigma}^{2}\right] & =E\left[\frac{1}{n-T} y^{\prime} P_{U_{2}} y\right] \\
& =\frac{1}{n-T} E\left(y^{\prime} P_{U_{2}} y\right) \\
& =\frac{1}{n-T}(n-T) \sigma^{2} \\
& =\sigma^{2}
\end{aligned}
$$

concluindo a demonstração.

\subsection{5, FUNÇÕES EST IMÁVE IS}

Proposição 4. A função $\lambda^{\prime} \cdot \theta$ è estimāvel no modelo linear de Gauss-Markov se e somente se $\lambda^{\prime} V_{1} V_{1}^{\prime}=\lambda^{\prime}$.

Prova: De acordo com Rao (1945) a função para métrica $\lambda^{\prime} \theta$ é dita estimável no modelo de Gauss-Markov se e só se existe uma combinação linear das observações, a'y, tal que

$$
E\left(a^{\prime} y\right)=\lambda^{\prime} \theta
$$

$\Leftrightarrow) \lambda^{\prime} \theta$ é. estimāvel

então existe uma função linear das observações, a'y, tal que 
$E\left(a^{\prime} y\right)=\lambda \cdot \theta \quad($ Rao, 1945)

ou seja

$$
E\left(a^{\prime} y\right)=a^{\prime} E(y)=a^{\prime} x \theta=\lambda^{\prime} \theta \text {. }
$$

Substituindo $X$ por sua D.l.S. $U_{1} S_{1} V_{1}^{1}$, tem-se para a expressão anterior:

$$
a^{\prime} x \theta=a^{\prime} U_{1} S_{1} V_{1}^{\prime} \theta=\lambda^{\prime} \theta \text {, donde, por serem escala }
$$

res, conclui-se

$$
a^{\prime} U_{1} S_{1} V_{1}^{\prime}=\lambda^{\prime}, \forall \theta .(1 . a)
$$

Pós-multiplicando a expressão obtida por $V_{1} V^{\prime}$,

tem-se: $\quad a^{\prime} U_{1} S_{1} V_{1}^{\prime} V_{1} V_{1}^{\prime}=\lambda V_{1} V_{1}^{\prime}$; simplificando o primeiro membro,

$$
\begin{aligned}
& a^{\prime} U_{1} S_{1} V_{1}^{\prime}=\lambda V_{1} V_{1}^{\prime} \cdot(1 . b) \\
& \text { De (1.a) e (1.b) tem-se então que } \\
& \lambda^{\prime}=\lambda^{\prime} v_{1} v_{1}^{\prime} \\
& \text { (L) } \quad \lambda_{1}=\lambda_{1} v_{1} v_{1}^{\prime} \text {. } \\
& \text { Pós-multiplicando por } \theta \text {, } \\
& \lambda^{\prime} \theta=\lambda^{\prime} V_{1} V_{1}^{\prime} \theta ; \text { De } 4.2 .3 \text {. segue-se: }
\end{aligned}
$$




$$
\lambda^{\prime} \theta=\lambda^{\prime} E\left(\theta^{0}\right)=\lambda^{\prime} E\left(V_{1} S_{1}^{-1} U_{1}^{1} y\right)=\lambda^{\prime} V_{1} S_{1}^{-1} U_{1}^{1} E(y) \text {. Toman- }
$$

do-se

$$
\begin{aligned}
a^{\prime} & =\lambda^{\prime} V_{1} s_{1}^{-1} U_{1}^{\prime}, \text { tem-se } \\
\lambda\lrcorner \theta & =\lambda^{\prime} V_{1} s_{1}^{-1} U_{1}^{\prime} E(y)=a^{\prime} E(y)=E\left(a^{\prime} y\right) \text {. Portanto, exis }
\end{aligned}
$$

te uma função linear das observações, a'y, tal que

$$
E\left(a^{\prime} y\right)=\lambda^{\prime} \theta
$$

Então, de acordo com Rao (1945) $\lambda^{\prime} \theta$ é estimä-

vel.

4.2.6. OBTENCÃO DO B,L,U,E.

De acordo com o teorema de Gauss-Markov, ' o

B.L.U.E. (Best Linear Unbiesed Estimator) de $\lambda^{\prime} \theta$ estimável é dado por

$$
\widehat{\lambda^{\prime} \theta}=\lambda^{1} \theta^{0}
$$

onde $\theta^{0}$ é qualquer solução do sistema de equações normaís do modelo linear de Gauss-Markov

$$
\text { para } \theta^{0}=v_{2} s_{1}^{-1} u_{1}^{1} y \text {, }
$$

verifica-se então que: 


$$
\text { i) } \begin{aligned}
E\left[\lambda^{\prime} \theta^{0}\right]= & E\left[\lambda^{\prime} V_{1} S_{1}^{-1} V_{1}^{\prime} y\right] \\
= & \lambda^{\prime} v_{1} S_{1}^{-1} U_{1}^{\prime} E(y) \\
= & \lambda^{\prime} v_{1} s_{1}^{-1} U_{-1}^{\prime} U_{1} S_{1} v_{1}^{\prime} \theta \\
= & \lambda^{\prime} v_{1} v_{1}^{\prime} \theta ; \text { de acordo com a proposição } 4, \\
& \quad \lambda^{\prime} v_{1} v_{1}^{\prime}=\lambda^{\prime}
\end{aligned}
$$

$\therefore E\left[\lambda \cdot \theta^{\circ}\right]=\lambda^{\prime} \theta$

i i) $V\left[\lambda \cdot \theta^{0}\right]=\lambda \cdot v\left[\theta^{0}\right] \lambda$

$$
\begin{aligned}
& =\lambda^{\prime} V\left[V_{1} S_{1}^{-1} U_{1}^{\prime} y\right] \lambda \\
& =\lambda^{\prime} V_{1} S_{1}^{-1} U_{1}^{\prime} V(y) U_{1} \dot{S}_{1} V_{1}^{\prime} \lambda ; \text { mas } V(y)^{\cdot}=1 \sigma^{2}
\end{aligned}
$$$$
\therefore U\left[\lambda^{\prime} \theta^{\circ}\right]=\lambda^{\prime} V_{1} s_{1}^{-1} U_{1}^{1} l \sigma^{2} U_{1} s_{1} v_{1}^{\prime} \lambda
$$$$
=\lambda \cdot V_{1} S_{1}^{-2} V_{1}^{\prime} \lambda \sigma^{2}
$$

\subsection{Sistema DE Equações NORNiA IS REDUZidas}

\subsubsection{O MODELO LINEAR}

Tomando-se a partição

$$
x=\left[\begin{array}{l:ll}
x_{1} & x_{2}
\end{array}\right]
$$


tem-se o modelo linear

$$
y=\left[\begin{array}{l:l}
x_{1} & x_{2}
\end{array}\right]\left[\begin{array}{c}
\theta_{1} \\
\hdashline \\
\theta_{2}
\end{array}\right]+e
$$

ou. seja

$$
y=x_{1} \theta_{1}+x_{2} \theta_{2}+e .
$$

As equações normais, convenientemente parti

das, resultam

$$
\begin{aligned}
& x_{1}^{1} x_{1} \theta_{1}+x_{1}^{1} x_{2} \theta_{2}=x_{1}^{1} y \\
& x_{2}^{1} x_{1} \theta_{1}+x_{2}^{1} x_{2} \theta_{2}=x_{2}^{1} y
\end{aligned}
$$

prë-multiplicando $[4.11]$ por $x_{2}^{\prime} x_{1}^{1+}$, vem:

$$
x_{2}^{1} x_{1}^{1+} x_{1}^{\prime} x_{1} \theta_{1}+x_{2}^{1} x_{1}^{1+} x_{1}^{1} x_{2} \theta_{2}=x_{2}^{1} x_{1}^{1+} x_{1}^{1} y
$$

de acordo com as propriedades da inversa generalizada de Moo re-Penrose, tem-se que;

$$
\begin{aligned}
& x_{2}^{\prime} x_{1} x_{1}^{+} x_{1} \theta_{1}+x_{2}^{\prime} x_{1} x_{1}^{+} x_{2} \theta_{2}=x_{2}^{1} x_{1} x_{1}^{+} y, \\
& x_{2}^{\prime} x_{1} \theta_{2}+x_{2}^{1} x_{1} x_{1}^{+} x_{2} \theta_{2}=x_{2}^{\prime} x_{1} x_{1}^{+} y,
\end{aligned}
$$

de acordo com o teorema $3, X_{1} X_{1}^{+}=P_{X_{1}}$; portanto

$$
x_{2}^{\prime} x_{1} \theta_{1}+x_{2}^{\prime} P_{x_{1}} x_{2} \theta_{2}=x_{2}^{1} \cdot P_{x_{1}}^{y}
$$




$$
\begin{gathered}
\text { Subtraindo [4.13.] de [4.12;], } \\
X_{2}^{\prime} X_{1} \theta_{1}-X_{2}^{\prime} X_{1} \theta_{1}+X_{2}^{\prime} X_{2} \theta_{2}=x_{2}^{\prime} P_{X_{1}} x_{2} \theta_{2}=X_{2}^{\prime} y-X_{2}^{\prime} P_{X_{1}} y
\end{gathered}
$$

que resultarā:

$$
x_{2}^{\prime}\left(1-P_{x_{1}}\right) x_{2} \theta_{2}=x_{2}^{\prime}\left(1-P_{x_{1}}\right) y_{1}[4.14]
$$

que é o sistema de equações normais reduzidas, eliminando o efeito do parâmetro $\theta_{1}$.

De acordo com IEMMA (1985), o sistema [4.14.] é consistente, visto que tem a mesma estrutura de [4.2]. Definindo-se:

$$
\begin{aligned}
u_{1 i}(i=1,2) \quad\left[v_{1 i}(i=1,2)\right] & \text { a matriz formada pelos } \\
& \text { autovetores ortonormais } \\
& \text { de } x_{i} x_{i}(i=1,2)\left[x_{i} x_{i}\right. \\
& (i=1,2)] \text { corresponden } \\
& \text { tes aos autovalores não } \\
& \text { nulos de } x_{i} x_{i}(i=1,2) \\
& {\left[x_{i} x_{i}(i=1,2)\right] }
\end{aligned}
$$

$s_{1 i}(i=1,2)$ a matriz formada pelos autovalores não nu los da matriz $x_{i} x_{i}^{\prime}(i=1,2)$ e $\quad x_{i} x_{i}$ $(i=1,2)$, respectivamente. 


$$
\text { da matriz } x_{2} x_{2}^{\prime}\left(X_{2}^{\prime} x_{2}\right. \text {, respectivamente); }
$$

tem-se então, sem perda de generalidade, que:
a) $x_{1}=U_{12} S_{12} v_{11}^{\prime}$
b) $x_{1}^{+}=v_{11} s_{11}^{-1} \quad u_{11}^{1}$
c) $P_{X_{1}}=x_{1} x_{1}^{+}=U_{12} U_{12}^{1}=P_{U_{11}}$
d) $x_{2}=U_{12} S_{12} V_{12}^{\prime}$
e) $x_{2}^{+}=v_{12} s_{12}^{-1} \quad u_{12}^{1}$
f) $P_{X_{2}}=X_{2}^{+} X_{2}=U_{12} \quad U_{12}^{\prime}=P_{U_{12}}$

pelo enfoque D.V.S., tem-se por [4.14]

$$
v_{12} s_{12} U_{12}^{\prime}\left(1-P_{U_{11}}\right) u_{12} s_{12} v_{12}^{\prime} \theta_{2}=v_{12} s_{12} u_{12}^{\prime}\left(1-P_{U_{11}}\right) y
$$

tomando-se $A=V_{12} S_{12} U_{12}^{1}$, teremos $A^{+}=U_{12}^{1+} S_{12}^{-1} V_{12}^{+}$

$$
\text { De acordo com a teoria das inversas generali- }
$$

zadas de Moore-Penrose, obtém-se que:

$$
\begin{aligned}
& v_{12}^{+}=v_{12}^{1} \quad\left(v_{12}^{+}=v_{12}\right) \\
& u_{12}^{+}=u_{12}^{1} \quad\left(u_{12}^{+}=u_{12}\right)
\end{aligned}
$$

pré-multiplicando ambos os lados de [4.15] por $A^{+}$tem-se 
.40 .

$$
\begin{gathered}
U_{12}^{+} s_{12}^{-1} v_{12}^{+} v_{12} s_{12} U_{12}^{\prime}\left(1-P_{U_{11}}\right) x_{2} \theta_{2}=U_{12}^{+} s_{12}^{-1} v_{12}^{+} v_{12} s_{12} U_{12}^{\prime}\left(1-P_{U_{11}}\right) y \\
U_{12}^{+} U_{12}^{\prime}\left(1-P_{U_{11}}\right) x_{2} \theta_{2}=U_{12}^{+} U_{12}^{\prime}\left(1-P_{U_{11}}\right) y
\end{gathered}
$$

$$
\text { por }[4.17]
$$

$$
U_{12} U_{12}^{\prime}\left(1-P_{U_{11}}\right) x_{2} \theta_{2}=U_{12} U_{12}^{1}\left(1-P_{U_{11}}\right) y^{\prime}
$$

$\operatorname{mas}\left(1-P_{U_{11}}\right)=P_{X} \frac{1}{I}=U_{21} U_{21}^{1}=P_{U_{21}}$,

onde $U_{21}$ é a matriz formáda pelos autovetores ortonormais cor respondentes aos autovalores nulos de $X_{1} X_{1}$; tem-se então pa ra $[4.18]$

$$
P_{U_{I 2}} P_{U_{21}} X_{2} \theta_{2}=P_{U_{12}} P_{U_{21}} Y
$$

é o sistema de equações normais reduzidas, eliminando o efe to do parâmetro $\theta_{1}$, substituindo-se $X_{1}$ pela sua D.V.S.

Nas expressões [4.18] e [4.19] optou-se em não substituir $X_{2}$ pela sua D.V.S. para que as expressões ficassem reduzidas às formas mais simples.

$$
\begin{aligned}
& \text { De maneira anāloga, } \\
& P_{U_{21}} P_{U_{12}} X_{1} \theta_{1}=P_{U_{21}} P_{U_{12}} Y
\end{aligned}
$$

è o sistema de equações normais reduzidas eliminando o efeito do parâmetro $\theta_{2}$ e substituindo $X_{2}$ e $X_{2}$ pelas respectivas D.V.S. 


\subsubsection{ESTIMABILIDADE DE UM SUBCONJUNTO DE PARÂME- TROS}

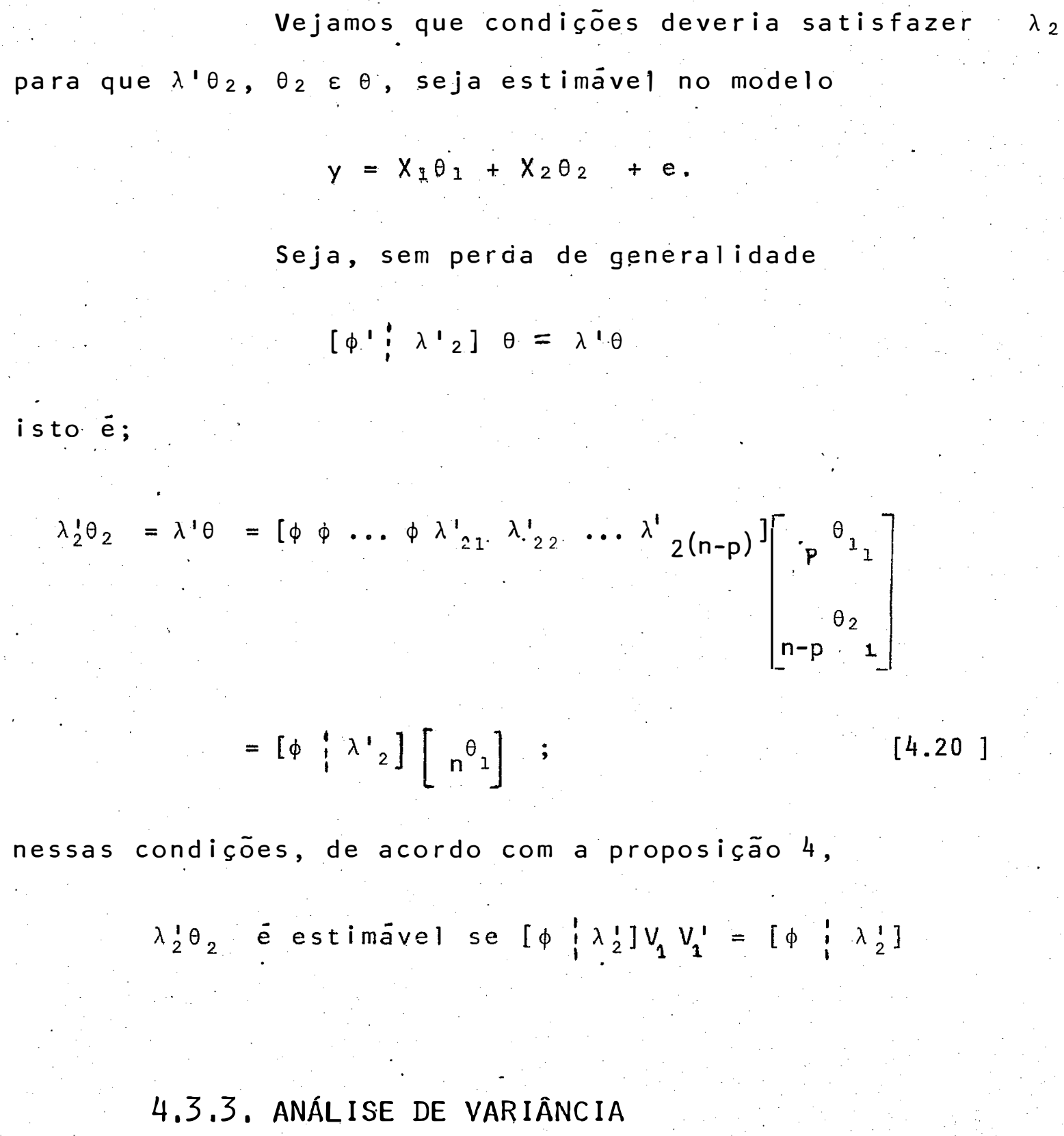

nessas condições, de acordo com a proposição 4 ,

$\lambda_{2}^{\prime} \theta_{2}$ é estimävel se $\left[\phi \lambda_{2}^{\prime}\right] V_{1} V_{1}^{\prime}=\left[\begin{array}{lll}\phi & \lambda & 1\end{array}\right]$

\subsubsection{ANÁL ISE DE VARIÂNC IA}

Suponha-se, de inicio, que o modelo esteja par- 
ticionado.

$$
\begin{aligned}
& y=x_{1} \theta_{1}+x_{2} \theta_{2}+e \\
& \text { Seja } x=\left(x_{1} ; x_{2}\right) ; \quad \theta=\left[\begin{array}{c}
\theta_{1} \\
\theta \\
2
\end{array}\right]
\end{aligned}
$$

tomando-se como base o submodelo

$$
y=x_{1} \theta_{1}+e
$$

tem-se que

i) Existe $P_{U_{11}}=U_{11} U_{11}^{1}$ tal que $x_{1} \theta_{1}^{0}=P_{U_{1 I}} Y$

i i). $\left\|y-x_{1} \theta_{1}^{0}\right\|^{2}=y^{\prime} y-y^{\prime} x_{1} \theta_{1}^{0}$

$$
\begin{aligned}
=y^{\prime} y-y^{\prime} P_{U_{11}} y \\
\therefore \quad y^{\prime} y=y^{\prime} P_{U_{11}} y+\left\|y-x_{1} \theta_{1}^{0}\right\|^{2} \\
=y^{\prime} P_{U_{11}} y+y^{\prime} y-y^{\prime} P_{U_{11}} y \\
=y^{\prime} P_{U_{11}} y+y^{\prime}\left(1-P_{U_{11}}\right) y
\end{aligned}
$$

onde $y^{\prime} P_{U_{1} I} y$ é a soma de quadrados devido a $\theta_{1}$

$$
\begin{array}{r}
y^{\prime}\left(1-P_{U_{1}}\right) y \text { é a soma de quadrados devido ao erro de a } \\
\text { justamento a través do submodelo adotado. }
\end{array}
$$

Tomando-se o modelo completo, tem-se agora que:

i i i) $P_{U_{1}}=U_{1} U_{1}^{\prime}$ 
iv) $\left\|y-x \theta^{0} \quad\right\|^{2}=y^{1} y-y^{3} x \theta^{0}$

$$
\begin{gathered}
=y^{\prime} y-y^{\prime} P_{U_{7}} y^{\prime} \\
\therefore y^{\prime} y=y^{\prime} P_{U_{I}} y+y^{\prime}\left(1-P_{U_{I}}\right) y ;
\end{gathered}
$$

da invariancia de $y^{\prime} y$ tem-se:

$$
\begin{aligned}
& y^{\prime} P_{U_{1}} y+y^{\prime}\left(1-P_{U_{1}}\right) y=y^{\prime} P_{U_{11}} y+y^{\prime}\left(1-P_{U_{11}}\right) y \\
& y^{\prime} P_{U_{1}} y+y^{\prime} y-y^{\prime} P_{U_{1}} y=y^{\prime} P_{U_{11}} y+y^{\prime} y-y^{\prime} P_{U_{11}} y
\end{aligned}
$$

Reagrupando tem-se

$$
\begin{aligned}
y^{\prime} y & =y^{\prime} P_{U_{11}} y+y^{\prime} P_{U_{1}} y-y^{\prime} P_{U_{11}} y-y^{\prime} P_{U_{1}} y+y^{\prime} y \\
\therefore y^{\prime} y & =y^{\prime} P_{U_{11}} y+y^{\prime}\left(P_{U_{1}}-P_{U_{11}}\right) y+y^{\prime}\left(1-P_{U_{1}}\right) y
\end{aligned}
$$

è comum tambēm a forma

$$
y^{\prime}\left(1-P_{U_{1}}\right) y=y^{\prime}\left(P_{U_{1}}-P_{U_{1 I}}\right) y+y^{\prime}\left(1-P_{U_{1}}\right) y .
$$

De-acordo com GRAYBILL (1969), se $n^{A}{ }_{n}$ e ${ }_{n}{ }^{B}$ são matrizes simétricas e idempotentes, então $A$ - B é uma matriz simétrica e idempotente se e somente se

$$
B(A-B)=\phi
$$

Fato: $P_{U_{2}}$ e $P_{U_{1} 1}$ são matrizes simétricas e idempotentes.

De fato:

$$
P_{U_{1}} P_{U_{1}}=U_{1} U_{1}^{1} U_{1} U_{1}^{1}=U_{1} U_{1}^{1}=P_{U_{1}}
$$




$$
\begin{aligned}
& P_{U_{11}} P_{U_{11}}=U_{11}^{U_{11}^{\prime}} U_{11} U_{11}^{\prime}=U_{11} U_{11}^{\prime}=P_{U_{11}} \\
& P_{U_{1}}=U_{1} U_{1}^{\prime}=\left(U_{1}^{U_{1}^{\prime}}\right)^{\prime}=P_{U_{1}}^{\prime} \\
& P_{U_{11}}=U_{11}^{U_{11}^{\prime}}=\left(U_{11} U_{11}^{\prime}\right)^{\prime}=P_{U_{11}^{\prime}}^{\prime}
\end{aligned}
$$

Obs.:

$$
\begin{aligned}
& P_{U_{11}}\left(P_{U_{I}}-P_{U_{11}}\right)=P_{U_{1 I}} P_{U_{1}}-P_{U_{1 I}} P_{U_{11}} \\
& =P_{U_{I}{ }_{I}} P_{U_{I}}-P_{U_{I I}} ; \text { visto que } \\
& P_{U_{1}} \bar{e} \text { o projetor ortogonal } \\
& \text { em } C(X) \text { e } P_{U_{I I}} \text { é o proje- } \\
& \text { tor ortogonal em } C\left(X_{1}\right) \text { e } \\
& x_{1}<x, \text { tem-se que: } \\
& P_{U_{11}}\left(P_{U_{1}}-P_{U_{11}}\right)=P_{U_{11}}-P_{U_{11}} \\
& =\phi \\
& \therefore \quad\left(P_{U_{1}}-P_{U_{1}}\right)^{\prime} e^{-} \text {simētrica e idempotente. } \\
& \text { Pode-se então construir o quadro da Anālise }
\end{aligned}
$$

de Variància. 
Quadro 1 - Anāli se de Variância

\begin{tabular}{lcc}
\hline CV & G.L. & SQ \\
\hline Devido a $\theta_{1}$ & $r\left(U_{11}\right)$ & $y^{\prime} P_{U_{11}} y$ \\
Devido a $\theta_{2} / \theta_{1}$ & $r\left(U_{1}\right)-r\left(U_{11}\right)$ & $y^{\prime}\left(P_{U_{1}}-P_{U_{11}}\right) y$ \\
Residuo & $n-r\left(U_{1}\right)$ & $y^{\prime}\left(1-P_{U_{1}}\right) y$ \\
\hline Total & $n$ & $y^{\prime} y$ \\
\hline
\end{tabular}

4.4. DISTRIBÚIÇÃO DAS FORMAS QUADRÁTICAS

4.4.1. INTRODUÇÃO

$$
\begin{aligned}
& \text { Uma função do tipo } \\
& Q=-\sum_{i} \sum_{j} a_{i j} x_{i} x_{j} \text {, onde }
\end{aligned}
$$

$a_{i j}$ são constantes reais, é definida como uma forma quadräti ca de n variāveis.

Sob a forma matricial, uma forma quadrática é escrita por 


$$
Q=x^{\prime} A x^{\prime} \text {, onde }
$$

$n^{x_{i}}$ è um vetor de variäveis

$n^{A}{ }_{n}$ é a matriz ou nūcleo da forma quadrätica.

Estudam-se a seguir as distribuições das formas quadräticas de interesse, sob as condições de Gauss-Markov.

\subsubsection{A DISTRIBUICÃO DE Y'P $\mathrm{P}_{I} Y$}

Sendo a matriz $P_{U_{1}}$ real, simétrica e idempo- tente então, de acordo com o Teorema 5 , item (i) e (ii)-ca pítulo 2, tem-se que:

$$
E\left(y^{\prime} P_{U_{1}} y\right)=\operatorname{Tr}\left(P_{U_{1}}\right) \sigma^{2}+\theta^{\prime} V_{1} S_{1}^{2} V_{1}^{\prime} \theta \quad e
$$

$$
\frac{y^{\prime} P_{U_{1}} y}{\sigma^{2}} \sim x^{2}\left[r\left(P_{U_{1}}\right) ; \frac{1}{2 \sigma^{2}} \theta^{\prime} V_{1} s_{1}^{2} V_{1} \theta\right] \text {, respectivamente. }
$$




$$
\begin{aligned}
& \text { 4.4.3. A DISTRIBUICÃ̃O DE } y^{\prime}\left(1-P_{U_{1}}\right) y \\
& E\left(y^{\prime}\left(1-P_{U_{1}}\right) y\right)=\operatorname{tr}\left(1-P_{U_{1}}\right) \sigma^{2}+\theta^{\prime} V_{1} S_{1} U_{1}^{\prime}\left(1-P_{U_{1}}\right) U_{1} S_{1} V_{1}^{\prime} \theta \\
& =(n-T) \sigma^{2}+\theta^{\prime} V_{1} S_{1}^{2} V_{1}^{\prime} \theta-\theta^{\prime} V_{1} S_{1} U_{1}^{\prime} P_{U_{1}} U_{1} S_{1} V_{1}^{\prime} \theta \\
& =(n-T) \sigma^{2}+\theta^{\prime} V_{1} S_{1} V_{1}^{\prime} \theta-\theta^{\prime} V_{1} S_{1} V_{1}^{\prime} \theta \\
& =(n-T) \sigma^{2} ;
\end{aligned}
$$

então:

$$
\frac{y^{\prime}\left(1-P_{U_{1}}\right) y}{\sigma^{2}} \approx x^{2}{ }_{[n-T]}
$$

$$
\text { 4.4.4. A DISTRIBUICÃO DE y! }\left(P_{U_{1}}-P_{U_{11}}\right) y
$$

Segundo GRAYBILL (1969), se a matriz (A-B) è simétrica e idempotente, então $\operatorname{tr}(A-B)=\operatorname{tr}(A)-\operatorname{tr}(B)$.

Tem-se então que :

$$
\begin{aligned}
E\left(y^{\prime}\left(P_{U_{1}}-P_{U_{11}}\right) y\right) & =\operatorname{tr}\left(P_{U_{1}}-P_{U_{11}}\right) \sigma^{2}+\theta^{\prime} V_{1} S_{1} U_{1}^{\prime}\left(P_{U_{1}}-P_{U_{11}}\right) U_{1} S_{1} V_{1}^{\prime} \theta \\
= & {\left[\operatorname{tr}\left(P_{U_{1}}\right)-\operatorname{tr}\left(P_{U_{11}}\right)\right] \sigma^{2}+\theta^{\prime} V_{1} S_{1}^{2} V_{1}^{\prime} \theta^{\prime}-\theta^{\prime} V_{1} S_{1} U_{1}^{\prime} P_{U_{11}} U_{1} S_{1} V_{1}^{\prime} \theta } \\
= & {\left[T-\operatorname{tr}\left(P_{U_{11}}\right)\right] \sigma^{2}+\theta^{\prime} V_{1} S_{1}^{2} V_{1}^{\prime} \theta-\theta^{\prime} V_{1} S_{1} U_{1}^{\prime} P_{U_{11}} U_{1} S_{1} V_{1}^{\prime} \theta }
\end{aligned}
$$


então

$$
\begin{aligned}
& y^{\prime}\left(P_{U_{1}}-P_{U_{21}}\right) y \\
& \sigma^{2} \quad\left[T-\operatorname{tr}\left(P_{U_{11}}\right) ; \frac{1}{2 \sigma^{2}}\left[\theta^{\prime} V_{1} S_{1}^{2} V_{1}^{\prime} \theta-\theta^{\prime} V_{1} S_{1} U_{1}^{\prime} P_{U_{11}} U_{1} S_{1} V_{1}^{\prime} \theta\right]\right] \\
& \text { 4.4.5. A DISTRIBUICÃO DE } y^{\cdot 1}\left(1-P_{U_{11}}\right) y \\
& E\left[y^{\prime}\left(1-P_{U_{1 I}}\right) y\right]=\operatorname{tr}\left(1-P_{U_{1 I}}\right) \sigma^{2}+\theta^{\prime} V_{I} S_{1} U_{1}^{\prime}\left(1-P_{U_{1 I}}\right) \dot{U}_{1} S_{1} V_{1}^{\prime} \theta \\
& \left.=\left[n-\operatorname{tr}\left(P_{U_{1} I}\right)\right] \sigma^{2}+\theta^{\prime} V_{1} S_{1}^{2} V_{1}^{\prime} \theta-\theta^{\prime} V_{I} S_{1} U_{1}^{\prime} P_{U_{1}} U_{I} S_{1} V_{1}^{\prime} \theta\right] \\
& \text { e } \\
& \frac{y^{\prime}\left(1-P_{U_{11}}\right) y}{\sigma^{2}} \sim x^{2} \\
& {\left[n-\operatorname{tr}\left(P_{U_{11}}\right) ; \frac{1}{2 \sigma^{2}}\left(\theta^{\prime} V_{1} S_{1}^{2} V_{1}^{\prime} \theta-\theta^{\prime} V_{1} S_{1} U_{1}^{\prime} P_{U_{I I}} U_{1} S_{I} V_{1}^{\prime} \theta\right)\right] \quad[4.23]}
\end{aligned}
$$

\subsection{INTERVALOS E REgIỘES DE CONFIANCA}

No modelo de Gauss-Markov

$$
y=x \theta+e \text {, }
$$

$$
\begin{aligned}
\text { onde } E(e)=\phi e \\
\therefore \quad E\left(e^{\prime} e\right)=1 \sigma^{2},
\end{aligned}
$$


$\widehat{\lambda^{\prime} \theta}=\lambda^{\prime} \theta^{0}=\lambda^{\prime} V_{I} S_{I}^{-1} U_{i}^{\prime} y$ è uma combinação linear de variàveis normais, sendo portanto normal.

\subsubsection{INTERVALOS DE CONFIANCA}

$$
\begin{aligned}
& \text { De acordo com }[4.9] \text { e }[4.10], \text { tem-se que } \\
& \widehat{\lambda^{\top} \theta} \sim N\left(\lambda^{+} \theta ; \lambda^{1} V_{1} S_{1}^{-2} V_{1}^{1} \lambda \sigma^{2}\right) .
\end{aligned}
$$

Definindo-se:

$$
\begin{aligned}
& =\frac{\widehat{\lambda^{\prime} \theta}-\lambda^{\prime} \theta}{\sigma\left[\lambda^{\prime} v_{1} s_{1}^{-2} V_{1}^{\prime} \lambda\right]^{1 / 2}} \text {; tem -se que } \\
& E(Z)=\frac{1}{\sigma\left[\lambda^{\prime} V_{1} s_{1}^{2} V_{1}^{\prime} \lambda\right]^{1 / 2}} E\left[\widehat{\lambda^{\prime} \theta}-\lambda^{\prime} \theta\right]=\phi \\
& V[Z]=\frac{1}{\sigma^{2} \lambda^{\prime} V_{1} S_{1}^{-2} V_{1}^{\prime} \lambda} v\left[\widehat{\lambda^{\prime} \theta}-\lambda{ }^{\prime} \theta\right] \\
& =\frac{\sigma^{2} \lambda^{1} V_{1} S_{1}^{-2} V_{1}^{\prime} \lambda}{\sigma^{2} \lambda^{\prime} V_{1} s_{1}^{-2} V_{1}^{\prime} \lambda}=1
\end{aligned}
$$

Desse modo, $Z \sim N(0,1)$;

$$
\text { De acordo com IEMMA (1982), } \lambda^{\prime} \theta \text { e } y^{\prime}(1-P) y, \underline{\underline{n}}
$$

de P. é o projetor ortogonal de $y \mathrm{em}^{-} \mathrm{C}(\mathrm{X})$, são independentes. 
Definição (SEARLE, 1982): Seja o modelo GaussMarkov Normal, com ${ }_{n} y_{z}$ e $r(x)=T$, então

$$
\frac{(n-T) \hat{\sigma}^{2}}{\sigma^{2}}=\frac{y^{\prime}(l-P) y}{\sigma^{2}} \sim x^{2}(n-T)
$$

Definição (SEARLE, 1982): Dadas as variāveis aleatörias $Z \sim N(0,1)$ e $W \sim x^{2}(n)$, independentes, então a va riāvel aleatöria $M$, onde.

$$
\begin{aligned}
& M=\frac{Z}{\sqrt{\frac{W}{n}}}, \text { tem distribuição } t \text { de Student com } n \text { graus } \\
& \text { Então, para o caso em questão: } \\
& M=\frac{\frac{\widehat{\lambda^{\prime} \theta}-\lambda^{\prime} \theta}{\sigma \sqrt{\lambda^{\prime} V_{1} S_{1}^{-2} V_{1}^{\prime} \lambda}}}{\sqrt{\frac{y^{\prime}(1-P) y}{(n-T) \sigma^{2}}}} \mathrm{t}_{(n-T)} \text {; isto é } \\
& M=\frac{\frac{\widehat{\lambda^{\prime} \theta}-\lambda^{\prime} \theta}{\sigma \sqrt{\lambda^{\prime} V_{1} S_{1}^{-2} V_{1}^{\prime} \lambda}}}{\sqrt{\frac{(n-T) \tilde{\sigma}^{2}}{(n-T) \sigma^{2}}}}=\frac{\widehat{\lambda^{\prime} \theta}-\lambda^{\prime} \theta}{\sigma \sqrt{\lambda^{\prime} V_{1} S_{1}^{-2} V_{1}^{\prime} \lambda}} n_{(n-T)}{ }_{(n-1}
\end{aligned}
$$

Então, com base na distribuição "t", pode - se obter uma regra para estimativas por intervalo para $\lambda$ le est $^{-}$ mävel, ao nivel de confiança $1-\alpha$. 
Como os valores $\widehat{\lambda^{\prime} \theta}$ e $\hat{\sigma}$ são "observados" atra vés dos dados, adotar-se-á a notação

$$
\begin{aligned}
& t_{o b s}=\frac{\widehat{\lambda^{\prime} \theta}-\lambda^{\prime} \theta}{\sigma \cdot \sqrt{\lambda^{\prime} V_{1} s_{1}^{-2} V_{1}^{\prime \lambda}}} \\
& \text { Assim, tem-se que: }
\end{aligned}
$$

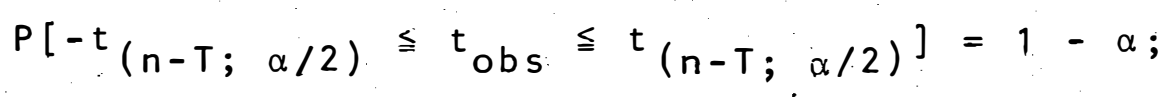

$$
\begin{aligned}
& \therefore P\left[-t(n-T ; \alpha / 2) \leqq \frac{\widehat{\lambda^{\prime} \theta}-\lambda^{\prime} \theta}{\sigma \sqrt{\lambda^{\prime} V_{1} S_{1}^{-2} V_{1}^{\prime} \lambda}} \leqq t_{(n-T ; \alpha / 2)}=1-\alpha ;\right. \\
& \text { I.C. }\left[\lambda^{\prime} \theta\right]_{1-\alpha}=\widehat{\lambda^{\prime} \theta} \pm t_{[n-T ; \alpha / 2]}\left[\hat{V}\left(\widehat{\lambda^{\prime} \theta}\right)\right]^{1 / 2}[4.24]
\end{aligned}
$$

\subsubsection{REGIÕES DE CONF I ANÇ.A}

Sejam $\lambda_{1}^{1} \theta, \quad \lambda_{2}^{\prime} \theta, \ldots, \lambda_{m}^{\prime} \theta$ funções estimáveis.

Seja também $B^{\prime} \theta=\left[\lambda_{2}, \lambda_{2}, \ldots, \lambda_{111}\right]^{\prime} \theta$ um con- 
junto de $m$ funções estimäveis e linearmente independentes.

De acordo com o teorema de Gauss-Markov

$$
\widehat{B \cdot \theta}=B \cdot \theta^{0} ;
$$

e de acordo com a propoṣição 4,

$$
B^{J} V_{1} V_{1}^{\prime}=B^{\prime}
$$

A mēdia e a variância da matriz $\widehat{B} \operatorname{lo}$ são dadas

por:

i) $E\left(\widehat{B^{\prime} \theta}\right)=E\left(B^{\prime} \theta^{0}\right)=B^{\prime} E\left(\theta^{0}\right)=B^{\prime} V_{1} V_{1}^{\prime} \theta=B^{\prime} \theta$

i i ) $V\left(\widehat{B^{\prime} \theta}\right)=V\left(B^{\prime} \theta^{0}\right)$

$$
\begin{aligned}
& =V\left(B^{\prime} V_{1} S_{1}^{-1} U_{1}^{1} y\right) \\
& =B^{\prime} V_{1} S_{1}^{-1} U_{1}^{1} V(y) U_{1} S_{1}^{-1} V_{I}^{\prime} B \\
& =B^{\prime} V_{1} S_{I}^{-2} V_{1}^{1} B \sigma^{2}
\end{aligned}
$$

portanto, tem-se que

$$
\widehat{B^{\prime} \theta} \sim \mathrm{N}_{\mathrm{m}}\left(\mathrm{B}^{\prime} \theta ; \mathrm{B}^{\prime} \mathrm{V}_{1} S_{1}^{-2} V_{1}^{\prime} B \sigma^{2}\right) .
$$

$$
\text { Seja } \mathrm{m}^{M \cdot T}=B^{\prime} V_{1} S_{I}^{-1} \text { tal que }
$$

$\operatorname{rank}\left({ }_{m} M_{T}\right)=m \leqq T=\operatorname{rank}\left(V_{1}\right)=\operatorname{rank}\left(S_{1}^{-1}\right)$; então, de acordo com a teoria das inversas generalizadas de Moore-Penrose, tem-se que $M^{+}=M^{\prime}\left(M M^{\prime}\right)^{-1}$, portanto 


$$
\begin{aligned}
& M M^{+}=1(m) \\
& \text { Tomando-se } \\
& l=\frac{M^{+}\left(B^{\prime} \theta^{0}-B^{\prime} \theta\right)}{\sigma} \text {; tem-se que: } \\
& \text { i) } E(l)=E\left[\frac{M^{+}\left(B^{\prime} \theta^{0}-B^{\prime} \theta\right)}{\sigma}\right] \\
& =\frac{1}{\sigma} M^{+} E\left(B^{\prime} \theta^{0}-B^{\prime} \theta\right) \\
& =\phi \\
& \text { i i) } \operatorname{Var}(\ell)=\operatorname{Var}\left[\frac{M^{+}\left(B^{\prime} \theta^{0}-B^{\prime} \theta\right)}{\sigma}\right] \\
& =\frac{1}{\sigma^{2} \theta} \operatorname{Var}\left[M^{+}\left(B^{\prime} \theta^{0}-B^{\prime} \theta\right)\right] \\
& =\frac{1}{\sigma^{2}} \operatorname{Var}\left[M^{+} B^{\prime} \theta^{0}\right] \\
& =\frac{1}{\sigma^{2}} M^{+1} \operatorname{Var}\left(B^{\prime} \theta^{0}\right) M^{+} \\
& =\frac{1}{\sigma^{2}} M^{+} B^{\prime} V_{1} S_{1}^{-2} V_{1} B M^{+} \sigma^{2} \\
& =M^{+} \cdot M^{\prime} M M^{+} \\
& =\left(M M^{+}\right) \cdot M M^{+}=1(m)^{\prime}(m)=1(m)
\end{aligned}
$$




$$
\begin{aligned}
& \text { Desse modo, } \\
& \ell \sim N_{m}(\phi, l(m)) \text {; tendo-se então que } \\
& \ell^{\prime} \ell \sim x^{2}(m) ; \operatorname{mas} \\
& \ell^{\prime} \ell=\frac{1}{\sigma^{2}}\left(B^{\prime} \theta^{0}-B^{\prime} \theta\right)^{\prime} M^{+} \cdot M^{+}\left(B^{\prime} \theta^{0}-B^{\prime} \theta\right) \\
& =\frac{1}{\sigma^{2}}\left(B^{\prime} \theta^{0}-B^{\prime} \theta\right)^{\prime}\left(M M^{\prime}\right)^{+}\left(B^{\prime} \theta^{0}-B^{\prime} \theta\right) \\
& =\frac{1}{\sigma^{2}}\left(B^{\prime} \theta^{0}-\dot{B}^{\prime} \theta\right)^{\prime}\left(B^{\prime} V_{1} S_{1}^{-2} V_{1}^{\prime} B\right)^{+}\left(B^{\prime} \theta^{0}-B^{\prime} \theta\right) \sim \chi^{2} r[B] \\
& \text { por outro lado; de }[4.21] \\
& \frac{1}{\sigma^{2}} y^{\prime}\left(1-P_{U_{1}}\right) y \sim x_{n-T}^{2} \\
& \text { De acordo com SEARLE (1971) } \\
& W=\frac{\frac{x^{2}[r(B)]}{r(B)}}{\frac{x^{2}(n-T)}{n-T}} \sim F \quad F[B], n-T
\end{aligned}
$$

èntão

$$
W=\frac{\frac{\left(B^{\prime} \theta^{0}-B^{\prime} \theta\right) !\left(B^{\prime} V_{l} S_{I}^{-2} B\right)^{+}\left(B^{\prime} \theta^{0}-B^{\prime} \theta\right)}{\frac{r[B] \sigma^{2}}{(n-T) y\left(1-P_{U_{1}}\right) y}}}{(n-T) \sigma^{2}} r[B], n-T
$$




$$
\begin{aligned}
& \text { Por outro lado, } \\
& \frac{(n-T) \bar{\sigma}^{2}}{\sigma^{2}} \sim x^{2}(n-T) \text { Logo, como } \\
& \bar{\sigma}^{2}=\frac{y^{\prime}\left(1-P_{U_{1}}\right) y}{n-T} \text { é um estimador não tendencioso }
\end{aligned}
$$

de $\sigma^{2}$, então

$$
W=\frac{\left.\left(B^{\prime} \theta^{0}-B^{\prime} \theta\right)\right)^{\prime}\left(B^{\prime} V_{1} S_{1}^{-2} V_{1}^{\prime} B\right)^{+}\left(B^{\prime} \theta^{0}-B^{\prime} \theta\right)}{r[B]-\frac{(n-T) \hat{\sigma}^{2}}{n-T}} \sim F \underset{r[B], n-T}{r}
$$

Portanto, um estimador por região ao nivel - de confiança 1-a, para a coleção de m funções paramétricas estimäveis $B^{\prime} \theta$ linearmente independentes, é dada por

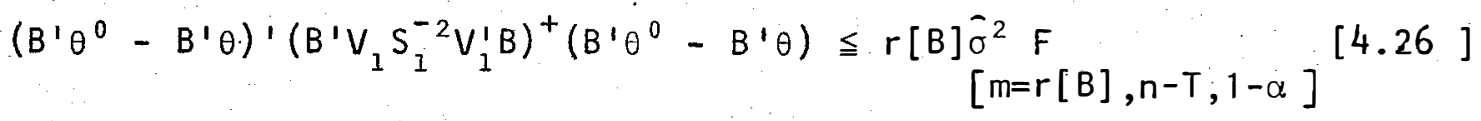

se $r[B]=2$, a região de confiança è uma elipse,

șe $r[B]>2$, a região de confiança é um elipsóide do espaço $R^{r[B]}$. 


\subsubsection{TESTES DE HIPOTESES BASEADOS EM REGIÕES DE CONF IANSCA}

Considerando-se um conjunto $B^{\prime} \theta$ de $m$ funções es timáveis linearmente independentes;

i.èe, $r(B)=m, B=\left[\lambda_{1}, \ldots, \lambda_{m}\right]$ e cada $\lambda^{\prime}{ }_{i} \theta$ é uma função estimāvel, serão testada a hipótese

$$
H_{0}: B^{\prime} \theta=\varnothing \text { vs } \quad H_{a}: B^{\prime} \theta \neq \varnothing
$$

com base em regiões de confiança.

De acordo com [4.26], a região de confiança para um conjunto de m funções estimäveis linearmente indepen dentes, ao nível $(1-\alpha)$ de probabilidade, é dada por

$$
\begin{aligned}
\left(B^{\prime} \theta^{0}-B^{\prime} \theta\right)^{\prime}\left(B^{\prime} V_{1} S_{1}^{-2} V_{1}^{\prime} B\right)^{+}\left(B^{\prime} \theta^{0}-B^{\prime} \theta\right) \leqq m^{2} \sigma_{[m, n-T ; 1-\alpha]} &
\end{aligned}
$$

$$
\text { Para se verificar } H_{0}: B^{\prime} \theta=\phi \text {, }
$$

escreve-se primeiramente a curva que contorna a região de con fiança (sob $\left.H_{0}: B^{\prime} \theta=\phi\right)$

$$
\left(B^{\prime} \theta^{0}\right)^{\prime}\left(B^{\prime} V_{1} S_{1}^{-2} V_{1}^{\prime} B\right)^{+}\left(B^{\prime} \theta^{0}\right) \leqq m \hat{\sigma}^{2} F_{\text {TABELADO }}
$$

consequentemente, a região de rejeição de $H_{0}$ será 


$$
\left(B^{\prime} \theta^{0}\right)^{\prime}\left(B^{\prime} V_{1} S_{1}^{-2} V_{1}^{\prime} B\right)^{+}\left(B^{\prime} \theta^{0}\right)>m^{-2} F_{\text {TABELADO }}^{[4.28]}
$$

ou seja,

rejeita-se $H_{0}$ : $B^{\prime} \theta=\phi$ se e só se a elipse ou o elipsóide não contèm a origem.

4.6. APLICAÇ̃̃O DOS RESULTADOS BÁSICOS NOS MODELOS LINEARES PARA UM FATGR INTE IRAMENTE CASUALIZADO

\subsubsection{INTRODUÇÃO}

Apresentam-se neste item, algumas aplicações dos resultados básicos aos modelos de um fator inteiramente casualizado, objetivando-se divulgar o uso da D.V.S. e mos trar que ela tende a ser extremamente simples, a partir do momento em que a matriz X fica bem determinada.

\subsubsection{ALGUMAS CONSIDERAÇŌES SOBRE AS MATRIZES DE HELMERT ( IEMMA, 1985)}

Tomando-se, sem perda de generalidade, uma ma triz $H_{T}$, genérica, conforme descrita a seguir, 


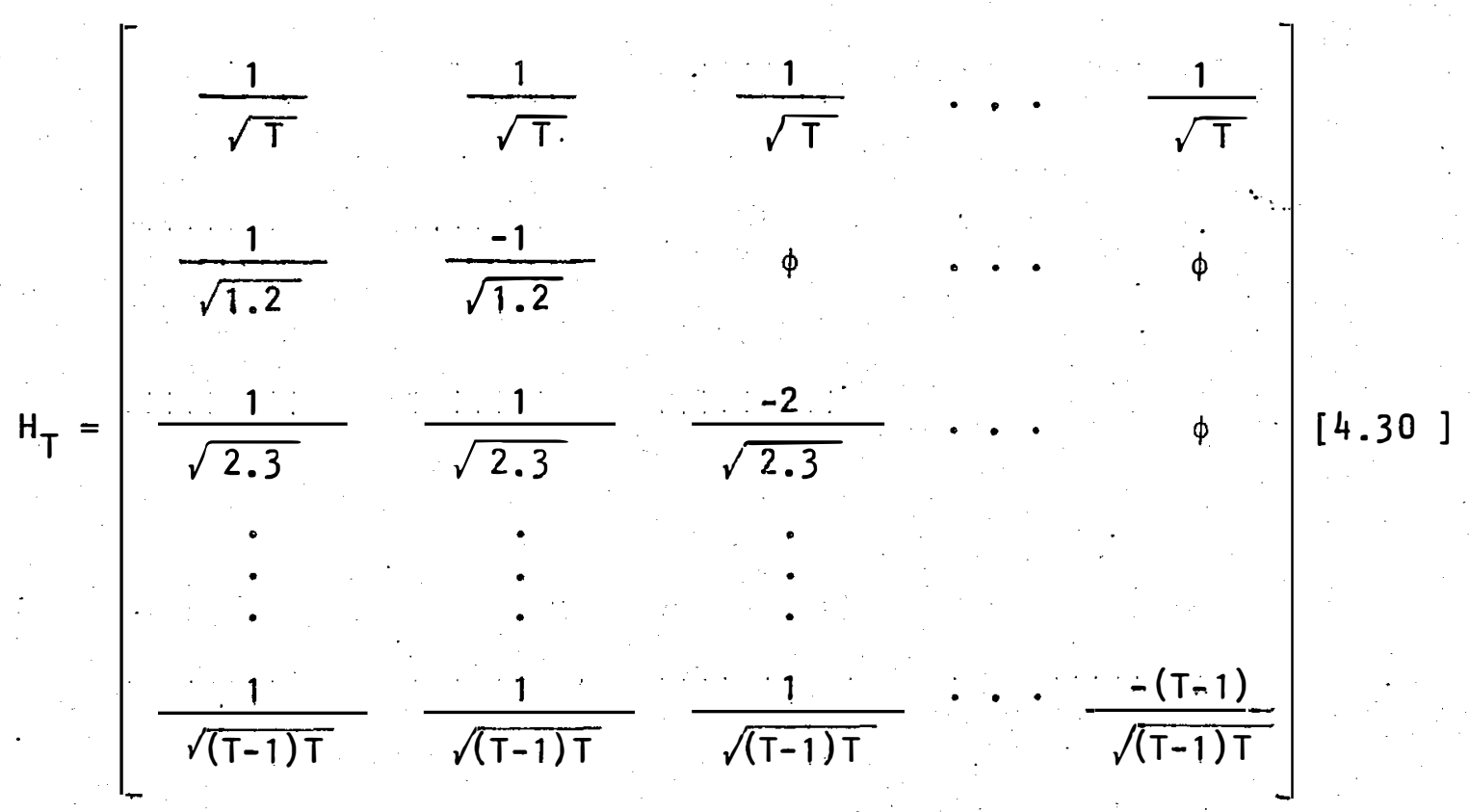

tem-se uma matriz ortogonal com lei de formação extremamente conveniente, que depende apenás de sua dimensão T.

$$
\begin{aligned}
& \text { Dada a matriz } \\
& H_{T}=\left[\begin{array}{c}
h_{T}^{h^{\prime}} \\
-H_{T}^{*}
\end{array}\right]=\left[\begin{array}{c}
-h^{\prime} \\
H^{*}
\end{array}\right]=\left[\begin{array}{r}
1^{h_{T}^{\prime}} \\
(2)_{1}^{*}{ }_{T}^{*} \\
\vdots \\
(i)_{1}^{*}{ }_{T}^{*} \\
\vdots \\
(T)_{H_{T}^{*}}^{*}
\end{array}\right]
\end{aligned}
$$

onde .$_{1}^{h}{ }_{T}$ e a primeira linha da matriz $H_{T}$ 
(j) ${ }_{1} H_{T}^{*}$ é a j-ésima linha da matriz $H_{T}$, com $j=2,3, \ldots, T$ da ortogonalidade da matriz H. IEMMA (1985) demonstra que:
a) $\mathrm{hh}^{\prime}+\mathrm{H}^{*} \mathrm{H}^{*}=1(\mathrm{~T})$
$[4.31]$
b) $h^{\prime} h=1$
[4.32.]
c) $h^{1} H^{* 1}=\phi$ e $H^{*} h=\phi$
[4.33]
d) $H * H * 1=1(T-1)$
[4.34]

e) $H^{* 1} H^{*}=\frac{1}{T}\left[\begin{array}{ccccc}T-1 & -1 & -1 & \ldots & -1 \\ -1 & T-1 & -1 & \ldots & -1 \\ -1 & -1 & T-1 & \ldots & -1 \\ \vdots & \vdots & \vdots & \ldots & \vdots \\ -1 & -1 & -1 & \ldots & T-1\end{array}\right]\left[\begin{array}{lll}4.35 & \end{array}\right]$ 
.60 .

\subsubsection{O ALGORITMO}

\subsubsection{A DECOMPOSIÇÃO PELO SEU VALOR SINGULAR DA MATRIZ ${ }_{n} X_{P}$ DO DELINEAMENTO COM UM FATOR INTEIRAMENTE CASUALIZADO}

No delineamento com $(p-1)$ tratamentos e R repetições, a matriz de planejamento é da forma:

$$
\mathrm{n}_{\mathrm{P}}=\left[\begin{array}{ccccc}
1 & 1 & 0 & \cdots & 0 \\
1 & 1 & 0 & \cdots & 0 \\
\vdots & \vdots & \vdots & & \vdots \\
1 & 1 & 0 & \cdots & 0 \\
1 & 0 & 1 & \cdots & 0 \\
1 & 0 & 1 & \cdots & 0 \\
\vdots & \vdots & \vdots & & \vdots \\
1 & 0 & 1 & \cdots & 0 \\
\vdots & \vdots & \vdots & & \vdots \\
1 & 0 & 0 & \cdots & 1 \\
1 & 0 & 0 & \cdots & 1 \\
\vdots & \vdots & \vdots & & \vdots \\
1 & 0 & 0 & \cdots & 1
\end{array}\right]_{\mathrm{P}}
$$

onde $\operatorname{posto}(X)=p-1=T$

$[4.37]$

$\mathrm{n}=\mathrm{R} \times \mathrm{T}$

$[4.38]$ 
4.6.2.1.1. Os autovalores não nulos $s_{1}^{2}, s_{2}^{2}, \ldots, s_{T}^{2}$ de $X X .^{\prime} \quad\left(X^{\prime} X\right.$, respectivamente)

Proposição 5. No modelo do presente estudo, a matriz XX' é gerada pelo produto de Kronecker de matrizes $\underline{u}$ niformes.

Prova:

Se jam. as natrizes uniformes

$E_{R}=\left[\begin{array}{cccc}1 & 1 & \cdots & 1 \\ 1 & 1 & \cdots & 1 \\ \vdots & \vdots & & \vdots \\ 1 & 1 & & 1\end{array}\right]_{R}$

$A_{T}=\left[\begin{array}{cccc}2 & 1 & \cdots & 1 \\ 1 & 2 & \cdots & 1 \\ \vdots & \vdots & & \vdots \\ 1 & 1 & \cdots & 2\end{array}\right]_{T}$ 
então

$A_{T} \circlearrowright E_{R}=\left[\begin{array}{cccccc}2 E_{R} & E_{R} & E_{R} & \cdots & E_{R} \\ E_{R} & 2 E_{R} & E_{R} & \cdots & E_{R} \\ E_{R} & E_{R} & 2 E_{R} & \cdots & E_{R} \\ \vdots & \vdots & \vdots & & \vdots \\ E_{R} & E_{R} & E_{R} & \cdots & 2 E_{R}\end{array}\right]_{n=(R . T)}=x x^{\prime}$

concluindo a demonstração.

De acordo com IEMMA (1982), os autovalores das matrizes uniformes $A_{T}$ e $E_{R}$ são dados por:

a) Autovalores de $A_{T}: \alpha_{1}, \alpha_{2}, \ldots, \alpha_{T}$, onde

$$
\begin{aligned}
& \alpha_{1}=2+(T-1)=T+1 \\
& \left.\begin{array}{l}
\alpha_{2}=1 \\
\vdots \\
\alpha_{T}=1
\end{array}\right\} \text { multiplicidade }(T-1)
\end{aligned}
$$

b) Autovalores de $E_{R}: \beta_{1}, \beta_{2}, \ldots, \beta_{R}$ onde

$$
\beta_{2}=1+(R-1)=R
$$




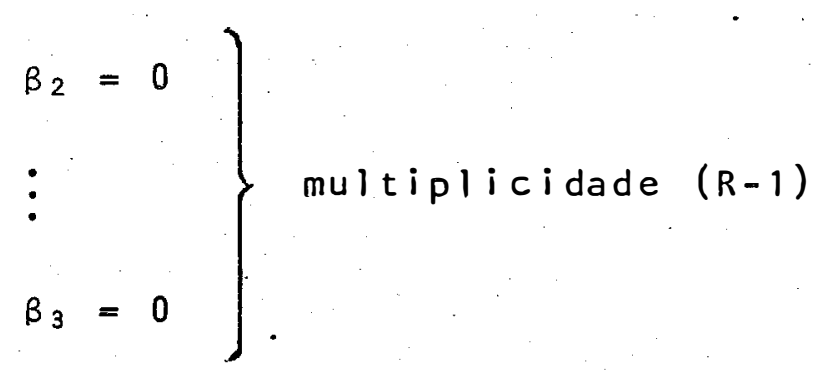

IEMMA (1987a) demonstra que os autovalores não nulos de $A_{T}$ (x) $E_{R}$ serão:

$$
\begin{aligned}
& s_{1}^{2}=\alpha_{1} \beta_{1}=(T+1) R \\
& s_{2}^{2}=\alpha_{2} \beta_{1}=R \\
& \vdots \\
& s_{T}^{2}=\alpha_{T} \beta_{1}=R
\end{aligned}
$$

portanto,

$$
S_{1}=\left[\begin{array}{ccccc}
\sqrt{(T+1) R} & \phi & \phi & \cdots & \phi \\
\phi & \sqrt{R} & \phi & \cdots & \phi \\
\phi & \phi & \sqrt{R} & \cdots & \phi \\
\vdots & \vdots & \vdots & & \\
\phi & \phi & \phi & \cdots & \sqrt{R}
\end{array}\right]_{T}
$$


4.6.2.1.2. Os autovetores normalizados de $X X^{\prime}$, correspondentes aos autovalores não nulos $s_{1}^{2}, s_{2}^{2}, \ldots, s_{T}^{2}$

De acordo com IEMMA (1985), sendo $A_{T}$ uma matriz uniforme de ordem $T$, existe uma matriz ortogonal $H_{T}$ tal que

$$
\left.H^{\prime} A H=D \text { (decomposição espectral de } A_{T}\right)
$$

onde

H è uma matriz de Helmert, cujas linhas são os vetores característicos normalizados, associados aos au tovalores de A;

D é a matriz diagonal que exibe as raízes caracterís ticas de $A$.

Sendo $A_{T}=\left[\begin{array}{cccc}2 & 1 & \ldots & 1 \\ 1 & 2 & \ldots & 1 \\ \vdots & \vdots & & \\ 1 & 1 & \ldots & 2\end{array}\right]$,

os autovetores associados aos autovalores não nulos $\alpha_{1}, \alpha_{2}, \ldots, \alpha_{T}$, serão: 
$1_{T}^{\prime}=\left(\frac{1}{\sqrt{T}} \frac{1}{\sqrt{T}} \cdot \frac{1}{\sqrt{T}} \cdots \frac{1}{\sqrt{T}}\right)$

${ }_{1}^{(2)} H_{T}^{*}=\left(\frac{1}{\sqrt{1.2}} \frac{-1}{\sqrt{1.2}} \phi \quad \phi \quad \ldots \quad \phi\right)$

(3) ${ }_{1} H_{T}^{*}=\left[\frac{1}{\sqrt{2.3}} \frac{1}{\sqrt{2.3}} \frac{-2}{\sqrt{2.3}} \quad \phi \quad \cdots \phi\right]$

$\vdots$

(T) ${ }_{1} H_{T}^{*}=\left[\frac{1}{\sqrt{(T-1) T}} \frac{1}{\sqrt{(T-1) T}} \frac{1}{\sqrt{(T-1) T}} \cdots \frac{-(T-1)}{\sqrt{(T-1) T}}\right]$,

e para matriz uniforme $E_{R}=\left[\begin{array}{cccc}1 & 1 & \cdots & 1 \\ 1 & 1 & \cdots & 1 \\ \vdots & \vdots & & \vdots \\ 1 & 1 & & 1\end{array}\right]$

o autovetor associado ao autovalor não nulo $\beta_{1}=R$ será:

$$
{ }_{1} h_{R}=\left(\frac{1}{\sqrt{R}} \frac{1}{\sqrt{R}} \cdots \frac{1}{\sqrt{R}}\right)
$$

Proposição 6. Os autovetores normalizados de XX', relativos aos autovalores não nulos $s_{l}^{2}, s_{2}^{2}, \ldots, s_{T}^{2}$ são dados por: 


$$
\begin{aligned}
& u_{i}=1^{h^{\prime}} T \otimes 1^{h^{\prime}} \cdot R \quad e \\
& u_{j}^{\prime}=(j){ }_{1} H_{T}^{*} \otimes \quad{ }_{1}{ }^{\prime}{ }_{R} \operatorname{com} j=2,3, \ldots, T \\
& \text { portanto, }
\end{aligned}
$$

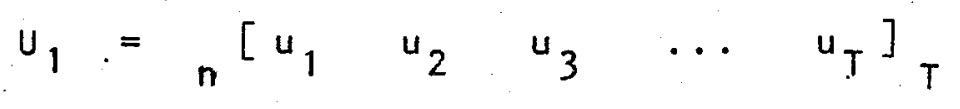

$$
\begin{aligned}
& \text { ou seja, }
\end{aligned}
$$




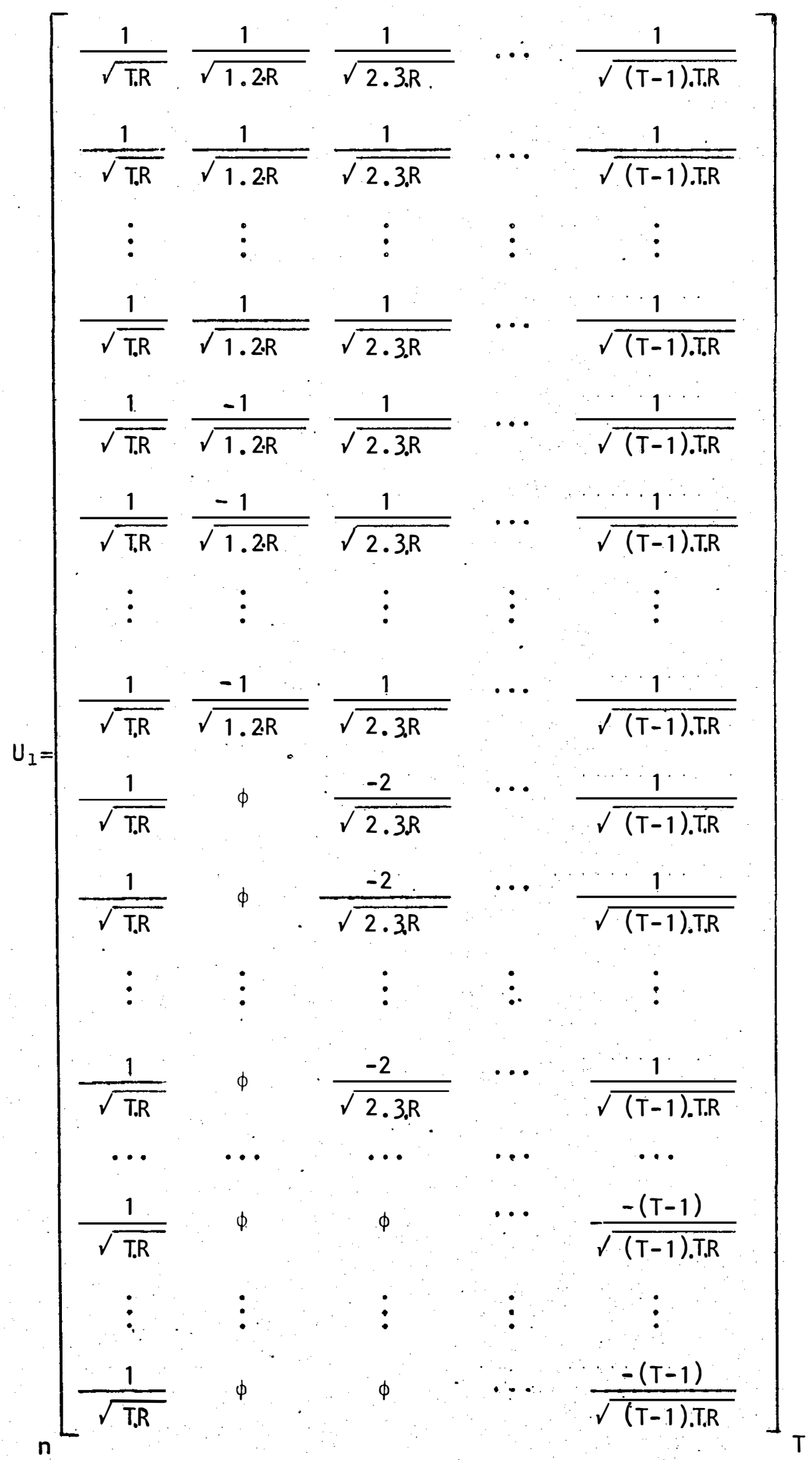


deixando-se $\frac{1}{\sqrt{R}}$ em evidência,

$$
\begin{aligned}
& \begin{array}{ccccc}
\frac{1}{\sqrt{T}} & \frac{1}{\sqrt{2}} & \frac{1}{\sqrt{6}} & \cdots & \frac{1}{\sqrt{T(T-1)}} \\
\vdots & \vdots & \vdots & & \vdots \\
\frac{1}{\sqrt{T}} & \frac{1}{\sqrt{2}} & \frac{1}{\sqrt{6}} & \cdots & \frac{1}{\sqrt{T(T-1)}}
\end{array} \\
& \frac{1}{\sqrt{T}} \frac{-1}{\sqrt{2}} \cdot \frac{1}{\sqrt{6}} \cdots \frac{1}{\sqrt{T(T-1)}} \\
& \begin{array}{llll}
\vdots & \vdots & \vdots & \vdots
\end{array} \\
& \frac{1}{\sqrt{T}} \quad \frac{-1}{\sqrt{2}} \quad \frac{1}{\sqrt{6}} \cdots \frac{1}{\sqrt{T(T-1)}} \\
& U_{1}=\frac{1}{\sqrt{R}} \quad \frac{1}{\sqrt{T}} \quad \phi \quad \frac{-2}{\sqrt{6}} \cdots \frac{1}{\sqrt{T(T-1)}} \\
& \vdots \quad \vdots \quad \vdots \quad \vdots \\
& \frac{1}{\sqrt{T}} \quad \phi \quad \frac{-2}{\sqrt{6}} \cdots \frac{1}{\sqrt{T(T-1)}} \\
& \begin{array}{lllll}
\ldots & \ldots & \ldots & \ldots & \ldots
\end{array} \\
& \begin{array}{llllll} 
& \ldots & \ldots & \ldots & \ldots &
\end{array} \\
& \frac{1}{\sqrt{T}} \cdot \phi \quad \phi \quad \cdots \frac{-(T-1)}{\sqrt{T(T-1)}} \\
& \vdots \quad \vdots \quad \vdots \quad \text { - } \quad \vdots \\
& \frac{1}{\sqrt{T}} \quad \phi \quad \phi \quad \cdots \frac{-(T-1)}{\sqrt{T(T-1)}} \\
& n
\end{aligned}
$$


como

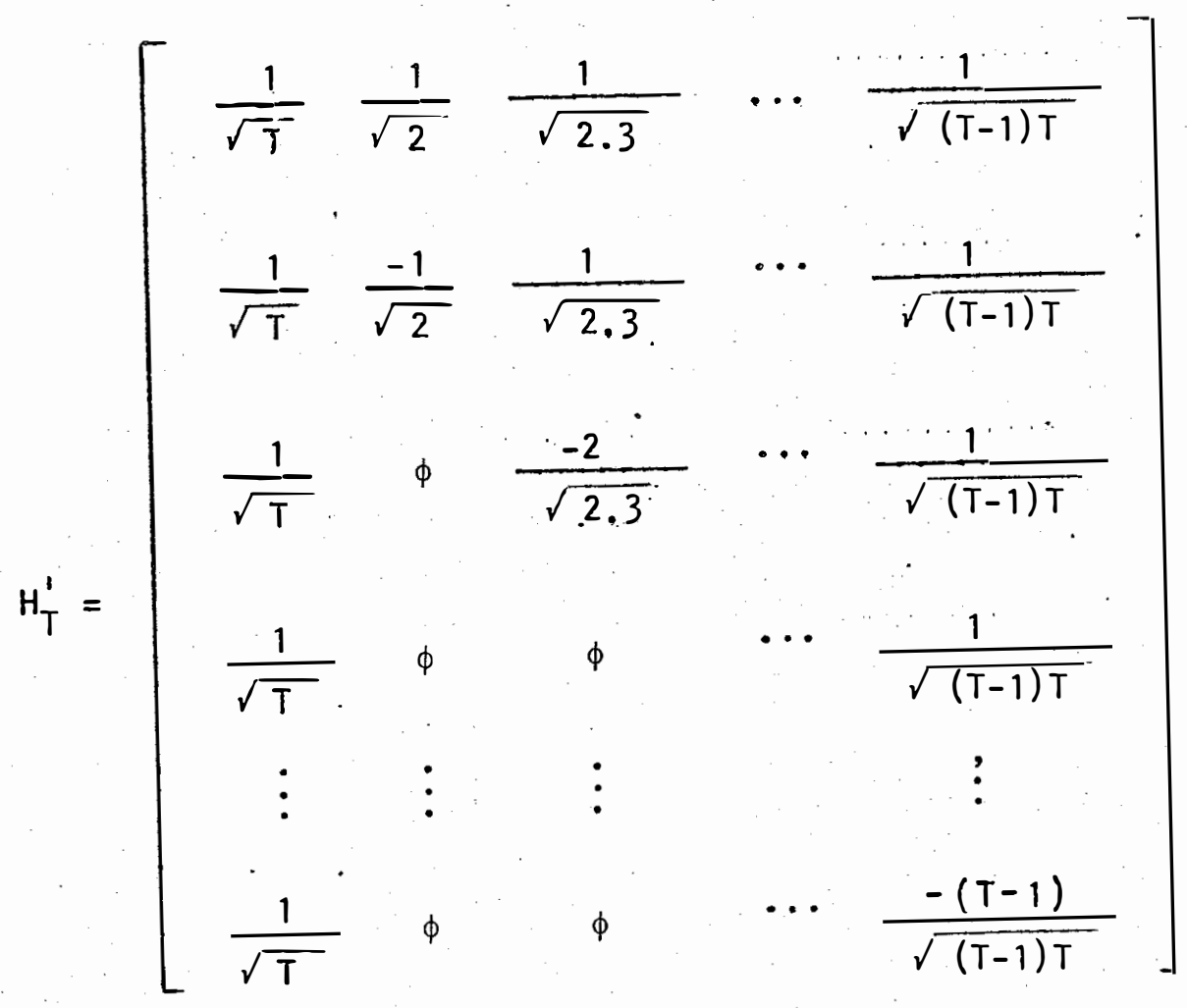

então tem-se que

$$
U_{I}=\frac{1}{\sqrt{R}}\left[H_{T}^{\prime} \Leftrightarrow R_{1}^{E_{1}}\right]
$$

onde

$$
R_{1}^{E_{1}}=\left[\begin{array}{c}
1 \\
1 \\
\vdots \\
1
\end{array}\right]
$$


4.6.2.1.3. Os autovetores normalizados de $x ! X$, correspondentes aos autovalores não nulos $\mathrm{s}_{1}^{2}, \mathrm{~s}_{2}^{2}, \ldots, \mathrm{s}_{\mathrm{T}}^{2}$

$$
\text { os autovetores } v_{1}, v_{2}, \ldots, v_{T} ; \text { relativos }
$$

aos autovalores positivos $s_{1}^{2}, s_{2}^{2}, \ldots, s_{T}^{2}$ de $x^{\prime} x$, são dados por:

$$
v_{i}=\frac{1}{s_{i}} \times u_{i}
$$

ou seja

$$
v_{1}=s_{1}^{-1} x^{\prime} U_{I}=\underbrace{}_{P}\left[\begin{array}{llll}
v_{1} & v_{2} & \cdots & v_{T}
\end{array}\right]_{T}
$$

$$
\begin{aligned}
& \text { assim sendo, tem-se que: } \\
& v_{1}=\frac{1}{\sqrt{(T+1) R}}\left[\begin{array}{ccccccccccccc}
1 & 1 & \ldots & 1 & 1 & 1 & \ldots & 1 & \ldots & 1 & 1 & \ldots & 1 \\
1 & 1 & \ldots & 1 & 0 & 0 & \ldots & 0 & \ldots & 0 & 0 & \ldots & 0 \\
0 & 0 & \ldots & 0 & 1 & 1 & \ldots & 1 & \ldots & 0 & 0 & \ldots & 0 \\
\vdots & \vdots & & \vdots & \vdots & \vdots & & \vdots & & \vdots & \vdots & & \vdots \\
0 & 0 & \ldots & 0 & 0 & 0 & \ldots & 0 & \ldots & 1 & 1 & \ldots & 1
\end{array}\right]\left[\begin{array}{c}
\frac{1}{\sqrt{\mathrm{TR}}} \\
\frac{1}{\sqrt{\mathrm{TR}}} \\
\frac{1}{\sqrt{\mathrm{TR}}} \\
\frac{1}{\sqrt{\mathrm{TR}}} \\
\vdots \\
\frac{1}{\sqrt{\mathrm{TR}}}
\end{array}\right]
\end{aligned}
$$


efetuando-se o produto matricial,

$$
v_{1}^{\prime}=\left(\frac{T}{\sqrt{T(T+1)}} \frac{1}{\sqrt{T(T+1)}} \cdot \frac{1}{\sqrt{T(T+1)}} \cdots \frac{1}{\sqrt{T(T+1)}}\right)
$$

de maneira análoga,

$$
\begin{aligned}
& v_{2}^{\prime}=\left(\phi \frac{1}{\sqrt{2}} \frac{-1}{\sqrt{2}} \cdots \phi \quad \ldots . \phi\right)
\end{aligned}
$$

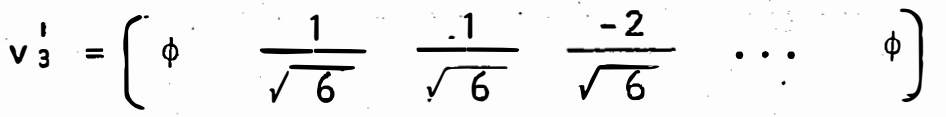

$$
\begin{aligned}
& v_{4}^{\prime}=\left[\phi \cdot \frac{1}{\sqrt{12}} \frac{1}{\sqrt{12}} \frac{1}{\sqrt{12}} \frac{-3}{\sqrt{12}} \phi \ldots \ldots \phi\right) \\
& \vdots \\
& v_{T}^{\prime}=\left(\phi \frac{1}{\sqrt{T(T-1)}} \frac{1}{\sqrt{T(T-1)}} \cdots \frac{1}{\sqrt{T(T-1)}} \frac{-(T-1)}{\sqrt{T(T-1)}}\right)
\end{aligned}
$$$$
\text { Portanto, a matriz }{ }_{P}^{V_{I}} \text { é dado por: }
$$

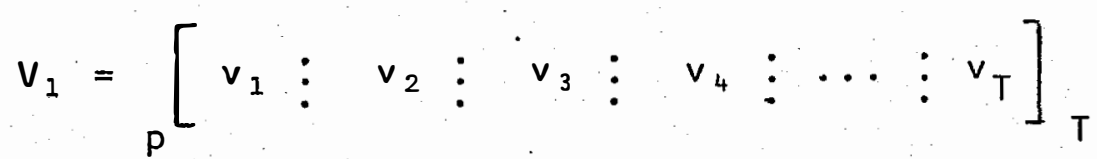

ou seja, 


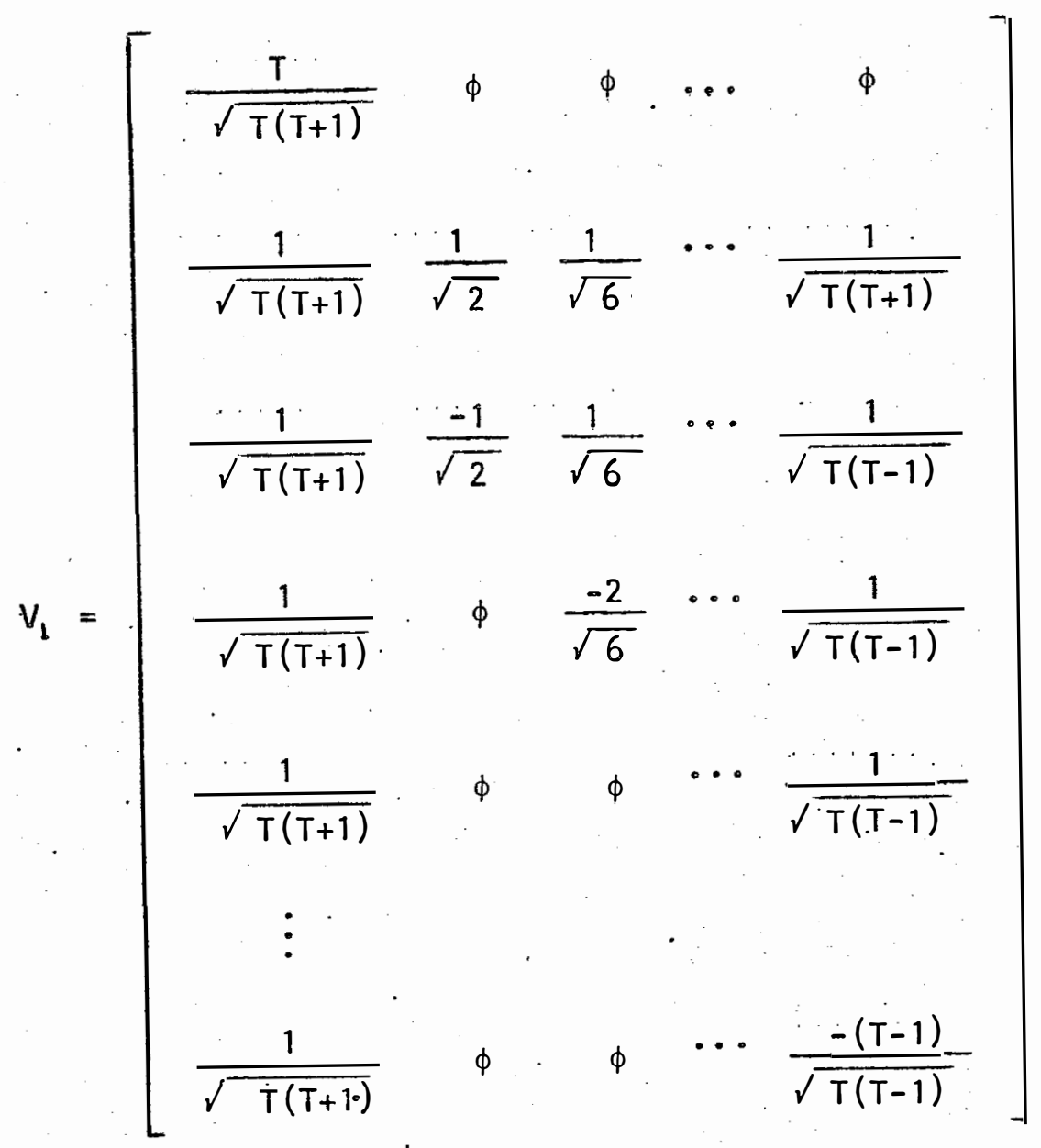

Fato: A lei de formação dos autovalores de $X X^{\prime}$ e $X^{\prime} X$ e dos res pectivos autovetores normalizados é extremamente simples pelo enfoque proposto neste estudo, estando em fun ção do nümero de tratamentos $T=P-1$ e o número $R$ de re petiçōes da matriz do del ineamento $n^{X_{p}}$.

$$
\text { Verifica-se a seguir que } U_{1} S_{1} V_{1}^{\prime}={ }_{n} X_{p} \text {, o que }
$$
valida o algoritmo. 
.73 .

$4 \cdot 6 \cdot 2 \cdot 1 \cdot 4$. Verificando $U_{I} S_{I} V_{I}^{\prime}={ }_{n} X_{p}$

De acordo com os resultados [4.39] e [4.41],

tem-se

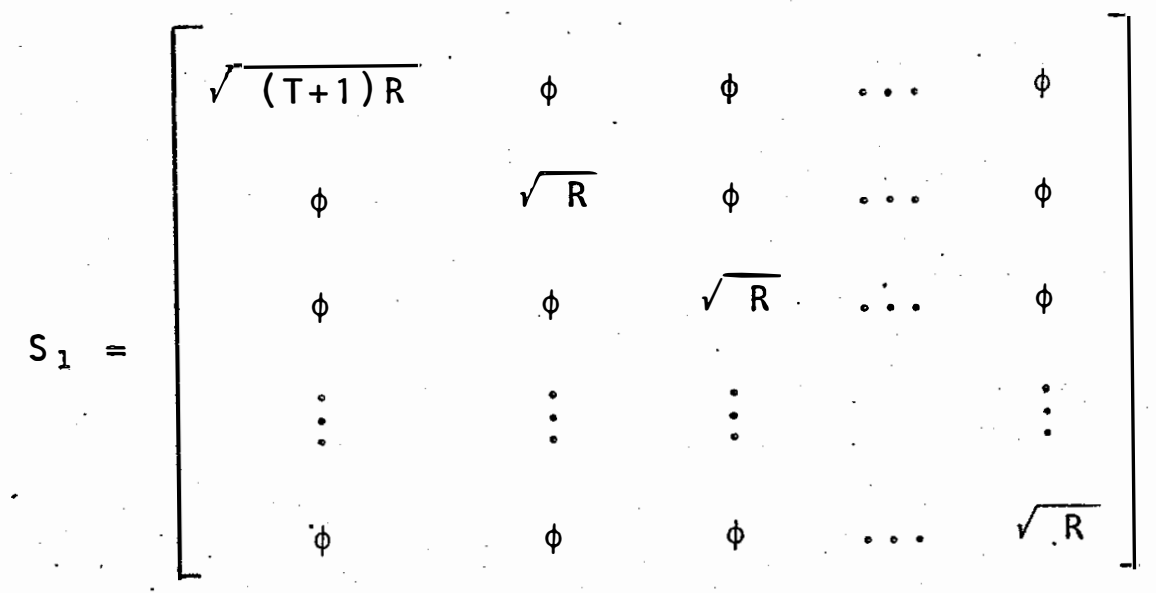

e

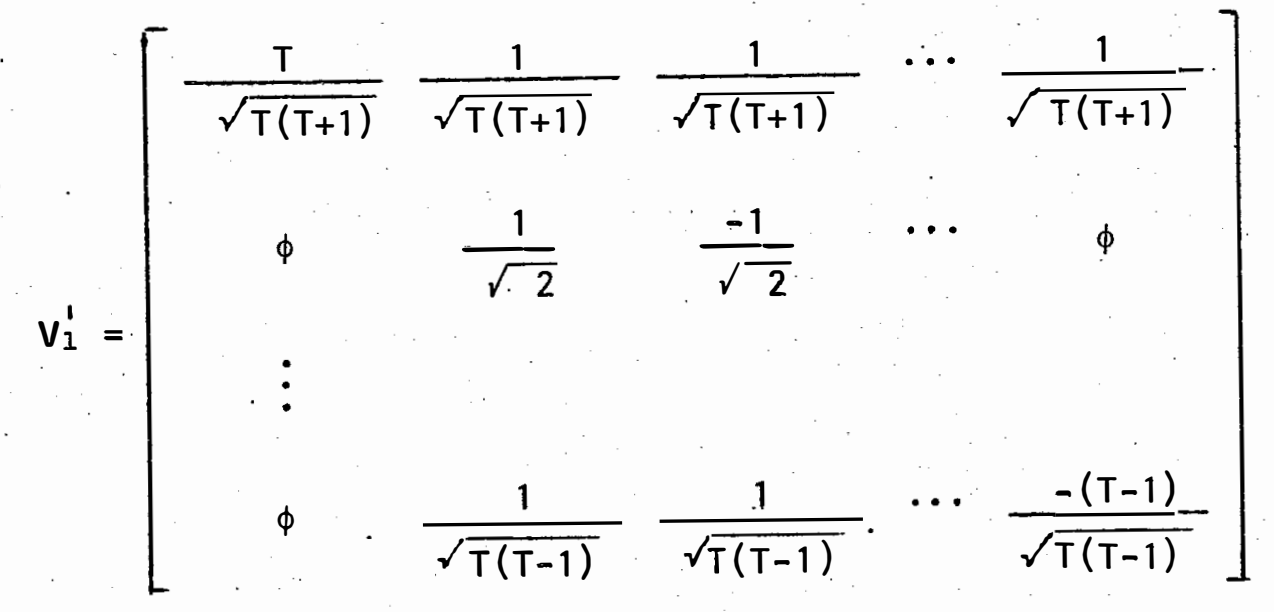

[4.42]

e o produto $S_{1} V_{1}^{\prime}$ é dado por: 


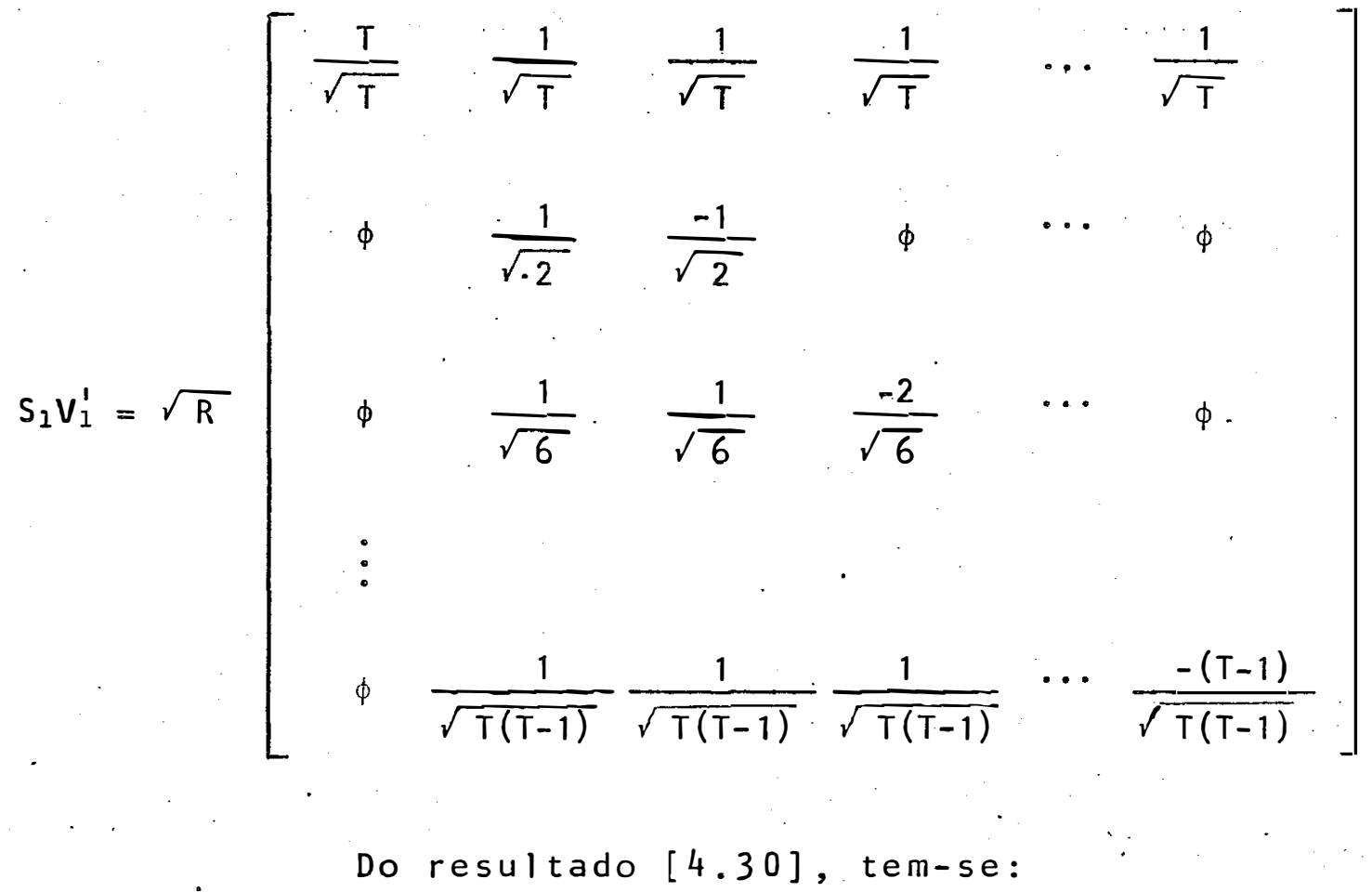

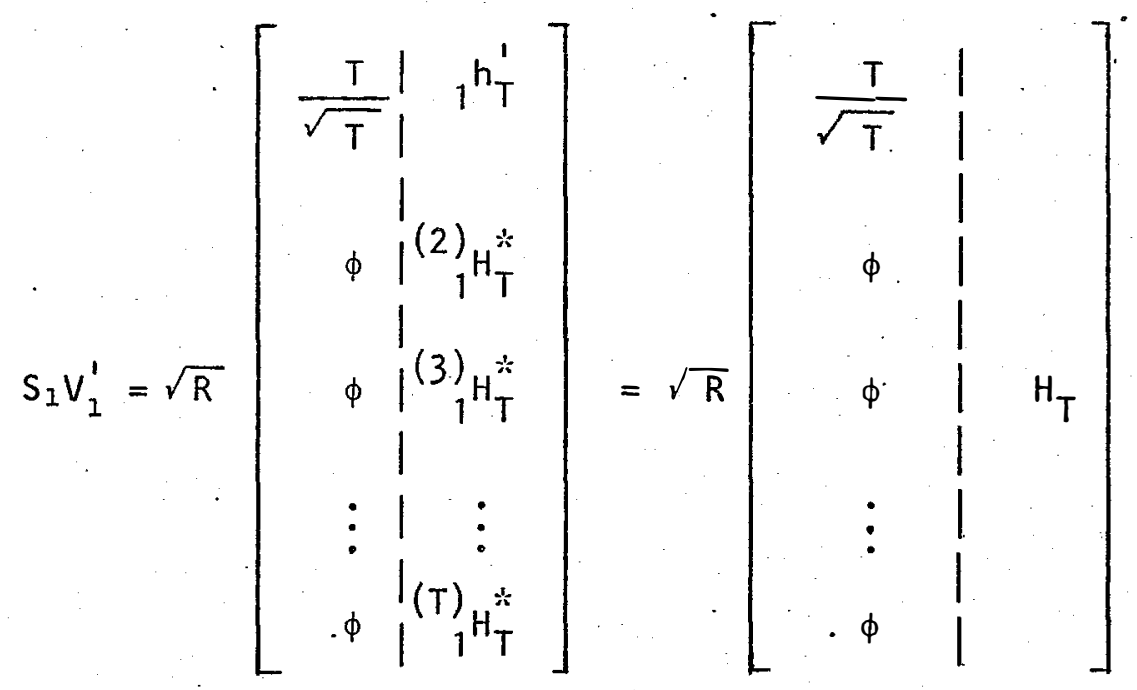

onde $H_{T}$ é a matriz de Helmert.

Dos resultados [4.40] e [4.43] segue-se que: 


$$
\begin{aligned}
& U_{1} S_{7} V_{1}^{\prime}=\frac{1}{\sqrt{R}}\left(H_{T}^{\prime}(x) E_{R}\right) \sqrt{R}\left[\begin{array}{cc}
\frac{T}{\sqrt{T}} \mid & \\
\phi & 1 \\
\phi & 1 \\
& \vdots \\
\vdots & 1
\end{array}\right] \\
& =\left(H_{T}^{\prime}\left(R_{1}^{E_{1}}\right)\left[\begin{array}{ccc}
\frac{T}{\sqrt{T}} & 1 \\
\phi & 1 & H_{T} \\
\phi & 1 & \\
\vdots & 1 & \\
\phi & 1
\end{array}\right]\right.
\end{aligned}
$$

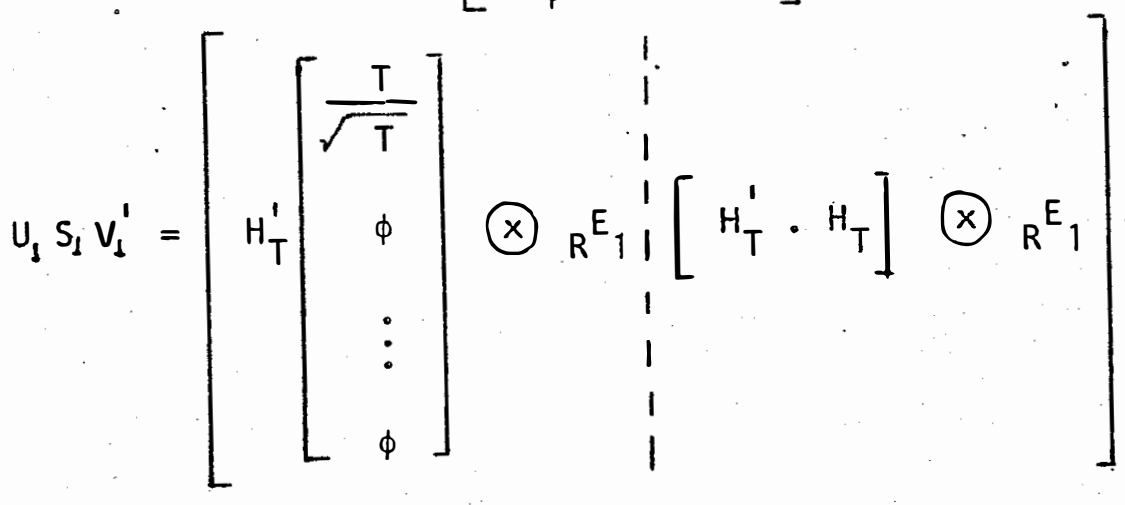

$$
\begin{aligned}
& \text { de acordo } \operatorname{com}[4.31], H_{T}^{\prime}, H_{T}=I_{T} \text {, então } \\
& U_{1} S_{1} V_{1}^{\prime}=\left[\begin{array}{l|llllllll}
1 & 1 & 1 & 0 & 0 & 0 & 0 & 0 & 0 \\
1 & 1 & 0 & 1 & 0 & 0 & 0 & 0 & 0 \\
1 & 0 & 0 & 1 & 0 & 0 & 0 & 0 \\
1 & 0 & 0 & 0 & 1 & 0 & 0 & 0 \\
1 & 0 & 0 & 0 & 0 & 1 & 0 & 0 \\
1 & 0 & 0 & 0 & 0 & 0 & 1 & 0 \\
1 & 0 & 0 & 0 & 0 & 0 & 0 & 1
\end{array}\right] \times{ }_{R} E_{1}
\end{aligned}
$$


donde se conclui

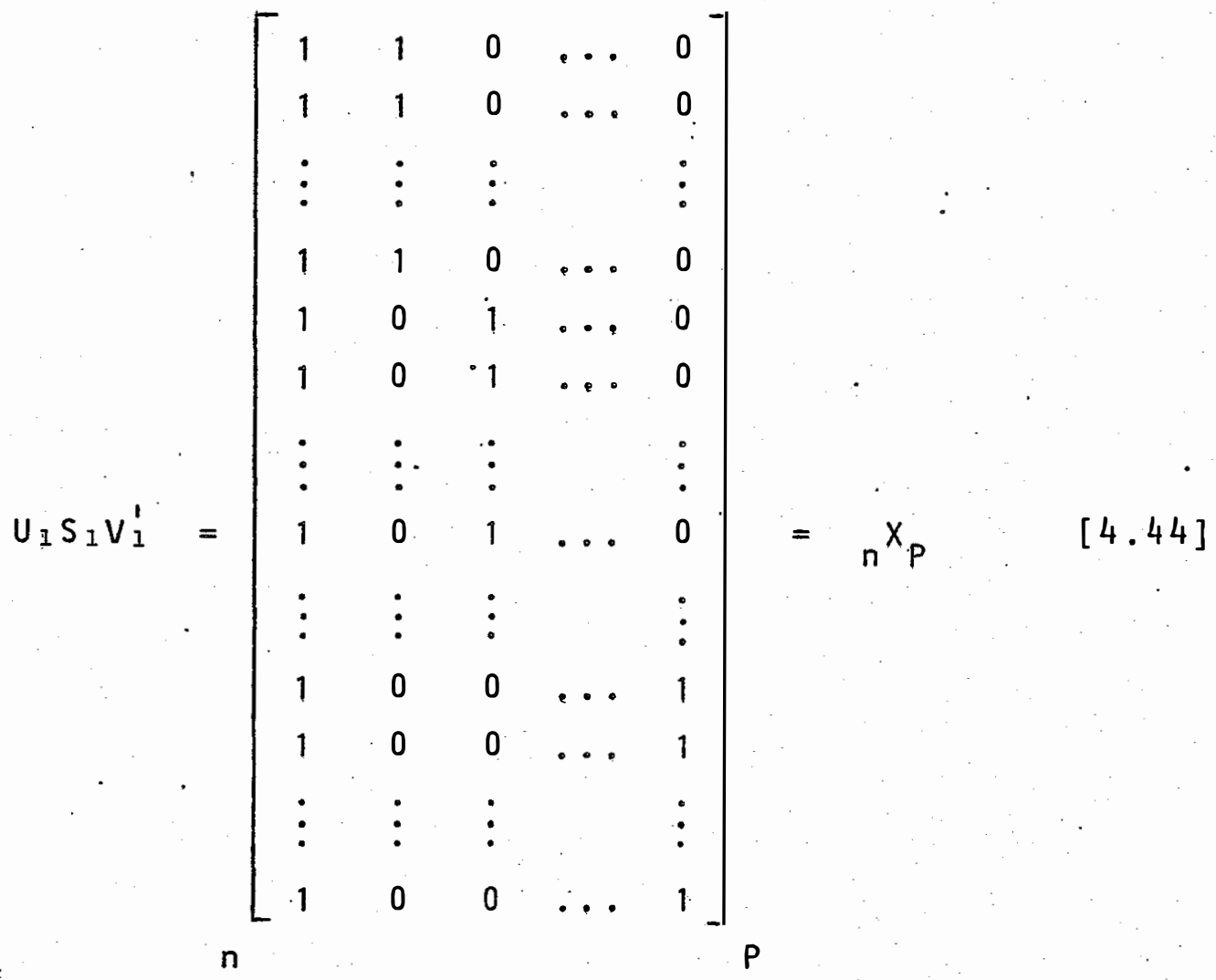

finalizando a demonstração.

$$
4 \cdot 6.2,1.5 \text {. Resumo }
$$

Dada a matriz do delineamento do modelo experimental inteiramente casualizado com $T$ tratamentos e $R$ repe tições, de acordo com o processo aqui proposto, que será denominado de "Algoritmo ASSIZ CARVALHO/IEMMA". sua D.V.S., da da por ${ }_{n} X_{P}=U_{1} s_{1} V_{1}^{\prime}$, será: 
$U_{1}=\frac{1}{\sqrt{R}}{ }_{n}\left[H_{T}^{\prime} \otimes R^{E_{1}}\right]_{T} ;$ de acordo com [4.40] $\mathrm{S}_{1}=\left[\begin{array}{ccccc}\sqrt{(T+1) R} & \phi & \phi & \cdots & \phi \\ \phi & \sqrt{R} & \phi & \cdots & \phi \\ \phi & \phi & \sqrt{R} & \cdots & \phi \\ \vdots & \vdots & \vdots & & \vdots \\ \phi & \phi & \phi & \cdots & \sqrt{R}\end{array}\right]_{T}$; de acordo com [4.39] $V_{1}^{\prime}=\left[\begin{array}{ccccc}\frac{T}{\sqrt{T(T+1)}} & \phi & \phi & \cdots & \phi \\ \frac{1}{\sqrt{T(T+1)}} & \frac{1}{\sqrt{1.2}} & \frac{1}{\sqrt{2.3}} & \cdots & \frac{1}{\sqrt{(T-1) T}} \\ \frac{1}{\sqrt{T(T+1)}} & \frac{-1}{\sqrt{1.2}} & \frac{1}{\sqrt{2.3}} & \cdots & \frac{1}{\sqrt{(T-1) T}} \\ \vdots & \vdots & \vdots & & \vdots \\ \frac{1}{\sqrt{T(T+1)}} & \phi & \phi & \cdots & \frac{-(T-1)}{\sqrt{(T-1) T}}\end{array}\right]$ d de acordo com [4.42]

4:6:3. ALGUNS RESULTADOS ÚTEIS

4.6.3.1. O PROJETOR ORTOGONAL EM C $(X)$

o projetor ortogonal em $c(x)$ é dado por $P_{X}=U_{1} U_{1}^{\prime}=P_{U_{1}}$. 
.78.

De acordo com o resultado [4.26], tem-se:

$$
\begin{aligned}
& P_{U_{1}}=\frac{1}{\sqrt{R}}\left[H_{T}^{\prime} \otimes R_{R} E_{1}\right]\left[\begin{array}{lll}
{ }_{1} E_{R} & \otimes & H_{T}
\end{array}\right] \frac{1}{\sqrt{R}} \\
& =\frac{1}{R} H_{T}^{\prime} \cdot H_{T} \otimes{ }_{R} E_{R} ; \text { mas } H_{T}^{\prime} H_{T}=I_{(T)} \text { : } \\
& =\frac{1}{R}{ }^{\prime}(T) \otimes R_{R} E_{R}
\end{aligned}
$$

[4.45]

4.6.3.2. O PROJETOR ORTOGONAL EM $C\left(X^{\prime}\right)$

De [4.41] tem-se que:

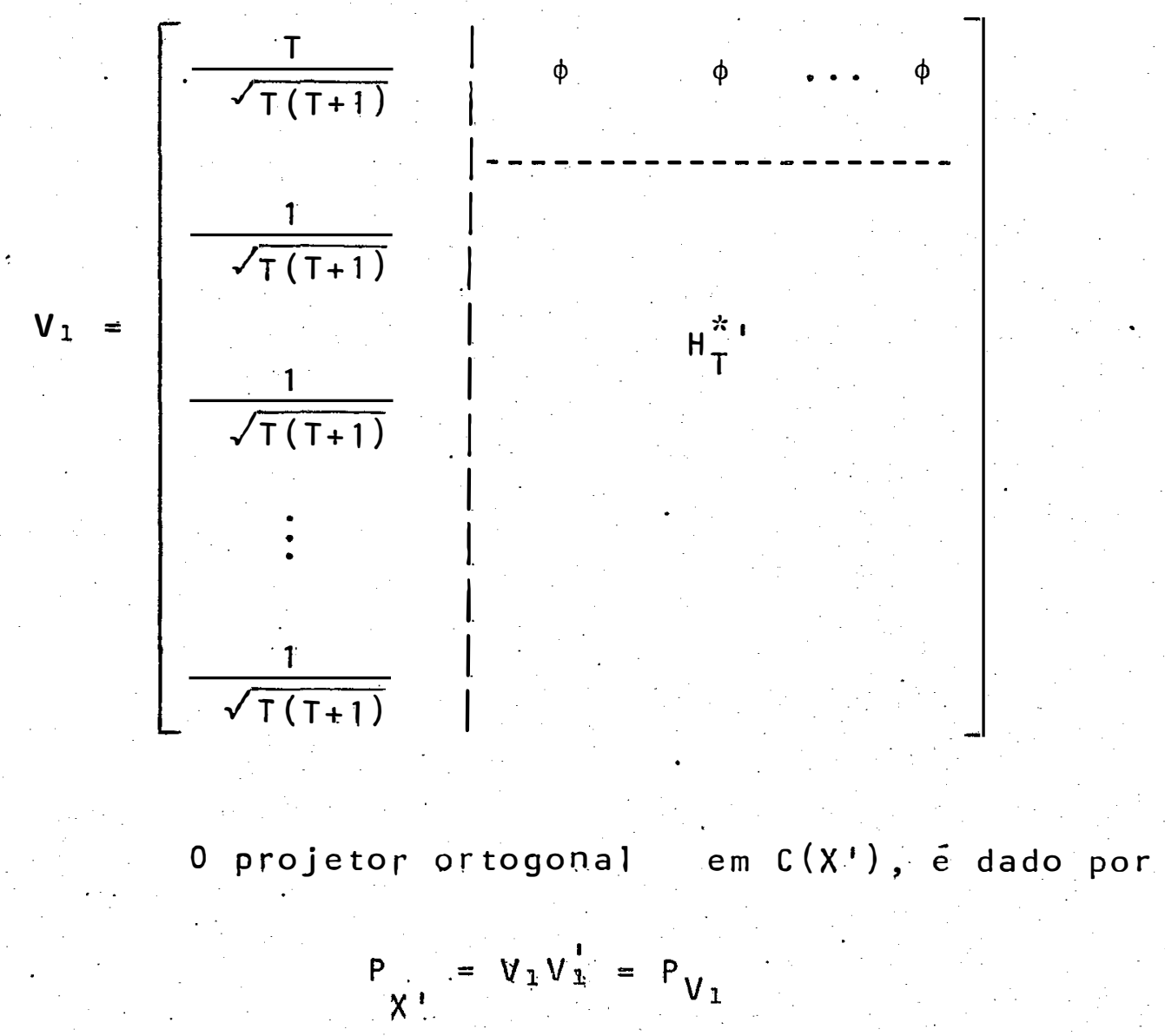


De acordo com o resultado [4.41] viu-se que

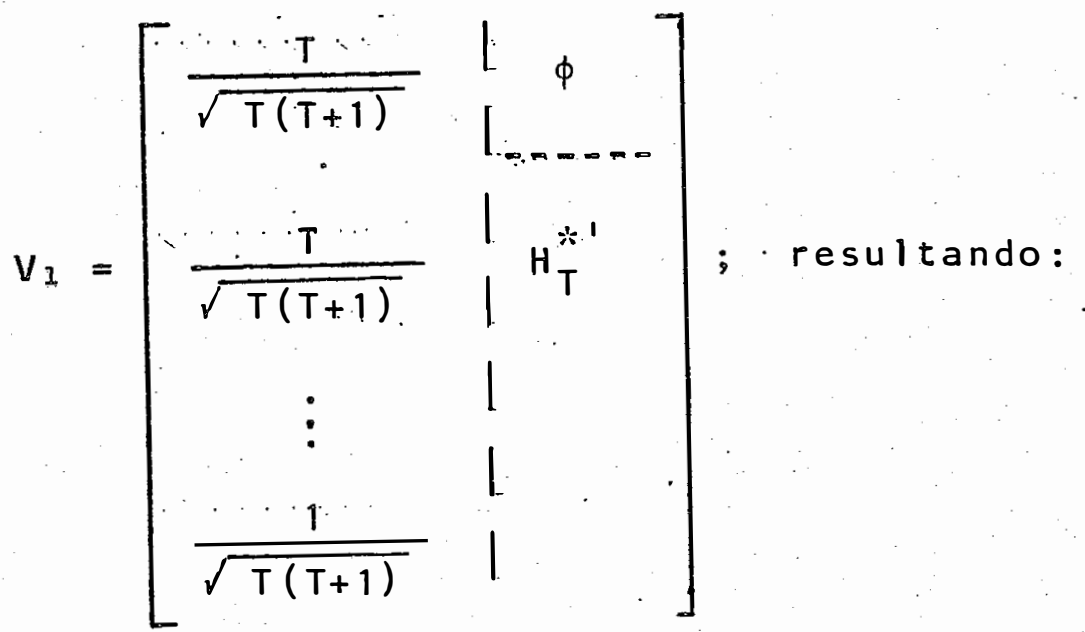

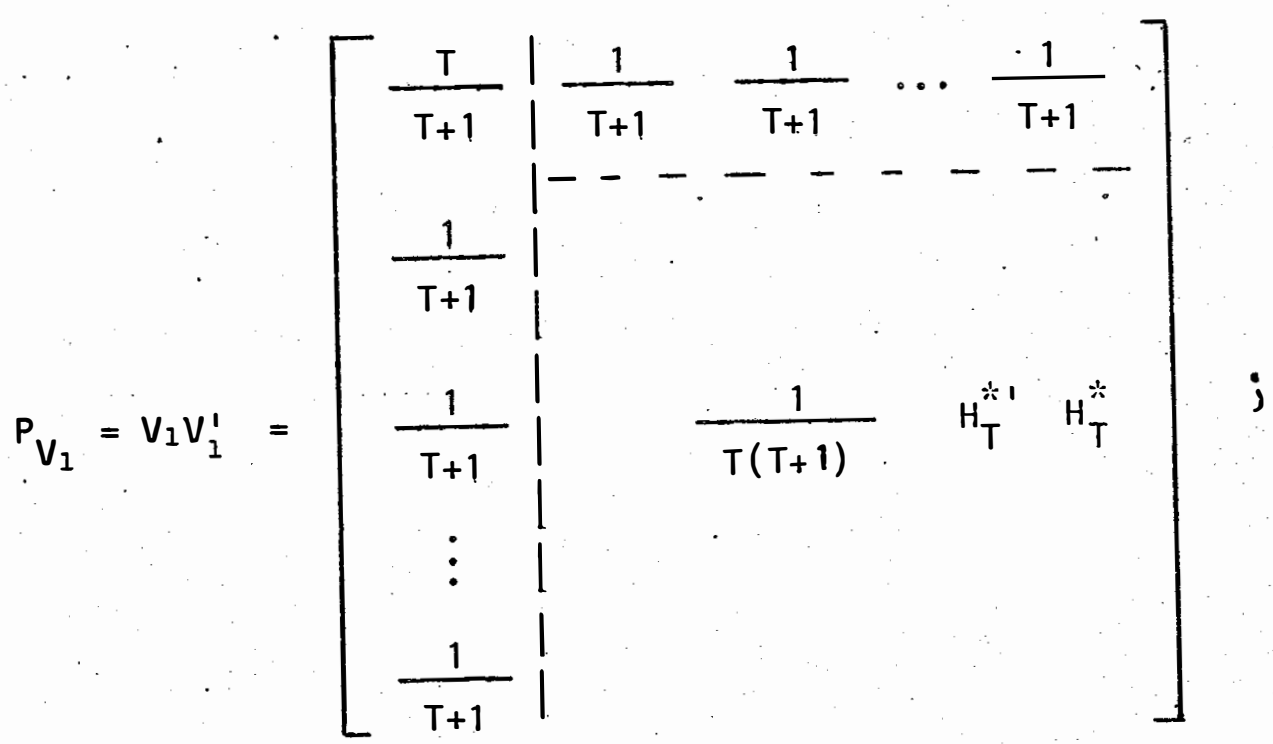

pondo-se $\frac{1}{T+1}$ em evidência e das propriedades das matrizes de Helmert, vem: 


$$
P_{V_{1}}=\frac{1}{T+1}\left[\begin{array}{cccccc}
T & 1 & 1 & 1 & \ldots & 1 \\
1 & T & -1 & -1 & \ldots & -1 \\
1 & -1 & T & -1 & \ldots & -1 \\
1 & -1 & -1 & T & \ldots & -1 \\
\vdots & \vdots & \vdots & \vdots & & \vdots \\
1 & -1 & -1 & -1 & & T
\end{array}\right]
$$

$$
\text { 4.6.3.3. A MATRIZ V } S_{1}^{-1} U_{1}^{\prime}
$$

Ee acordo com o resultado obtido em [4.39], tem-se que:

$S_{1}^{-1}=\left[\begin{array}{ccccc}\frac{1}{\sqrt{(T+1) R}} & \phi & \phi & \cdots & \phi \\ \phi & \frac{1}{\sqrt{R}} & \phi & \cdots & \phi \\ \phi & \phi & \frac{1}{\sqrt{R}} & \cdots & \phi \\ \vdots & \vdots & \vdots & & \vdots \\ \phi & \phi & \phi & \cdots & \frac{1}{\sqrt{R}}\end{array}\right]$


Logo, de [4.41],

$$
V_{1} S_{1}^{-1}=\frac{1}{\sqrt{R}}\left[\begin{array}{ccccc}
\frac{T}{(T+1) T} & \phi & \phi & \cdots & \phi \\
\frac{1}{(T+1) \sqrt{T}} & \frac{1}{\sqrt{2}} \frac{1}{\sqrt{6}} & \cdots & \frac{1}{\sqrt{T(T-1)}} \\
\frac{1}{(T+1) \sqrt{T}} & \frac{-1}{\sqrt{2}} & \frac{1}{\sqrt{6}} & \cdots & \frac{1}{\sqrt{T(T-1)}} \\
\frac{1}{(T+1) \sqrt{T}} & \phi & \frac{-2}{\sqrt{6}} & \cdots & \frac{1}{\sqrt{T(T-1)}} \\
\vdots & \vdots & \vdots & & \vdots \\
\frac{1}{(T+1) \sqrt{T}} & \phi & \phi & \cdots & \frac{-(T-1)}{\sqrt{T(T-1)}}
\end{array}\right]
$$

ou seja,

$$
V_{1} S_{1}^{-1}=\frac{1}{\sqrt{R}}\left[\begin{array}{cccc}
\frac{T}{(T+1)} \sqrt{T} & 1 & \\
& & 1 & \\
\frac{T}{(T+1)} \sqrt{T} & 1 & H_{T}^{* 1} \\
\vdots & 1 & 1 \\
\frac{1}{(T+1)} & 1
\end{array}\right] \quad \text { [4.47] }
$$


de acordo com [4.40]

$V_{1} S_{1}^{1} U_{1}^{\prime}=\frac{1}{\sqrt{R}}\left[\begin{array}{ccc}\frac{T}{(T+1) \sqrt{T}} & \vdots & \phi \\ \frac{1}{(T+1) \sqrt{T}} & \vdots & H_{T}^{* 1} \\ \vdots & \vdots & \vdots \\ 1 & \vdots \\ \frac{1}{(T+1) \sqrt{T}} & \vdots\end{array}\right] \frac{1}{\sqrt{R}}\left(\left[T_{1}^{h_{1}} H_{T}^{* 1}\right]: \otimes E_{R} E_{R}\right.$

efetuando-se o-produto matricial

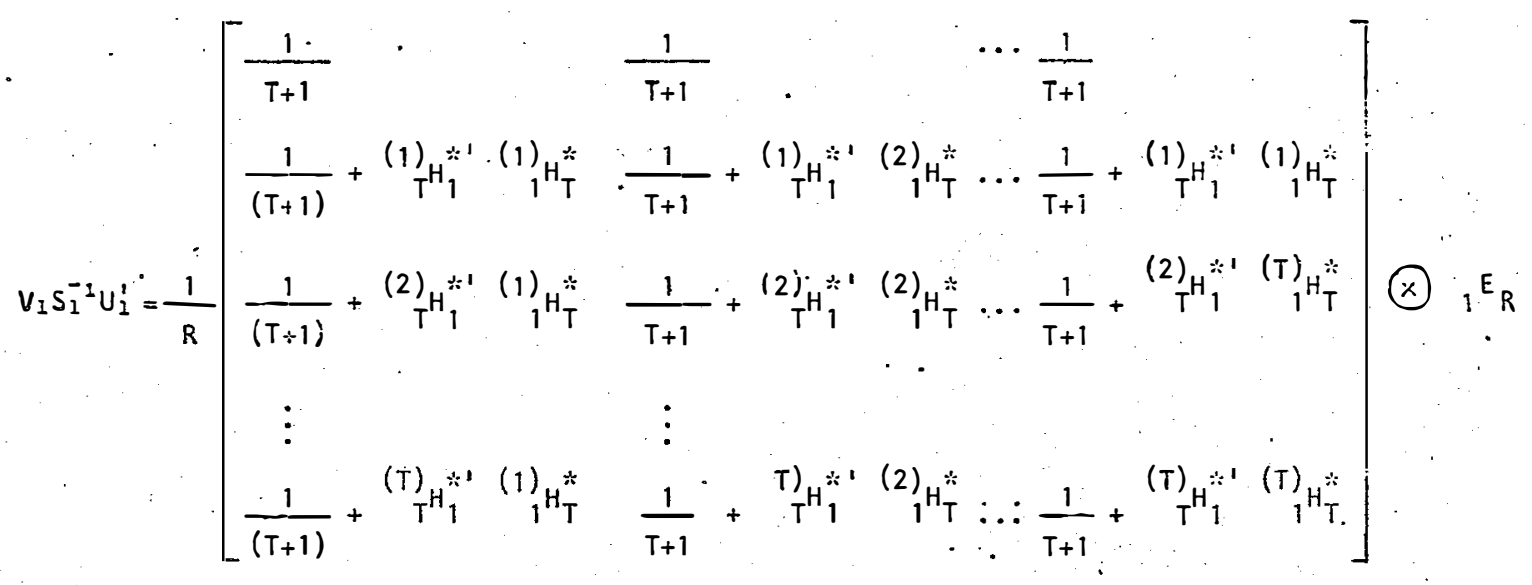

de acordo com [4.35],

$$
\text { (i) }{ }_{T} H_{1}^{*}(j){ }_{1}^{*} H_{T}^{*}=\left\{\begin{array}{ccc}
-\frac{T-1}{T} & \text { se } & i=j \\
\frac{-1}{T} & \text { se } & i \neq j
\end{array}\right.
$$


tendo-se então que:

$$
V_{1} S_{1}^{-1} U_{1}^{\prime}=\frac{1}{R}\left[\begin{array}{cccc}
\frac{1}{T+1} & \frac{1}{T+1} & \cdots & \frac{1}{T+1} \\
\frac{T}{T+1} & \frac{-1}{T+1} & \cdots & \frac{-1}{T+1} \\
\frac{-1}{T+1} & \frac{T}{T+1} & \cdots & \frac{-1}{T+1} \\
\vdots & \vdots & \ddots & \vdots \\
\frac{-1}{T+1} & \frac{-1}{T+1} & \cdots & \frac{T}{T+1}
\end{array}\right]_{P} \otimes E_{R}
$$

$$
V_{1} S_{1}^{-1} U_{1}^{\prime}=\frac{1}{R} \frac{1}{P}\left[\begin{array}{rrrrr}
1 & 1 & 1 & \ldots & 1 \\
T & -1 & -1 & \ldots & -1 \\
-1 & T & -1 & \ldots & -1 \\
-1 & -1 & T & \ldots & -1 \\
\vdots & \vdots & \vdots & & \vdots \\
-1 & -1 & -1 & & T
\end{array}\right]_{P} \otimes V_{1} E_{R}
$$

onde $p=T+1$

$$
\begin{aligned}
& \text { 4.6.3.4. A MATRIZ } V_{1} S_{1}^{-2} V_{1}^{1} \\
& \text { Pós-multiplicando }[4.47] \text { por } s_{1}^{-1} \text {, tem-se: }
\end{aligned}
$$




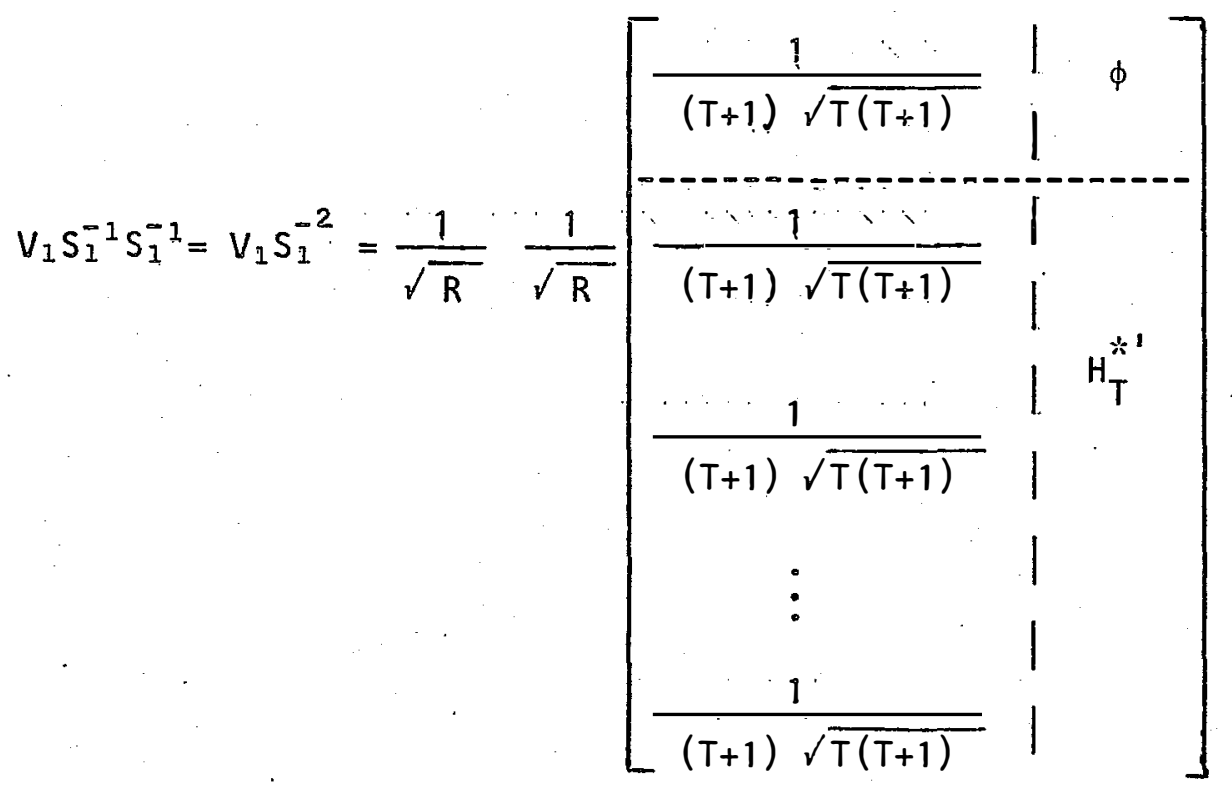

pós-multiplicando [4.49] por $V_{1}^{\prime}$, tem-se:

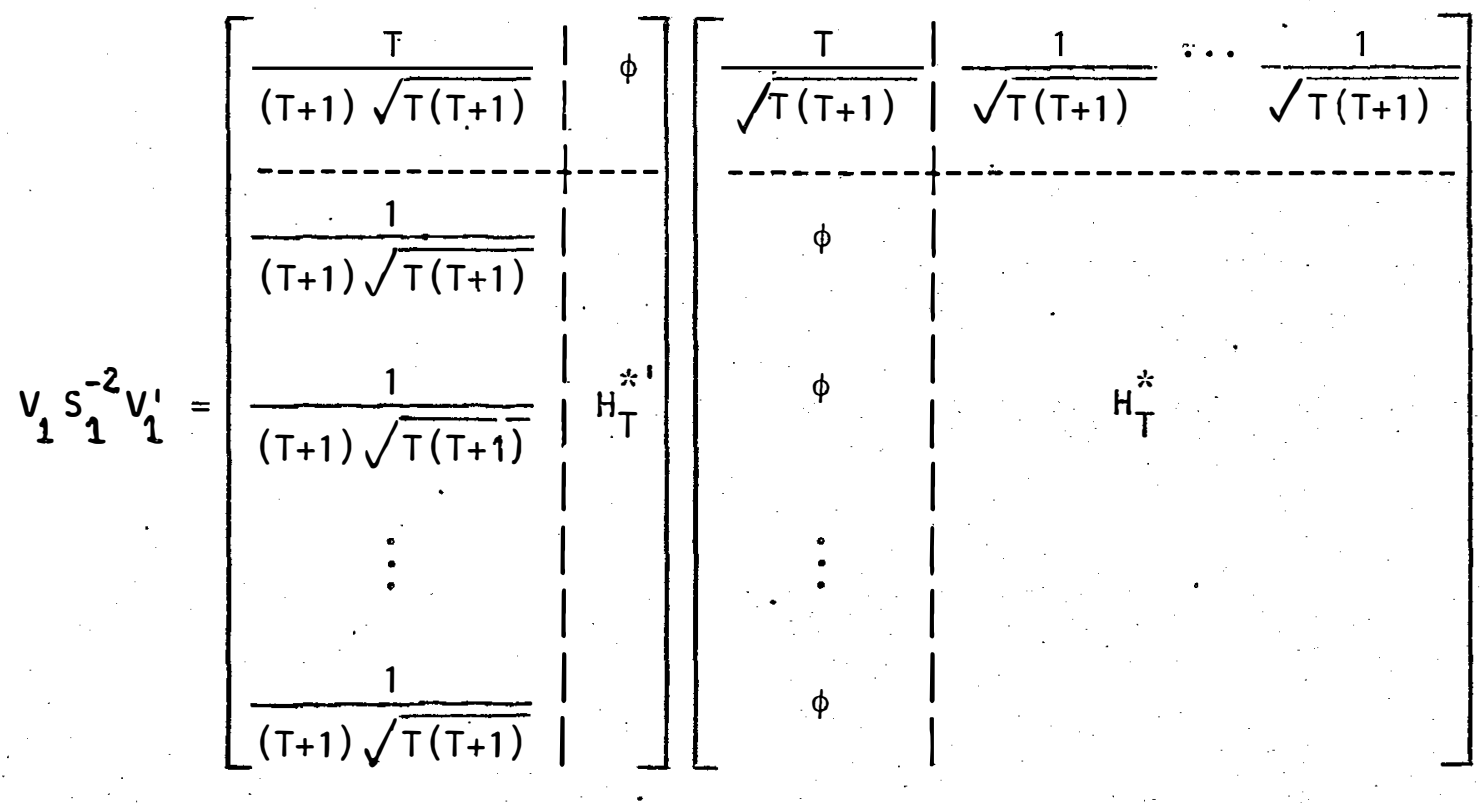

efetuando-se o produto, 


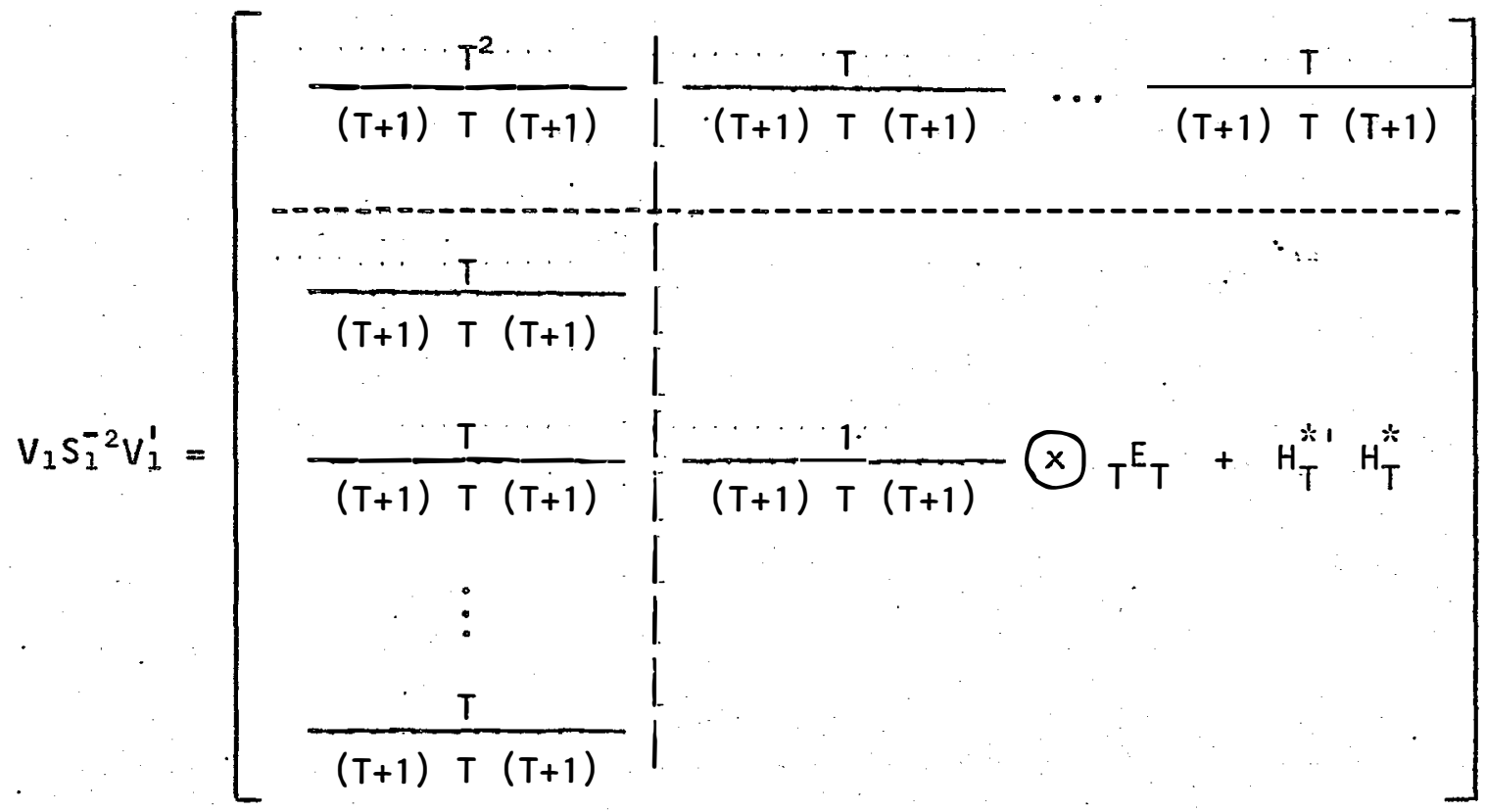

sendo

$$
\begin{aligned}
& \frac{1}{(T+1) T(T+1)}+\frac{T-1}{T}=\frac{1+(T-1)(T+1)^{2}}{T(T+1)^{2}} \\
& \frac{1 \quad \cdot}{(T+1) T(T+1)}-\frac{1}{T}=\frac{1-(T+1)^{2}}{T(T+1)^{2}} \quad ; \text { tem-se então, }
\end{aligned}
$$

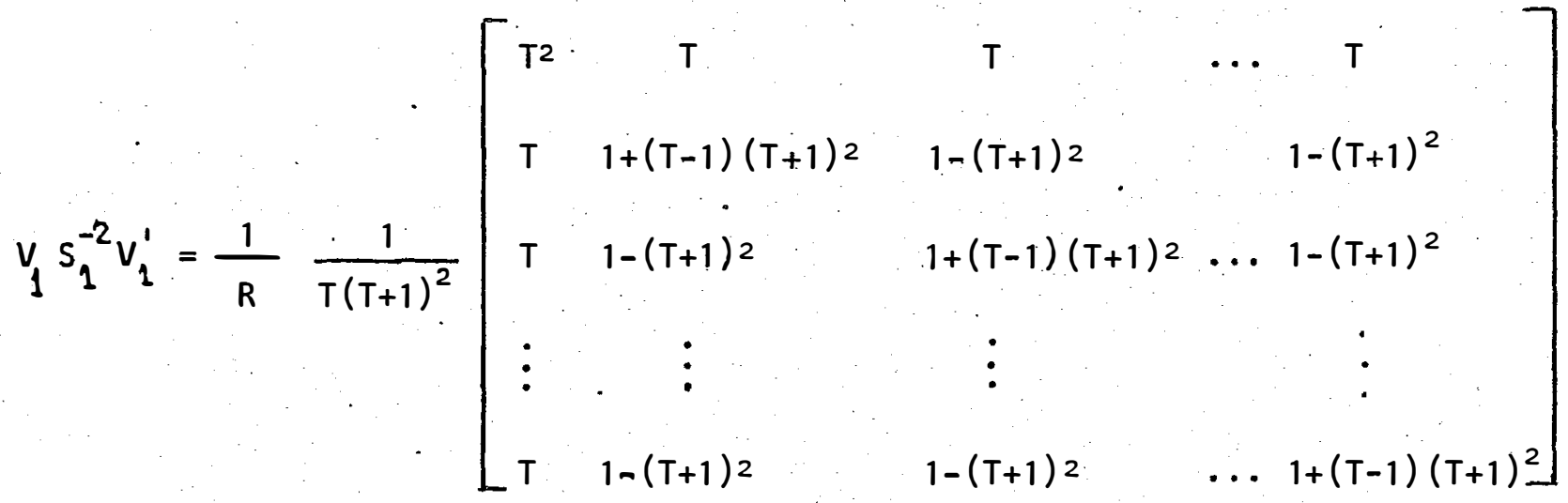

de ${ }_{n} X_{p}$, tem-se: $T+1=p ; R \cdot T=n ;$ logo: 
.86.

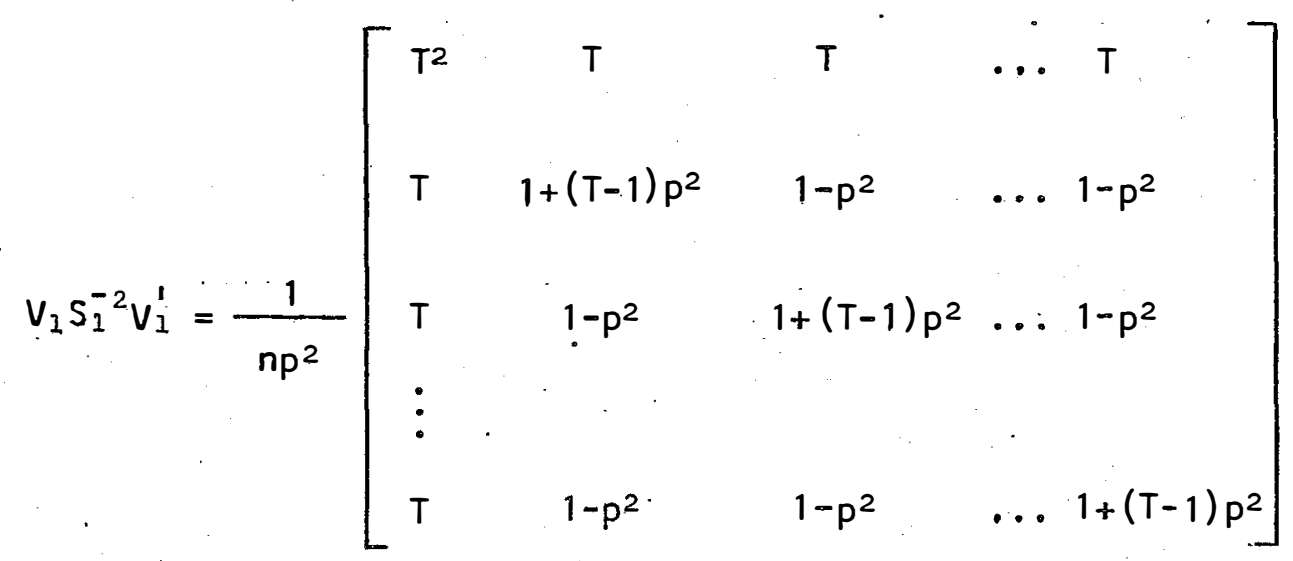

[4.50]

4.6.1: DESCRICÃO DO MODELO

Seja o modelo de Gauss-Markov, de acordo com $[4.1]$.

$$
y=x \theta+e
$$

Seja também a caracterização

$$
y_{i j}=\mu+t_{i}+e_{i j}
$$

$$
\begin{aligned}
& \text { onde, para } i=1,2, \ldots, T \text { tratamentos } \\
& \text { e } \quad \mathrm{j}=1,2, \ldots, \mathrm{R} \text { rejpetições }
\end{aligned}
$$

tem-se que:

$y_{i j}$ é o valor observado na j-ésima: repetição do i-ésimo tra tamento. 
$\mu \quad$ é uma constante inerente a todas as observações.

$t_{i} \quad \dot{e}$ o efeito do i-ésimo tratamento.

$e_{i j}$ é o erro aleatório atribuído à observação $y_{i j}$, tal que $e_{i j} \sim N\left(0,1 \sigma^{2}\right)$

desse modo, tem-se a seguinte configuração:

$n \dot{x}_{P}=\left[\begin{array}{ccccc}1 & 1 & 0 & \ldots & 0 \\ \ldots & \ldots & \ldots & \ldots & \ldots \\ 1 & 1 & 0 & \ldots & 0 \\ 1 & 0 & 1 & \ldots & 0 \\ \ldots & \ldots & \ldots & \ldots & \ldots \\ 1 & 0 & 1 & \ldots & 0 \\ \ldots & \ldots & \ldots & \ldots & \ldots \\ \ldots & \ldots & \ldots & \ldots & \ldots \\ 1 & 0 & 0 & \ldots & 1 \\ \ldots & \ldots & \ldots & \ldots & \ldots \\ 1 & 0 & 0 & \ldots & 1\end{array}\right] ; \quad y=\left[\begin{array}{c}y_{11} \\ \ldots \\ y_{1 R} \\ y_{21} \\ \ldots \\ y_{2 R} \\ \ldots \\ \ldots \\ y_{T_{1}} \\ \ldots \\ y_{T R}\end{array}\right] ; \theta=\left[\begin{array}{c}\mu \\ t_{1} \\ t_{2} \\ \ldots \\ t_{T}\end{array}\right] ; e=\left[\begin{array}{c}e_{11} \\ \ldots \\ e_{1 R} \\ e_{21} \\ \ldots \\ e_{2 R} \\ \cdots \\ \ldots \\ e_{T 1} \\ \ldots \\ e_{T R}\end{array}\right]$

No delineamentó em questão, a solução, de acordo com [4.7], dada por

$$
\theta^{0}=V_{1} S_{1}^{-1} U_{1}^{1} \quad y
$$

do sistema de equações [4.5] será, de acordo com [4.48], dada por: 


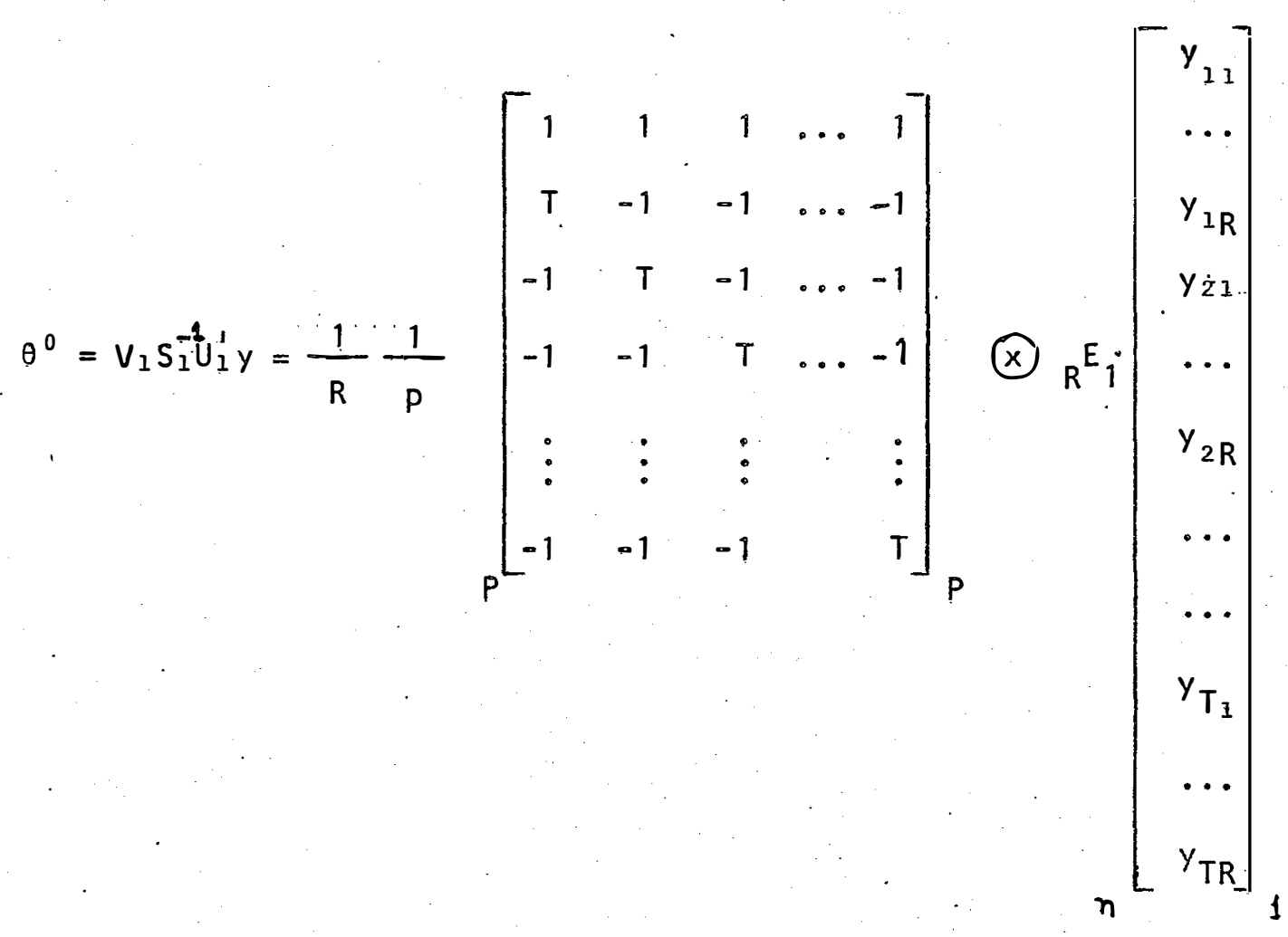

resultando

$$
\theta^{0}=\left[\begin{array}{cc}
\bar{y}_{\cdots} & \\
\bar{y}_{1} & -\bar{y}_{n} \\
\bar{y}_{2} & -\bar{y}_{\cdots} \\
\cdots \\
\bar{y}_{T}-\bar{y}_{1}
\end{array}\right]=\left[\begin{array}{c}
\mu^{0} \\
t_{1}^{0} \\
t_{2}^{0} \\
\cdots \\
t_{T}^{0}
\end{array}\right]
$$

e de acordo com [4.8], a aproximação de mínimos quadrados pa ra $\hat{y}$ é dada por: 


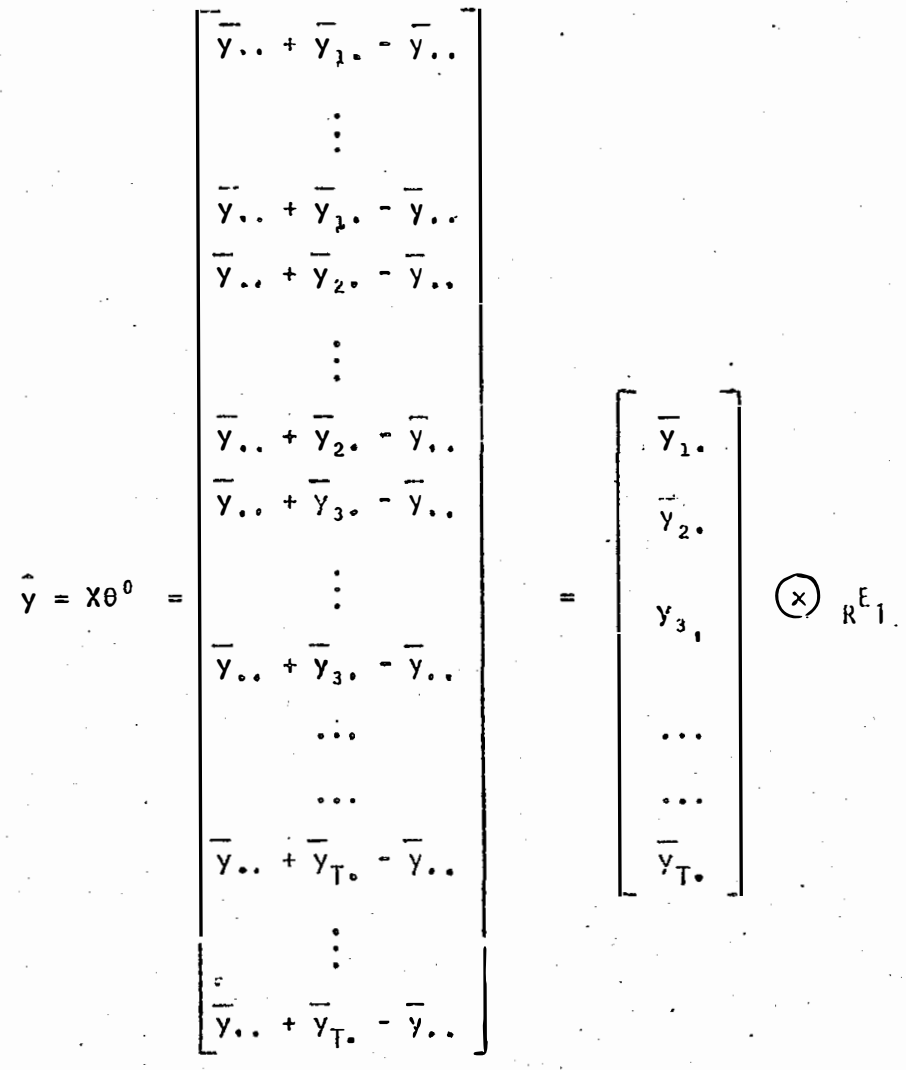

\subsubsection{EST IMAB ILIDADE}

De acordo com a proposição 4 tem-se que as funções dos parâmetros $\lambda^{\prime} \theta$, tal que $\lambda^{\prime} V_{1} V_{1}^{\prime}=\lambda^{\prime}=\lambda^{\prime} P_{v_{1}}$ são. es timáveis.

$$
\begin{gathered}
\text { Do resultado [4.46], tem-se que: } \\
\lambda^{\prime} V_{1} V_{1}^{\prime}=\lambda^{\prime} P_{V_{1}}=\lambda^{\prime} \frac{1}{T+1}\left[\begin{array}{rrrrr}
T & 1 & 1 & \ldots & 1 \\
1 & T & -1 & \ldots & -1 \\
1 & -1 & T & \ldots & -1 \\
\vdots & \vdots & \vdots & & \vdots \\
1 & -1 & -1 & \ldots & -1
\end{array}\right]
\end{gathered}
$$

Proposição 7. No modelo do presente estudo, uma condição necessäria e suficiente para que

$$
P_{V_{I}} \lambda=\lambda\left(\text { ou } \lambda^{\prime} P_{V_{I}}=\lambda^{\prime}\right) \text { é que }
$$




$$
\sum_{i=1}^{p} \lambda_{i}=2 \lambda_{1}
$$

Prova:

$$
\text { Dado } \lambda=\left[\begin{array}{c}
\lambda_{1} \\
\lambda_{2} \\
\vdots \\
\lambda_{P}
\end{array}\right] ; \text { tem-se então que a }
$$

seguinte igualdade matricial se verifica, ou seja, a função $\lambda^{\prime} \theta$ e é estimävel.

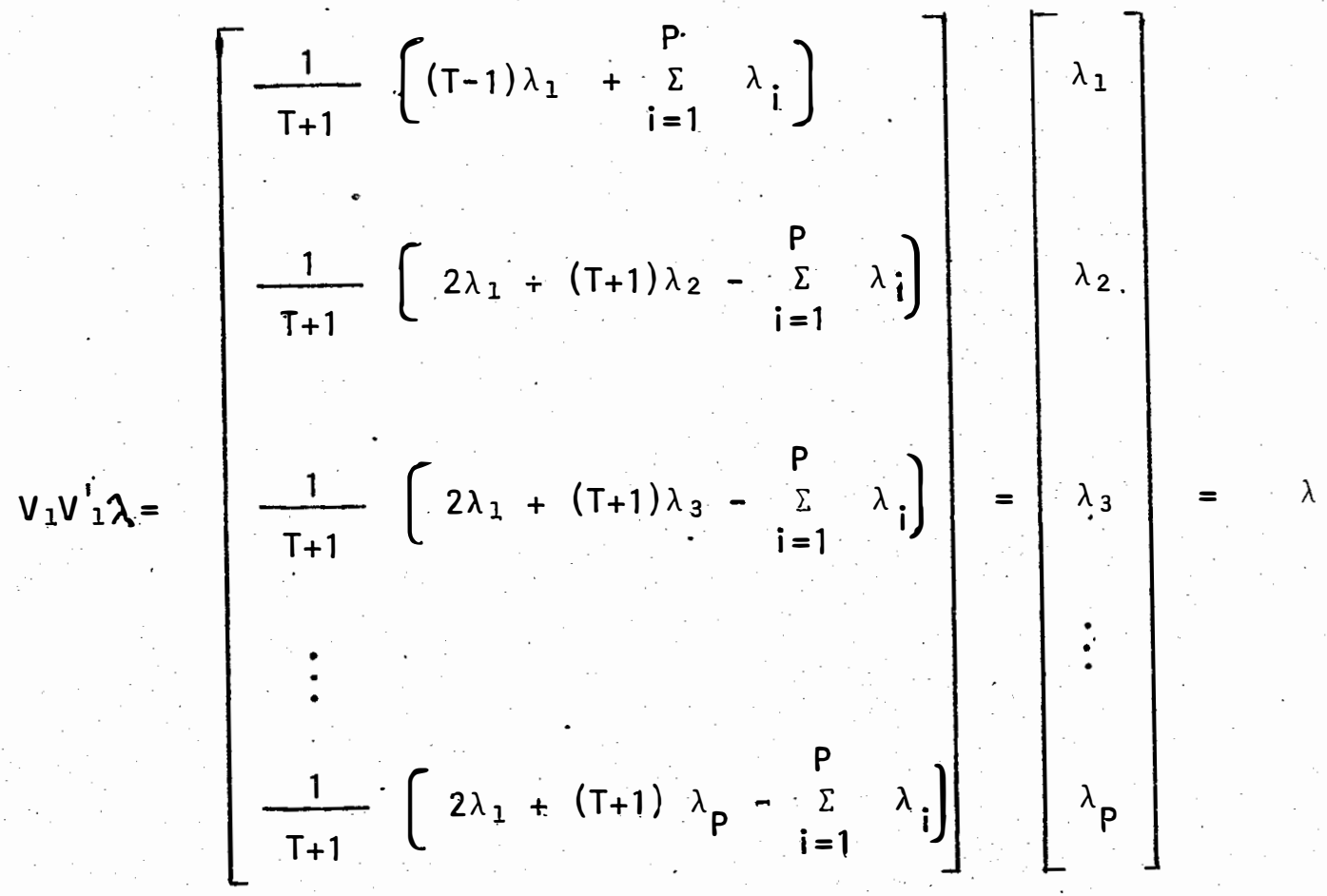

se e somente se, 
a) $\frac{1}{T+1}\left[(T-1) \lambda_{1}+\sum_{i=1}^{P} \lambda_{i}\right]=\lambda_{I}$
b) $\frac{1}{T+1}\left[2 \lambda_{1}+(T+1) \lambda_{j}-\sum_{i=1}^{P} \lambda_{i}\right]=\lambda_{j} ; j=2,3, \ldots, p$
Assim, a) e b) serão verdadeiros se e só se
$\quad \begin{gathered}P \\ i=1\end{gathered} \lambda_{i}=2 \lambda_{I}$

completando a demonstração.

Corolärio 9. Se $\lambda_{1}=\phi, V_{1} V_{1}^{\prime} \lambda=\lambda$ se e sö se $\lambda^{\prime} \theta^{0}$ for um contraste, i.é.,

$$
\sum_{i=1}^{p} \lambda_{i}=\phi .
$$

$$
\text { 4.6.3. NO MODELO } y=\left[\begin{array}{l:l}
x_{1} & x_{2}
\end{array}\right]\left[\begin{array}{c}
\theta_{1} \\
\cdots \\
\theta_{2}
\end{array}\right]+e
$$

Nesse modelo, a matriz de planejamento tem a seguinte configuração 


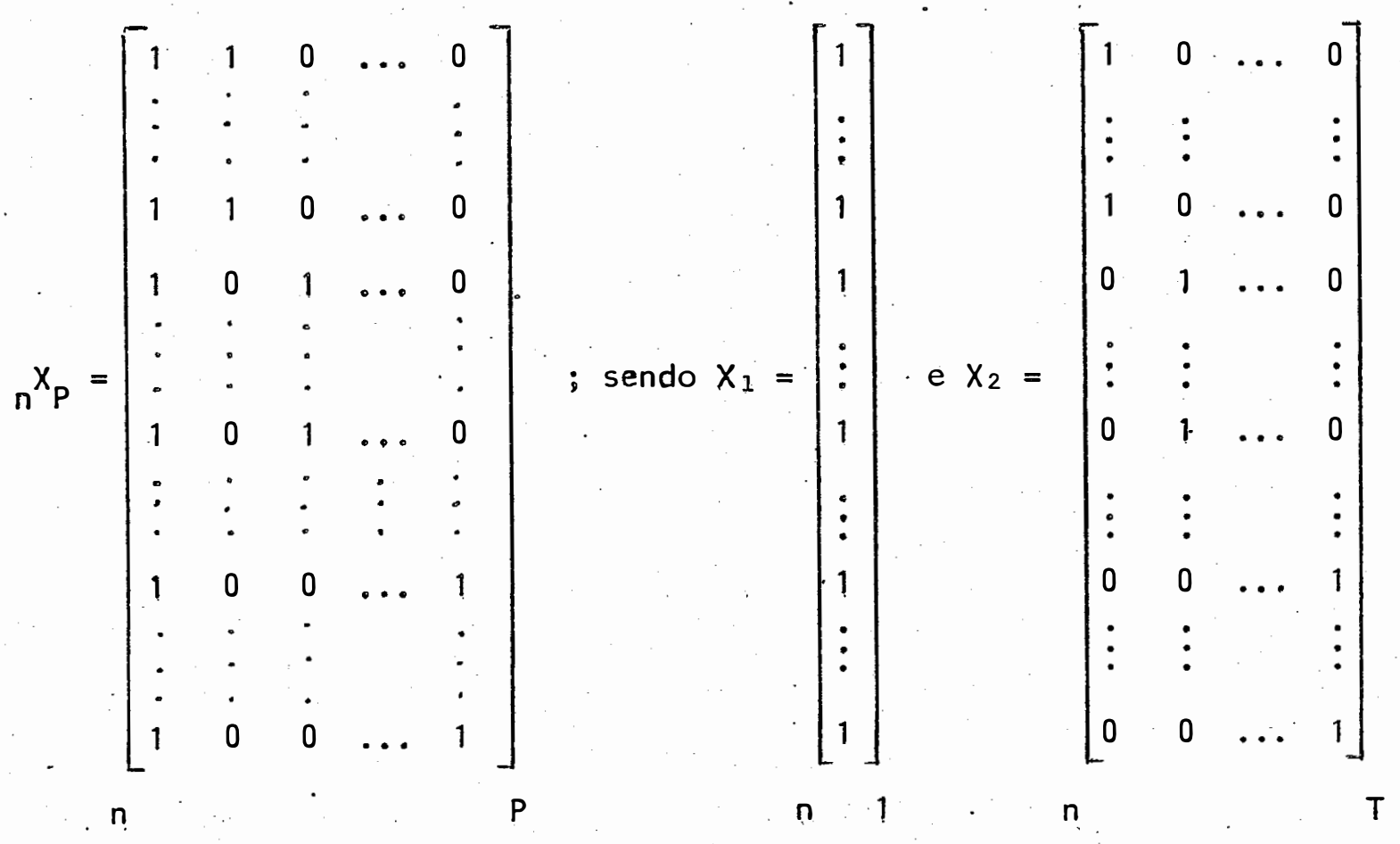

4.6.3.1. A D.V.S. DA MATRIZ $X_{2}=U_{12} S_{12} V_{12}^{\prime}$

4.6.3.1.2. Os autovalores não nulos $\sin _{121}^{2}, \sin _{122}^{2}, \ldots$ $s_{12 T}^{2}$ de $X_{2}^{\prime} x_{2}\left(x_{2} X_{2}^{\prime}\right.$, respectivamente $)$

Tem-se que:

$$
X_{2}^{1} X_{2}=\left[\begin{array}{ccccc}
R & 0 & 0 & \cdots & 0 \\
0 & R & 0 & \cdots & 0 \\
0 & 0 & R & \cdots & 0 \\
\vdots & \vdots & \vdots & & \vdots \\
0 & 0 & 0 & \cdots & R
\end{array}\right] ;
$$


verifica-se imediatamente que os autovalores de $x_{2}^{1} x_{2}$, são:

$$
\begin{aligned}
& s_{121}^{2}=R \\
& s_{122}^{2}=R \\
& \vdots \\
& s_{1: 2 T}^{2}=R
\end{aligned}
$$

portanto,

$$
S_{12}=\left[\begin{array}{cccc}
\sqrt{R} & \phi & \ldots & \phi \\
\phi & \sqrt{R} & \ldots & \phi \\
\vdots & \vdots & & \vdots \\
\phi & \phi & \ldots & \sqrt{R}
\end{array}\right]=\sqrt{R} \quad(T) ;
$$

4.6.3.1.2. Os autovetores normalizados de $x_{2}^{1} x_{2}$, correspondentes aos autovalores não nulos

$$
s_{121}^{2}, s_{122}^{2}, \cdots, s_{12 T}^{2}
$$

A matriz $v_{21}$ dos autovetores $v_{121}, v_{122}, \ldots$, $v_{12 T}$; relativos aos autovalores positivos $s_{121}^{2}, s_{122}^{2}, \ldots$, $\mathrm{s}_{12 \mathrm{~T}}^{2}$ de $\mathrm{X}_{2}^{1} \mathrm{X}_{2}$, de acordo com a teoria vista no desenvolvimento do algorittmo ASSIZ CARVALHO/IEMMA, será dadá por: 


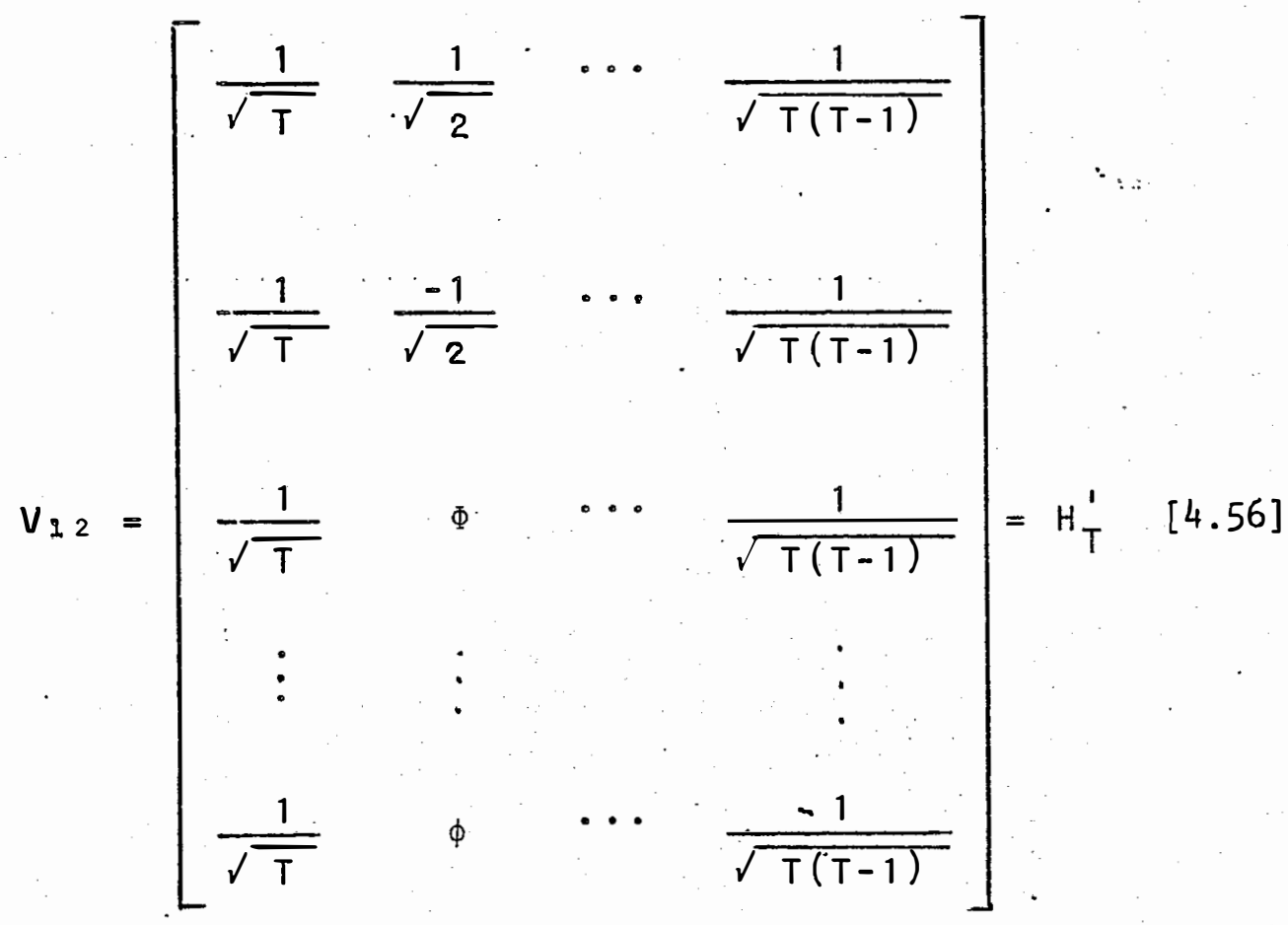

4.6.3.1.3. Os autovetores normalizados de $x_{2} x_{2}^{\prime}$, correspondentes aos autovalores não nulos

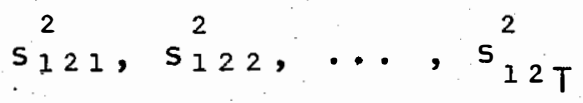

A matriz Ul2 dos autovetores normalizados $u_{121}, u_{122}, \ldots, u_{12 T} ;$ relativos aos autovalores positivos $s_{121}^{2}, s_{122}^{2}, \ldots, s_{12 T}^{2}$ de $x_{2} X_{2}^{1}$, serä dada por:

$$
u_{i}=\frac{1}{s_{i}} x_{2} \quad v_{i} \quad e
$$

pelo algoritmo ASSIZ CARVALHO/IEMMA, 


$$
\begin{aligned}
& n_{T}=\frac{1}{\sqrt{R}}\left(H_{T}^{\prime} \otimes R_{R} E_{1}\right) \\
& 4.6 .3 .1 .4 . \text { Verificando } U_{12} S_{12} V_{12}^{1}=X_{2} \\
& \text { De [4.55], [4.56] e [4.57] } \\
& S_{12}=\sqrt{R} 1(T) \\
& V_{12}=H_{T}^{1} \\
& U_{12}=\frac{1}{\sqrt{R}}\left(H_{T}^{\prime} \otimes R_{R} E_{1}\right.
\end{aligned}
$$

- portanto,

$$
\begin{aligned}
U_{12} S_{12} V_{12}^{\prime} & =\frac{1}{\sqrt{R}}\left(H_{T}^{\prime} \otimes E_{R}\right)_{1} \sqrt{R} I_{(T)} H_{(T)} \\
& =\frac{\sqrt{R}}{\sqrt{R}}\left(H_{T}^{\prime} \otimes{ }_{R} E_{1}\right) H_{T} \\
& =\left(H_{T}^{\prime} \cdot H_{T}\right) \otimes R_{R} E_{1} \\
& =I_{(T)} \otimes{ }_{R} E_{1} \\
& =X_{2}
\end{aligned}
$$

concluindo à verificação. 


$$
\text { 4.6.3.2: A D.V.S. DA MATRIZ } X_{1}=U_{11} S_{11} V_{11}^{\prime}
$$

De maneira anāloga ao 4.3 .3 .1 , tem-se que a matriz $S_{11}$, correspondente a raiz quadrada dos autovalores positivos de $X_{1} X_{1}^{\prime}$, é dada por,

$$
S_{11}=\left[s_{111}^{2}\right]=[\sqrt{T R}]_{1} ;
$$

a matriz $U_{11}$ dos autovetores de $X_{1} X_{1}^{\prime}$ correspondente ao autovetor não nulo $s_{111}^{2}$, é dado por

$$
U_{11}=\frac{1}{\sqrt{T R}} \otimes{ }_{n} E_{1} \quad ;
$$

a matriz $V_{11}$ dos autovetores de $X_{1}^{1} X_{1}$ correspondente ao autovetor não nulo $s_{111}^{2}$, é dado por

$$
v_{11}=[1]
$$

e então,

$$
\begin{aligned}
U_{11} S_{11} \cdot V_{11}^{\prime} & =\left[\frac{1}{\sqrt{T R}} \otimes{ }_{n} E_{1}\right] \sqrt{T R} \cdot 1 \\
& =\left[\begin{array}{c}
1 \\
1 \\
\vdots \\
1
\end{array}\right]=x_{1}
\end{aligned}
$$


4.6.3.3. PROJETORES ORTOGONAIS

4.6.3.3.1. O projetor ortogonal em $C\left(X_{1}\right): P_{U_{11}}$

$$
P_{U_{11}}=x_{1} x_{1}^{+}=U_{11} U_{11}^{\prime}
$$

De acordo com [4.58],

$$
P_{U_{11}}=\left[\frac{1}{\sqrt{T R}} \otimes{ }_{n} E_{1}\right]\left[\frac{1}{\sqrt{T R}} \otimes{ }_{1} E_{n}\right]
$$$$
\therefore \quad P_{U_{11}}=\frac{1}{T R} n^{E}{ }_{n}
$$

$$
\begin{aligned}
& \text { 4.6.3.3.2: } 0 \text { projetor ortogonai em } C\left(X_{2}\right): P_{U_{12}} \\
& P_{U_{12}}=X_{2} X_{2}^{+}=U_{12} U_{12}^{\prime} \\
& \text { De acordo com [4.57] } \\
& P_{U_{12}}=\left(\frac{1}{\sqrt{R}} H_{T}^{\prime} \otimes{ }_{R} E_{1}\right)\left(\frac{1}{\sqrt{R}} H_{T}^{\prime} \otimes R^{E_{1}}\right]^{\prime} \\
& =\frac{1}{R} H_{T}^{\prime} H_{T} \otimes{ }_{R}{ }_{R} \\
& \therefore P_{U_{12}}=\frac{1}{R}{ }^{1}(T) \otimes R_{R}{ }^{2} \text {. }
\end{aligned}
$$




$$
\begin{aligned}
& \text { 4.6.3.4. FORMAS QUADERATTICAS } \\
& 4 \cdot 6 \cdot 3 \cdot 4 \cdot 1 \text {. A forma quadrätica } y^{\prime} P_{U_{11}} y
\end{aligned}
$$

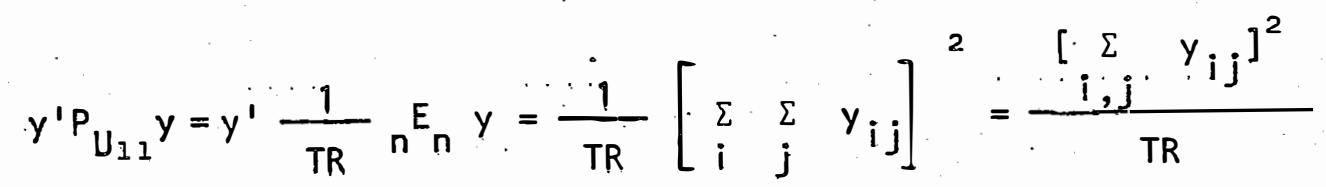

$$
\begin{aligned}
& \therefore \quad y^{\prime} P_{U_{11}} y=\frac{\left[\sum_{i, j} y_{i j}\right]^{2}}{T R}=\frac{1}{T R} y^{2} . \\
& \text { 4.6.3.4.2. A forma quadrätica } y^{\prime}\left(P_{U_{1}}-P_{U_{11}}\right) y \\
& y^{\prime}\left(P_{U_{1}}-P_{U_{11}}\right) y=y^{\prime} P_{U_{1}} y-y^{\prime} P_{U_{11}} y \\
& =\frac{1}{R} \sum_{i=1}^{T} y_{i}^{2} \cdot-\frac{1}{T R} y^{2} . \\
& \text { 4.6.3.4.3. A forma quadrätica } y^{\prime}\left(1-P_{U_{1}}\right) y \\
& y^{\prime}\left(1-P_{U_{1}}\right) y=y^{\prime} y-y^{\prime} P_{U_{Z}} y \\
& =\sum_{i . j} y_{i j}^{2}-\frac{1}{R} \sum_{i=1}^{T} y_{i}^{2}
\end{aligned}
$$


4.6.4. DISTRIBUIÇÃO DAS FORMAS QUADRÁTICAS

$$
\begin{aligned}
& \text { De acordo com SEARLE (1971), } \\
& W=\frac{\frac{x_{m}^{2}}{m}}{\frac{x_{n-T}^{2}}{n-T}} \sim{ }_{m, n-T}^{F}
\end{aligned}
$$

Do resultado [4.22], tèm-se que

$$
\begin{aligned}
& y^{\prime}\left(P_{U_{1}}-P_{U_{11}}\right) y \\
& \frac{\sigma^{2}}{\sigma^{2}} \cdot\left[T-\operatorname{tr}\left(P_{U_{11}}\right) ; \frac{1}{2 \sigma^{2}}\left(\theta^{\lrcorner} V_{1} S_{1}^{2} V_{1}^{\prime} \theta-\theta \theta^{\prime} V_{1} S_{1} U_{1}^{\prime} P_{U_{11}} U_{1} S_{1} V_{1}^{\prime} \theta\right)\right] \\
& \text { resultado [4.60] segue-se que : } \\
& \theta^{\prime} V_{1} S_{1} U_{1}^{\prime} P_{U_{11}} U_{1} S_{1} V_{j} \theta=\theta^{\prime} X^{\prime} \frac{1}{T R} \cdot E_{n} \times \theta \\
& =\theta^{\prime} X^{\prime}{ }_{n} E_{n} X \theta \frac{1}{T R} \\
& =\theta^{\prime} T R X^{\prime} X \theta \frac{1}{T R} \\
& =\theta \cdot x \cdot x \theta
\end{aligned}
$$$$
\text { Como } \quad \theta^{\prime} V_{1} S_{1} U_{1} P_{U_{11}} u_{1} s_{1} V_{1} \theta=\theta^{\prime} \times P_{U_{11}} \times \theta \text {, do }
$$ 
entäo, conclui-se que:

$$
\frac{y^{\prime}\left(P_{U_{1}}-P_{U_{11}}\right) y}{\sigma^{2}} \sim x^{2}\left[T-\operatorname{tr}\left(P_{U_{11}}\right)\right]
$$

e de acordo com o resultado [4.21]

$$
\frac{y^{\prime}\left(1-P_{U_{1}}\right) y}{\sigma^{2}} \sim x_{[n-T]}^{2}
$$

então, tem-se:

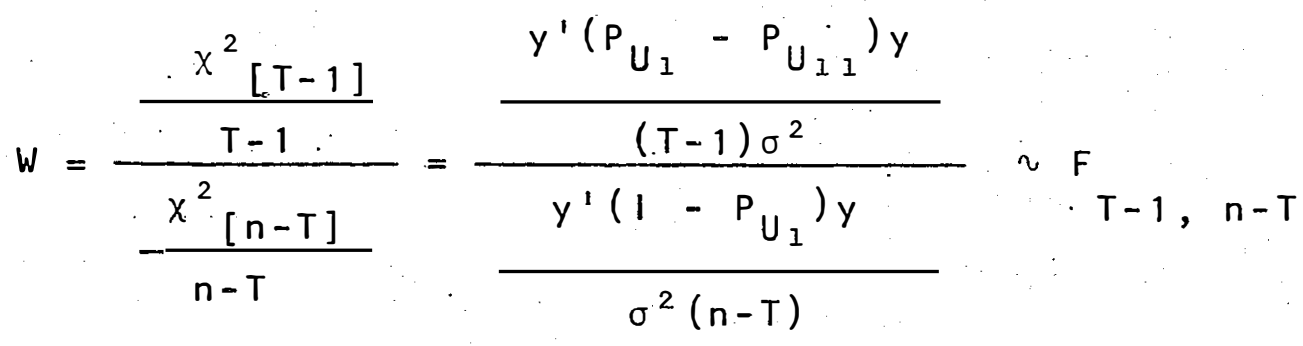

ou seja

$$
\frac{\frac{y^{\prime}\left(P_{U_{1}}-P_{U_{I I}}\right) y}{T-1}}{\frac{y^{\prime}\left(1-P_{U_{I}}\right) y}{n-T}} \sim F F-T-1, n-T
$$


Dos resultados obtidos, tem-se o seguinte qua dro para a Anälise de Variância.

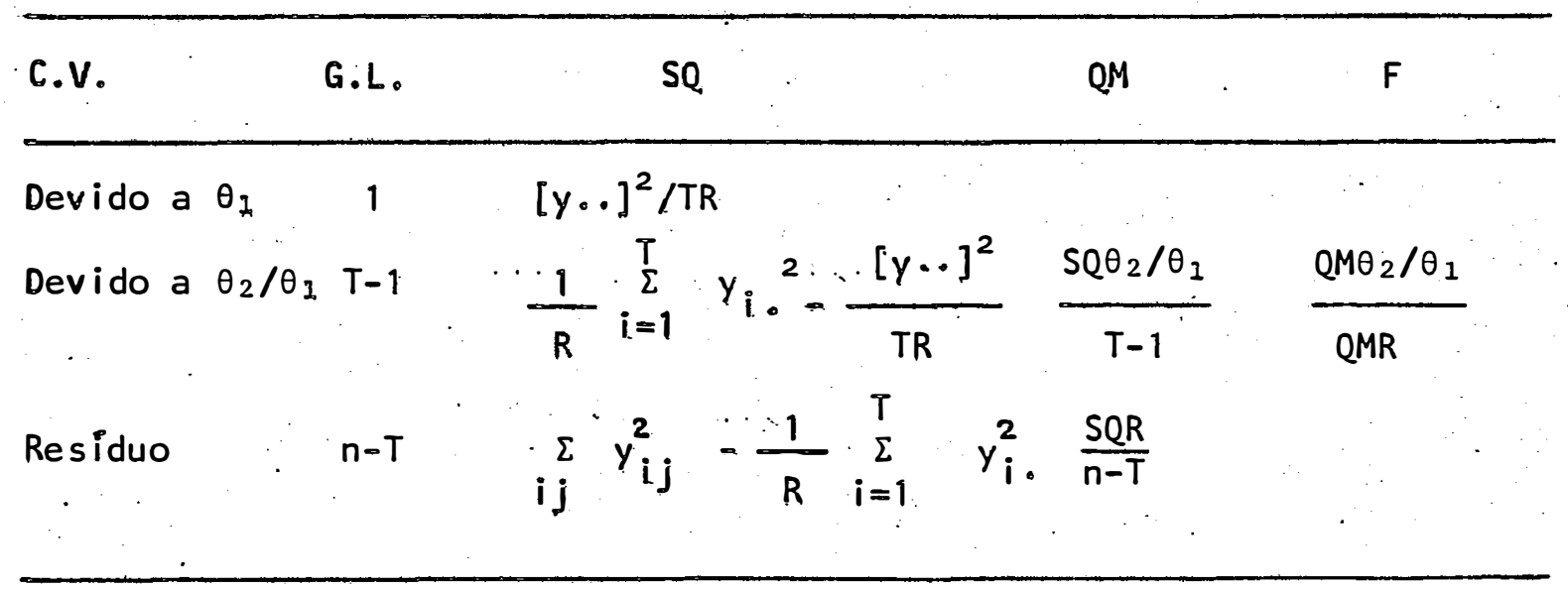

Tota'i

$n$

o qual è adequado para inferências sobre tratamentos $\left(\theta_{2} / \theta_{2}\right)$

\section{6 .5 . ESTIIMACÃO POR INTERVALO E POR REGIÃO}

De acordo com $[4.24]$, uma estimativa por intervalo ao nível de confiança $1-\alpha$, para $\lambda^{\prime} \theta$ estimável, pode ser obtida de:

$$
\operatorname{lC}\left[\lambda^{\prime} \theta\right]_{1-\alpha}=\widehat{\lambda^{\prime} \theta} \pm t t_{[\alpha / 2 ; N-T]} \sqrt{\hat{V}\left(\lambda^{\prime} \theta^{0}\right)} ; \text { onde [4.64] }
$$

de acordo com [4.50], 


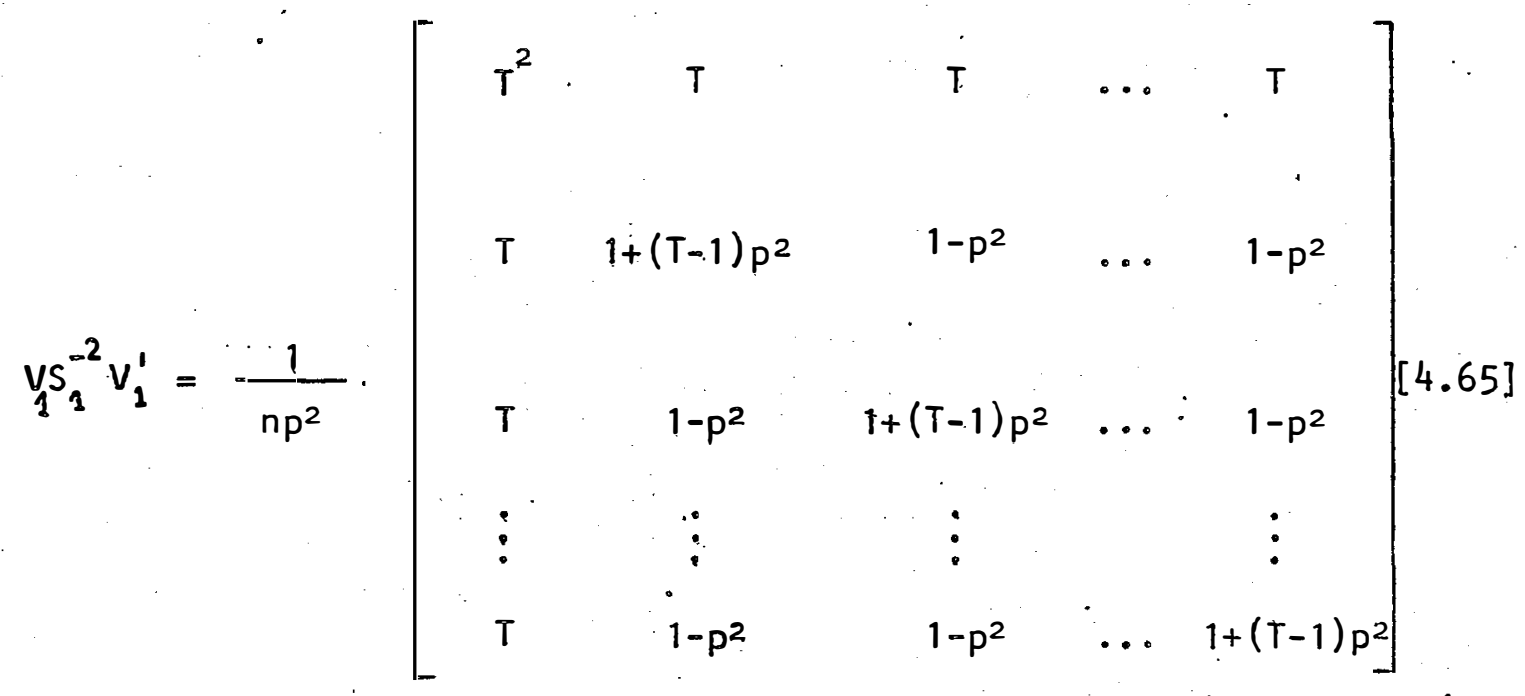

e a região de confiança ao nível 1-a para a coleção de m fun çöes paramētricas estimäveis $B^{\prime} \theta$ linearmente independente, é dada, segundo [4.26]

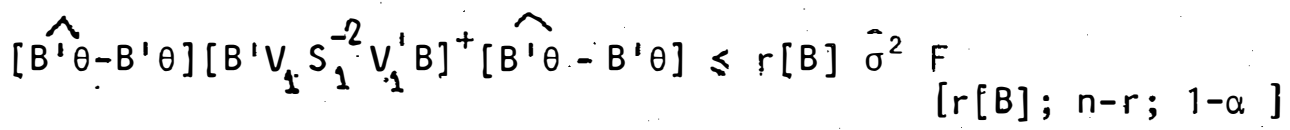

4.7. UM EXEMPLO NUMÉRICO

Face aos objetivos deste estudo, julgou-se oportuno apresentar um exemplo numérico para ilustrar o processo.

Seja, então, o conjunto de dados que constam do quadro 2 . 
Quadro 2 - Ensaio de competição de variedades e hidridos de milho na Estação Experimental de milho de Pira ju (SP), em t/ha.

Tratamentos

1ạ rep. 2ạ rep. 3ạ rep. 4ạ rep. 5ạ rep.

\begin{tabular}{llllll}
\hline Armour & 1.9 & 2,3 & 2.0 & 2.5 & 1.8 \\
Az.teca & 2,2 & 2,6 & 1.9 & 2.4 & 2.3 \\
H 6099 & 2.8 & 2.7 & 3.0 & 2.9 & 3.1 \\
H 3025 & 2.6 & 2.6 & 2.8 & 2.4 & 2.7 \\
IAC $40 / 65$ & 2.9 & 3.1 & 3.3 & 3.4 & 3.0 \\
IAC $65 / 70$ & 3.8 & 3.9 & 3.7 & 3.6 & 4.0 \\
\hline
\end{tabular}


.104.

A matriz do delineamento do presente estudo é

dada por:

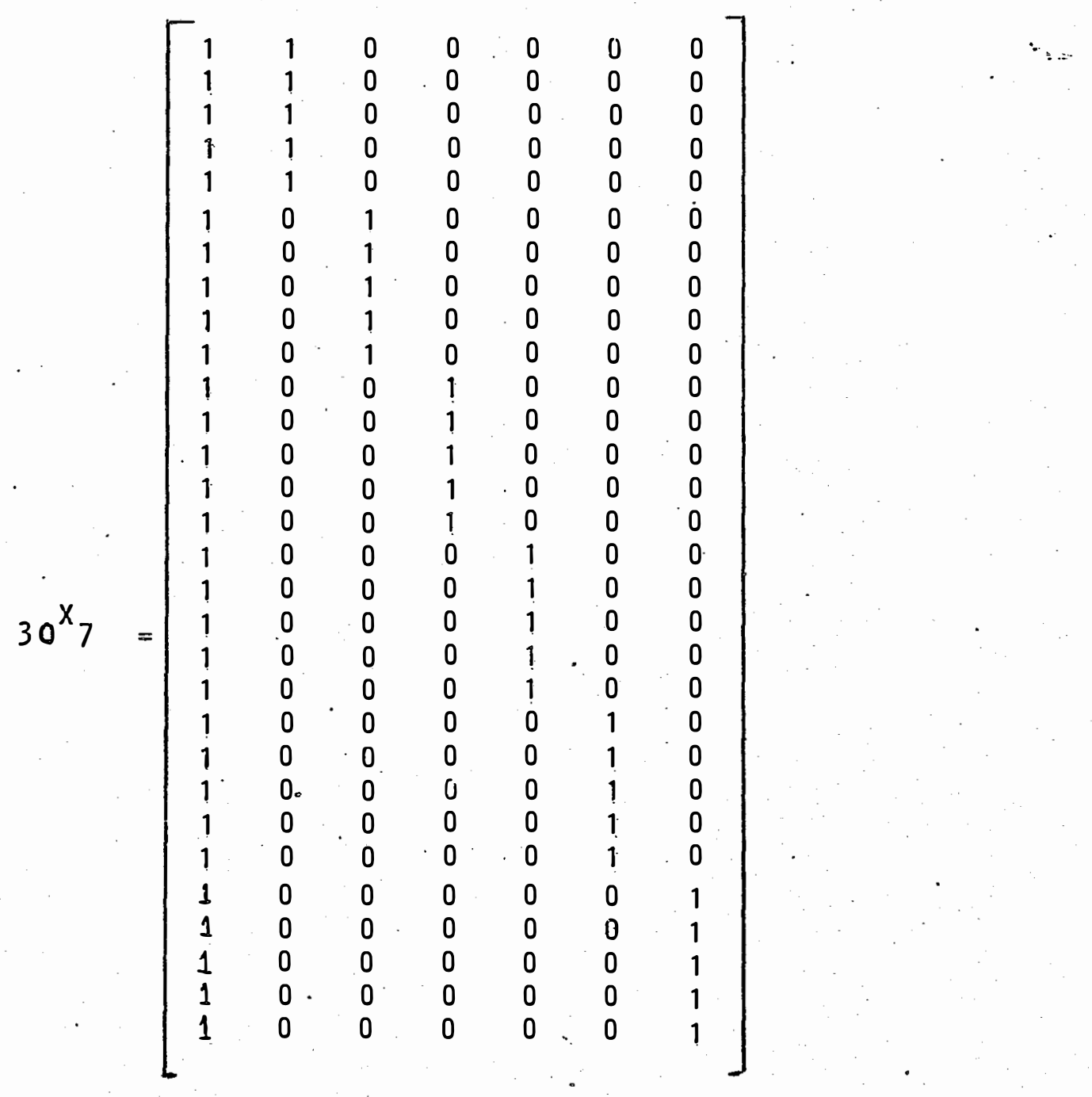


No exemplo tem-se

$$
N=30 \quad T=6 \quad R=5
$$

A D.V.S. da matriz $30^{X}, 7$, segundo o processo ASSIZ CARVALHO/IEMMA; : é dada por:

$$
\begin{aligned}
& U_{1}=\frac{1}{\sqrt{5}}\left(H_{(6)}^{1} \times 5^{E_{1}}\right) . \\
& S_{1}=\left[\begin{array}{cccccc}
\sqrt{(6+1) 5} & \phi & \phi & \phi & \phi & \phi \\
\phi & \sqrt{5} & \phi & \phi & \phi & \phi \\
\phi & \phi & \sqrt{5} & \phi & \phi & \phi \\
\phi & \phi & \phi & \sqrt{5} & \phi & \phi \\
\phi & \phi & \phi & \phi & \sqrt{5} & \phi \\
\phi & \phi & \phi & \phi & \phi & \sqrt{5}
\end{array}\right]
\end{aligned}
$$

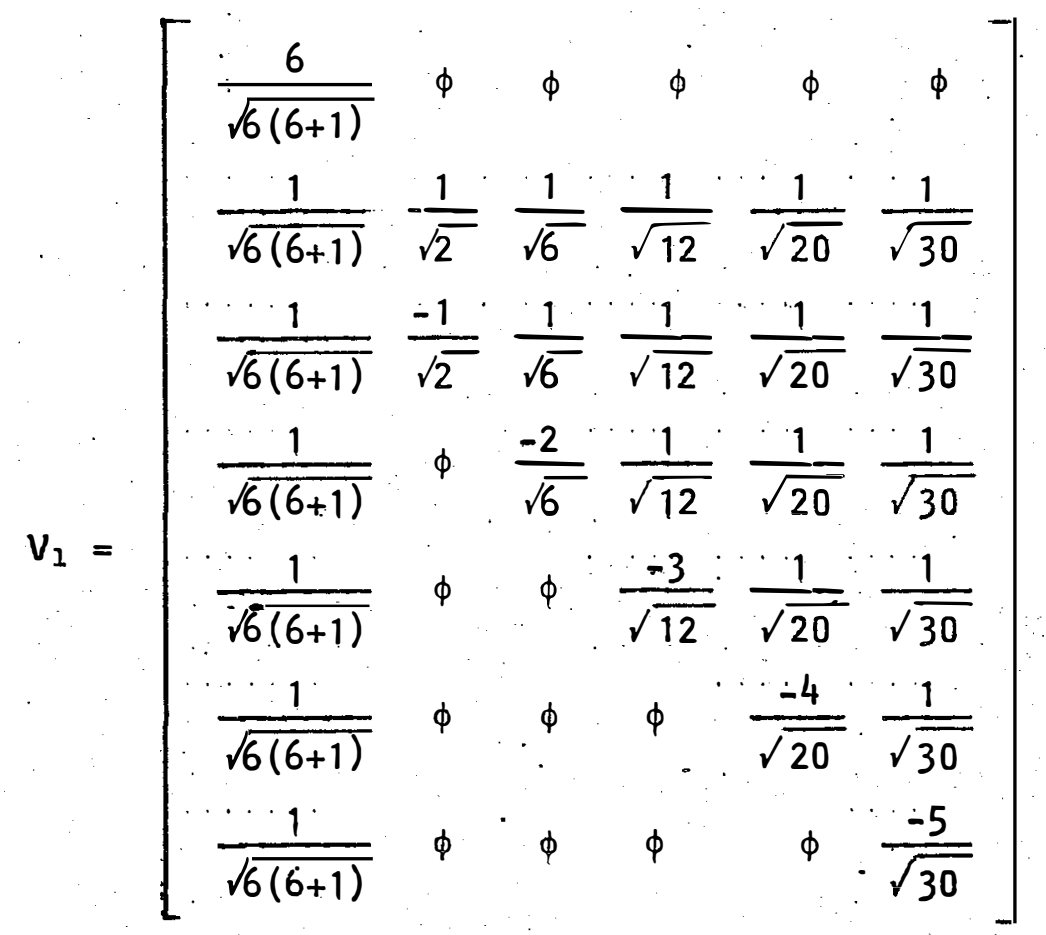




$$
\text { os projetores ortogonais em } C(x) \text { e } C\left(x^{\prime}\right) \text { são }
$$

dados por:

$$
\begin{aligned}
& P_{U_{1}}=\frac{1}{5} \quad I_{6} \otimes{ }_{5} E_{5} \\
& P_{V_{1}}=\frac{1}{6+1}\left[\begin{array}{rrrrrrr}
6 & 1 & 1 & 1 & 1 & 1 & -1 \\
1 & 6 & -1 & -1 & -1 & -1 & -1 \\
1 & -1 & 6 & -1 & -1 & -1 & -1 \\
1 & -1 & -1 & 6 & -1 & -1 & -1 \\
1 & -1 & -1 & -1 & 6 & -1 & -1 \\
1 & -1 & -1 & -1 & -1 & 6 & -1 \\
1 & -1 & -1 & -1 & -1 & -1 & 6
\end{array}\right]
\end{aligned}
$$

Segundo $[4.51], \theta^{0}$ é dado por:

$$
\theta^{0}=\left[\begin{array}{cc}
\bar{y}_{0} \\
\bar{y}_{1} \cdot & -\bar{y} \cdot . \\
\bar{y}_{2} \cdot & -\bar{y} \cdot . \\
\bar{y}_{3} \cdot & -\bar{y}_{\cdot} \\
\bar{y}_{4} \cdot & -\bar{y}_{\cdot} \\
\bar{y}_{5} \cdot & -\bar{y} \cdot \cdot \\
\bar{y}_{6} \cdot & -\bar{y} \cdot
\end{array}\right]=\left[\begin{array}{c}
2,8066 \\
2,1000-2,8066 \\
2,2800-2,8066 \\
2,9000-2,8066 \\
2,6200-2,8066 \\
3,1400-2,8066 \\
3,8000-2,8066
\end{array}\right]=\left[\begin{array}{c}
2,8066 \\
-0,7066 \\
-0,5266 \\
0,0934 \\
-0,1866 \\
0,3334 \\
0,9934
\end{array}\right]
$$

e segundo [4.52] a aproximação de mínimos quadrados para y é dada por:

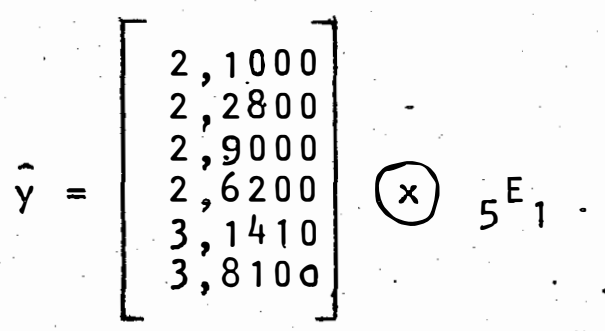




\section{Análise de Variància}

$$
\begin{aligned}
& y^{\prime} P_{U_{11}} y=\frac{\ldots\left[\Sigma \cdot y_{i j}\right]^{2}}{30}=236.3213 \\
& y^{\prime}\left(P_{U_{1}}-P_{U_{11}}\right) y=\frac{1}{5} \sum_{i=1}^{6} y_{i 0}^{2}-236.3213=9.5907 \\
& y^{\prime}\left(1-P_{U_{1}}\right) y=y^{\prime} y-y^{\prime} P_{U_{1}} y=246.98-245.9120=1.0680
\end{aligned}
$$

\begin{tabular}{|c|c|c|c|c|}
\hline CV & GL & SQ & $\mathrm{QM}$ & $F$ \\
\hline$\theta_{1}$ & 1 & . $\quad 236,3213$ & & \\
\hline$\theta_{2} / \theta_{1}$ & 5 & 9,5907 & 1.9181 & $43,10 * *$ \\
\hline Resíduo & 24 & 1,0680 & 0.0445 & \\
\hline Total 1 & 30 & 10.6587 & & \\
\hline
\end{tabular}

Quadro 3. Teste de $H_{0}: t_{1}=t_{2} \ldots=t_{6}$

Estimação por intervalo e por região

Testes sobre respostas

Nesse exemplo, são estimäveis as funções $\lambda ! \theta$ tais que $\lambda^{\prime} V_{1} V_{I}^{\prime}=\lambda^{\prime}$, ou se $j a$ : 


$$
\lambda \cdot \frac{1}{7}\left[\begin{array}{rrrrrrr}
6 & 1 & 1 & 1 & 1 & 1 & 1 \\
1 & 6 & -1 & -1 & -1 & -1 & -1 \\
1 & -1 & 6 & -1 & -1 & -1 & -1 \\
1 & -1 & -1 & 6 & -1 & -1 & -1 \\
1 & -1 & -1 & -1 & 6 & -1 & -1 \\
1 & -1 & -1 & -1 & -1 & 6 & -1 \\
1 & -1 & -1 & -1 & -1 & -1 & 6
\end{array}\right]=\lambda^{\prime} \text {; Proposição } 4
$$

Temrse, então, por exemplo, que

$$
\begin{aligned}
& \lambda^{\prime} \hat{\theta}=\bar{t}_{1}-\bar{t}_{6} ; \quad \lambda^{\prime}=\left(\begin{array}{lllllll}
0 & 1 & 0 & 0 & 0 & 0 & -1
\end{array}\right) \\
& \lambda^{\prime} P_{V_{1}}=\frac{1}{7}\left(\begin{array}{lllllll}
0 & 7 & 0 & 0 & 0 & 0 & -7
\end{array}\right)=\left(\begin{array}{ccccccc}
0 & 1 & 0 & 0 & 0 & 0 & -1
\end{array}\right)=\lambda^{\prime}
\end{aligned}
$$

è estimável, e de acordo com 4.2.6., o BLUE de $\lambda^{\prime} \theta$ é dado por

$$
\begin{aligned}
& \hat{\lambda^{\prime} \theta}=\lambda^{\prime} \theta^{0}=t_{1}^{0}-t_{6}^{0}=\bar{y}_{1}-\bar{y}_{.}-\left(\bar{y}_{6} \cdot-\bar{y}_{.}\right)=\bar{y}_{1} \cdot-\bar{y}_{6} \cdot=-1 \cdot 7 . \\
& v\left(\lambda^{\prime} \theta\right)=v\left(\lambda^{\prime} \theta^{0}\right)=\lambda^{\prime} v_{1} s_{1}^{-2} v_{1}^{1} \lambda
\end{aligned}
$$$$
V\left(\lambda^{\prime} \theta^{0}\right)=\lambda^{\prime} \frac{1}{30 \times 49}\left[\begin{array}{ccccccc}
49 & 6 & 6 & 6 & 6 & 6 & -48 \\
6 & 246 & -48 & -48 & -48 & -48 & -48 \\
6 & -48 & 246 & -48 & -48 & -48 & -48 \\
6 & -48 & -48 & 246 & -48 & -48 & -48 \\
6 & -48 & -48 & -48 & 246 & -48 & -48 \\
6 & -48 & -48 & -48 & -48 & 246 & -48 \\
6 & -48 & -48 & -48 & -48 & -48 & 246
\end{array}\right] \lambda \sigma^{2}
$$ 


$$
\text { Para } \lambda_{1}^{1}\left(\theta^{0}\right)=t_{1}^{0}-t_{6}^{0} \text {, tem-se que }
$$

$\lambda_{1}^{\prime}=\left(\begin{array}{lllllll}0 & 1 & 0 & 0 & 0 & 0 & -1\end{array}\right), \bar{e}$ de acordo com [4.66], obtēm-se:

$$
V\left(\lambda^{\prime} \theta^{0}\right)=0.4 \sigma^{2}
$$

Analogamente, para $\lambda_{2}^{1} \theta^{0}=t_{1}^{0}+t_{2}^{0}-2 t_{6}^{0}$, tem-se que.

$\lambda_{2}^{\prime}=\left(\begin{array}{lllllll}0 & 1 & 1 & 0 & 0 & 0 & -2\end{array}\right)$, e de acordo com [4.66] obtêm-se que:

$$
V\left(\lambda \cdot \theta^{0}\right)=1.2 \sigma^{2}
$$

Sabe-se, de [4.22], que uma estimativa por in tervalo ao nível de confiança $1-\alpha$ para $\lambda^{\prime} \theta$ estimävel, pode ser obtida de

$$
\text { I.C. }[\lambda ! \theta]_{1-\alpha}=\widehat{\lambda^{\prime} \theta \pm t} \underset{[\alpha / 2 ; n-T]}{\hat{\sigma}} \hat{\sqrt{V_{1} S_{1}^{-2} V_{I}^{\prime}}}
$$

assim sendo, para $\bar{\sigma}^{2}=0.0445$ obtêm-se:

$$
\begin{aligned}
& \hat{v}\left(\widehat{\lambda \hat{1} \theta_{1}}\right)=\frac{12}{30} \bar{\sigma}^{2}=0.0178 \\
& \hat{v}\left(\widehat{\lambda+\theta_{2}}\right)=\frac{1764}{1470} \cdot \bar{\sigma}^{2}=0.0534
\end{aligned}
$$

portanto, de acordo com [4.64],

$$
\begin{aligned}
& \text { I.C.[ } \left.t_{1}-t_{6}\right]_{0,95}=1.7 \pm 2,064\{0,0178\}^{1 / 2} \\
& \therefore P\left\{-1.9754 \leqq t_{1}-t_{2} \leqq-1.4246\right\}=0.95
\end{aligned}
$$


.110 .

$$
\begin{aligned}
& \text { I.C. }\left[t_{1}+t_{2}-2 t_{6}\right]_{0.95}=3.22 \pm 2.064 \cdot\{0,0534\}^{1 / 2} \\
& \therefore P\left\{-3.6970 \leqq t_{1}+t_{2}-2 t_{6} \leqq-2.7430\right\}=0.95 \\
& \text { Testes de Hipóteses baseados em regiões de con } \\
& \text { confi. ança } \\
& \text { Seja a função estimävel } B^{\prime} \theta=\left[\begin{array}{c}
\lambda_{1}^{\prime} \\
\hdashline \lambda_{2}^{1}
\end{array}\right] \theta \\
& \begin{aligned}
\operatorname{tq} \lambda_{1}^{\prime} \theta & =t_{1}-t_{6} e \\
\lambda_{2}^{\prime} \theta & =t_{1}+t_{2}-2 t_{6} ;
\end{aligned} \\
& \text { - Uma estimativa por região para } B^{1} \theta \text {, ao nível } \\
& \text { de confiança conjunta } 1-\alpha \text { é dado em [4.65], por } \\
& \left(B^{\prime} \theta^{0}-B^{\prime} \theta\right)^{\prime}\left(B^{\prime} V_{1} S_{1}^{-2} V_{1}^{\prime} B\right)^{+}\left(B^{\prime} \theta^{0}-B^{\prime} \theta\right) \leqq m \hat{\sigma}^{2} \underset{[n, n-r ; 1-\alpha]}{F} .
\end{aligned}
$$

Aqui $m=$ duas funções, $F_{0,05}=3.40 \mathrm{e}^{2}=0.0445 \ldots$

$$
\text { Então, para } \alpha=0.05
$$$$
\left[-3.22-\left(t_{1}+t_{2}-2 t_{6}\right) ;-1.7-\left(t_{2}-t_{6}\right)\right]\left[\begin{array}{ll}
\frac{1764}{1470} & \phi \\
\phi & \frac{588}{1470}
\end{array}\right]^{-1}\left[\begin{array}{l}
-3.22\left(t_{1}+t_{2}-2 t_{6}\right) \\
-1,7-\left(t_{1}-t_{6}\right)
\end{array}\right] \leq 2 \times 0,0445 \times 3,40
$$$$
\frac{\left[-3.22-\left(t_{1}+t_{2}-2 t_{6}\right)\right]^{2}}{\frac{1764}{1470} \times 0,0445 \times 2 \times 3.40}+\frac{\left[-1.7-\left(t_{1}-t_{2}\right]^{2}\right.}{\frac{588}{1470} \times 0,0445 \times 3,40 \times 2}=1
$$ 
$\frac{\left[-3.22-\left(t_{1}+t_{2}-2 t_{6}\right]^{2}\right.}{0,36312}+\frac{\left[-1.7-\left(t_{1}-t_{2}\right)\right]}{0,12104} \leq 1$

Tem-se, então, uma elipse $(m=2)$ de confiança para $B^{\prime} \theta^{0}$ com centro em $(-3.22 ;-1.7)$ e que não contém o ponto $(0,0)$. Graficamente, temos:

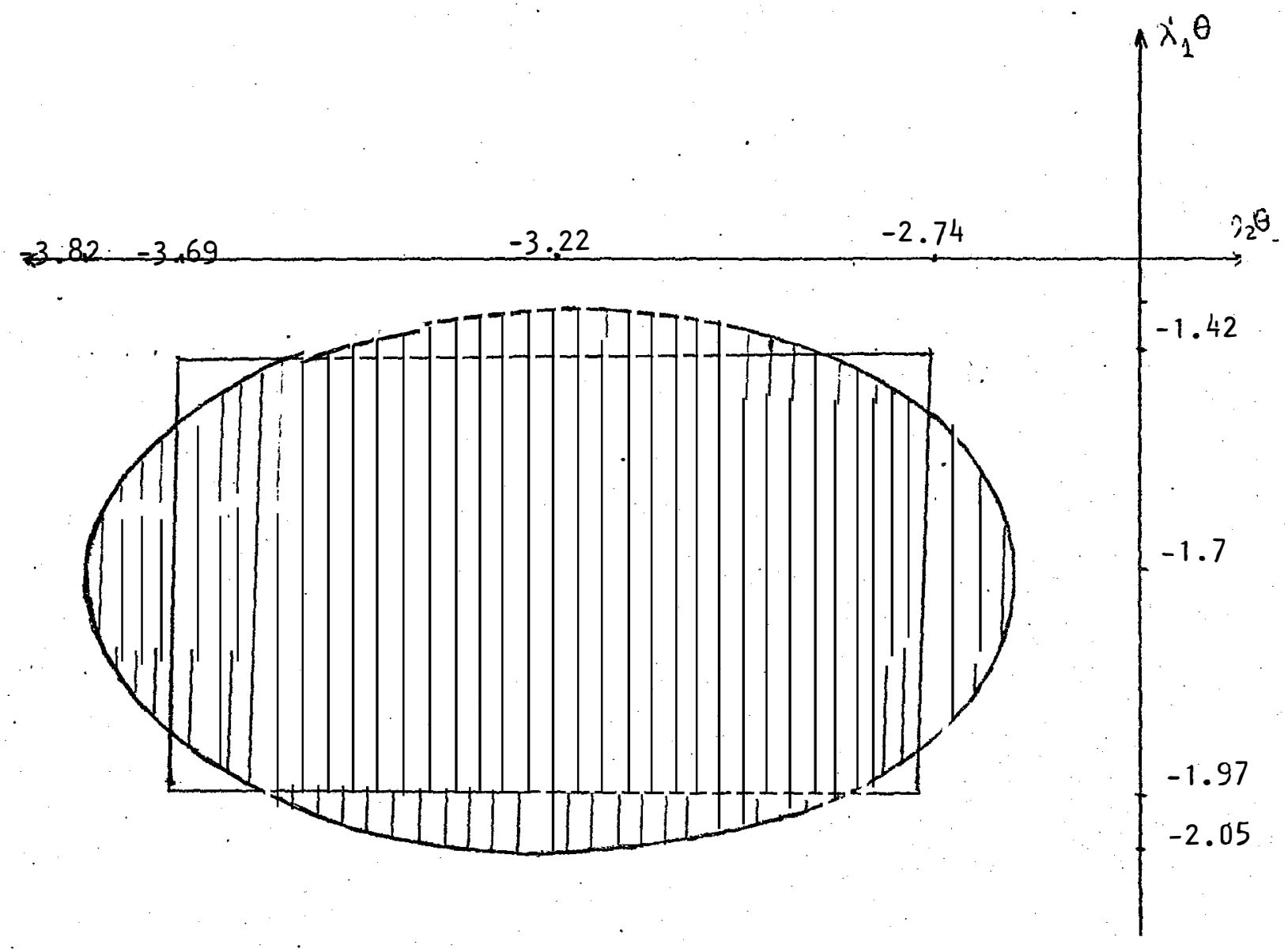

Figura 1 - Estimativa por região para $B^{\prime} \theta$ ao nível de confiança 0,95 . 
.112 .

\section{CONCLUSÕES}

Face aos resultados obtidos, conclui-se que o estudo do modelo linear de Gauss-Markov através do enfoque da D.V.S., possibilitou de modo simples e objetivo:

5.1. Obter a melhor solução aproximada (Solução de Norma Mínimol pára o sistema de equações normais, dada por $\theta^{\circ}=V_{1} S_{1}^{-1} U U_{1}$, sem utilizar explicitamente a inversa de Moore Penrose.

5.2. Demonstrar que $V_{1} S_{1}^{-1} U_{1}^{\prime}$ é a inversa generalizada de Moore-Penrose de $X$.

5.3. Demonstrar que. a função $\lambda^{\prime} \theta$ e estimável no modelo experimental inteiramente casualizado com $T$ tratamentos e $R$ repetições se e só se $\sum_{i=1}^{p} \lambda_{i}=2 \lambda_{1}$.

5.4. Criar um algoritmo "Algoritmo Assiz Carvalho/lemma" que decompõe pelo valor singular qualquer matriz de planejamento $x$, desde que $x x^{i}$ seja $k^{2}$ - uniforme, sem necessidade de recursos computacionais.

Nesse contexto, permite-se entender que o enfoque através da D.V.S. revelou-se. extremamente rico em informações do ponto de vista das deduções teóricas e das exem plificações, e consequentemente como recurso didático. 


\section{REFERÊNCIAS BIBLIOGRĀFICAS}

ACTON, F.S. Numerical Methods that Work. Harper $\varepsilon$ Row, Publishers. New York, 1.970. $215 \mathrm{p}$.

BAYLEY, D.W. E JACOB, H.G. Linear Algebra. Houghton Mifflin Company. Boston, 1971. $280 \mathrm{p}$.

CARVALHO, J.F. Modelos Lineares e sua Avaliação. São Carlos, 1982. 95 p. (Livre Docência - Universidade de São Paulo)..

COHEN, A.M. Numerical Analysis. McGraw Hill Inc. New York, 1973. $\quad 341 \mathrm{p}$

DWIVEDI, T.D. A Method to Compute the Rank Factors of a a Matriz. Sankhyã, $37($ B. 4): 463-64, 1975 .

GRAYBILL, F.A. An introduction to Linear Statistical Models. Vol. 1. McGraw Hill. $463 \mathrm{p}$.

GRAYBILL, F.A. Introduction to Matrices with Aplications in Statistics. Wadworth, 1969. 372 p.

GOMES, F.P. Curso de Estatística Experimental. Nobel, 1967. $430 \mathrm{P}$.

HENRICI; P. On the Speed of Convergence of Cyclic and 
Quasicyclic Jacobi Methods for Computing Eigen Values of Hermitian Matrices. J. Soc. Industr. Appl. Math., 6:144$162,1958$.

IEMMA, A.F. Valores Singulares de Matrizes Uniformes. Ciên cia e Natura, 4:21-26, 1982.

IEMMA, A.F. Modelos Lineares para Experimentos com Medidas Repetidas e Covariância Uniforme: Um Enfoque Através das Matrizes de Helmert. Pi.racicaba, 1985. 155 p. (Livre-Do cência - Escola Superior de Agricultura "Luiz de Queiroz" (USP)

IEMMA, A.F. Modelos Lineares: Uma Introdução para Profissio nais da Pesquisa Agropecuāria. In: 2\% Simpōsio de Estațística Apliccada à Experimentação Agronômica; Londrina, PR, 1987. $263 \mathrm{P} \cdot \quad$ (c)

IEMMA, A.F. Usando Matrizes de Helmert e Matrizes Uniformes como Recurso Didático. In: I Simpósio de Métodos Quantitativos e $\mid 1$ Encontro de Econometria da Região Sul, Santa Maria, RS, Anais, $1987^{\circ}$ (a).

IEMMA, A.F. Inversa Genralizada de Moore-Penrose nos Delineamentos com Restrição na Casualização. In: $10^{\text {a }}$ Reunião Regional da SBMAC. São Carlos, SP. Anais, 1987 (b).

JOHN, P.W.M. Statistical. Design and Analysis of Experiments. Macmillan, 1971. $356 \mathrm{p}$. 
LIMA FILHo, E.C. Planejamențo de Observações Adicionais em Modelos Lineares: A Decomposição em Valores Singulares da Matriz de Planejamento e sua Utilização. São Carlos, 1981. 60 p. (Livre Docência - Universidade de São Pau10)

MANDEL, J. Use of Singular Decomposition in Regression Analysis. The American Statistician, 36(1):15-24; 1982 .

MURDOCH, D.C. Algebra Linear. Livros Técnicos e Cientificos: Rio de Janeiro, 1970. 310 p.

RAO, C.R. On the Linear Combination of Observations and the General Theory of Least Squares. Sankhya, The Ind. J. of Stat., $7(3): 237-256,1945$.

RAO, C.R. Linear Statistical Inference and its Applications. Wiley, 1965. 552 p.

SEARLE, S.R. Linear Models. Wiley, 197.1. 532 p.

SEARLE, S.R. Matrix Algebra Useful for Statistics. John Wiley E Sons.. New York, 1982. 438 p.

SEARLE, S.R. Restriction and.Generalized Inverses in Linear Models. The American Statistician, 38:53-54, 1984.

SEBER, G.A.F. Linear Regression Analysis. Wiley, 1977. 466 $\mathrm{p}$.

STEINBERG, D.I. Computational Matrix Algebra. McGraw-Hill Inc. New York, 1974. 280 p. 
.116 .

WILKINSON, J.H. The Algebraic Eigenvalue Problem. Claredon Press. 0xford. 1964. 662 p. 